\title{
Comportamento assintótico de problemas de difusão não locais e semilineares do tipo Neumann
}

Patrícia Neves de Araújo

\author{
DisSERTAÇÃO APRESENTADA \\ AO \\ Instituto De MatemáticA E EstatísticA \\ DA \\ Universidade De SÃo PAulo \\ PARA \\ OBTENÇÃO DO TÍTULO \\ $\mathrm{DE}$ \\ Mestre em CiÊnCIAS \\ Programa: Matemática Aplicada \\ Orientador: Prof. Dr. Marcone Corrêa Pereira
}

Durante o desenvolvimento deste trabalho a autora recebeu auxílio financeiro da CAPES

São Paulo, junho de 2019 



\title{
Comportamento assintótico \\ de problemas de difusão \\ não locais e semilineares \\ do tipo Neumann
}

\begin{abstract}
Esta versão da dissertação contém as correções e alterações sugeridas pela Comissão Julgadora durante a defesa da versão original do trabalho, realizada em 02/07/2019. Uma cópia da versão original está disponível no Instituto de Matemática e Estatística da Universidade de São Paulo.
\end{abstract}

Comissão Julgadora:

- Prof. Dr. Marcone Corrêa Pereira (orientador) - IME-USP

- Prof. Dr. Antonio Luiz Pereira - IME-USP

- Prof ${ }^{\mathrm{a}}$. Dra ${ }^{\mathrm{a}}$. Silvia Sastre Gomez - UFPE 


\section{Agradecimentos}

Agradeço primeiramente a Deus por me permitir ultrapassar os obstáculos que sempre surgem no caminho e chegar até aqui.

Agradeço aos meus pais, Iva e Monteiro, por me apoiarem sempre e incondicionalmente. Obrigada por todo amor e paciência, nada disso seria possível se não fosse por vocês. Agradeço à minha irmã Paula por sempre estar disposta a me ajudar e por ter tanta paciência para lidar comigo. Cada parte desse trabalho tem um pouco de vocês e eu os amo imensamente.

Gostaria também de agradecer ao Rafael, meu noivo, por estar comigo em todos os momentos e apoiar os meus sonhos. Te amo infinitamente.

Agradeço ao meu orientador, Prof. Dr. Marcone Corrêa Pereira, por toda a gentileza e disponibilidade em me orientar. Muito obrigada por tornar possível a realização deste trabalho.

Agradeço aos colegas e professores que contribuíram para o desenvolvimento desta pesquisa, tanto em discussões em aulas, quanto em seminários e outros momentos.

Agradeço ainda a todos os que me apoiaram e, de perto ou de longe, acompanharam esta jornada.

Muito obrigada! 


\section{Resumo}

ARAÚJO, P. N. de Comportamento assintótico de problemas de difusão não locais e semilineares do tipo Neumann. 2019. 70 f. Dissertação (Mestrado) - Instituto de Matemática e Estatística, Universidade de São Paulo, São Paulo, 2019.

Neste trabalho abordamos dois exemplos de equações de difusão não locais do tipo Neumann: o problema linear homogêneo e um semilinear com termo de reação representado pela função $f(u)=|u|^{p-1} u$. Em ambos os casos, apresentamos condições de existência e unicidade de soluções e analisamos seu comportamento em relação ao tempo. Estudamos uma discretização para o problema linear e a utilizamos para realizar simulações numéricas nas quais podemos verificar algumas das propriedades demonstradas. Também simulamos o problema semilinear observando o comportamento de suas soluções mesmo em casos em que as hipóteses dos teoremas apresentados não são todas satisfeitas.

Palavras-chave: equações não locais, problemas do tipo Neumann, comportamento assintótico, blow-up. 


\section{Abstract}

\section{ARAÚJO, P. N. de Asymptotic behavior of nonlocal and semilinear diffusion problems}

of Neumann type. 2019. 70 f. Dissertação (Mestrado) - Instituto de Matemática e Estatística, Universidade de São Paulo, São Paulo, 2019.

In this work we approach two examples of nonlocal diffusion equations of Neumann type: the homogeneous linear problem and a semilinear with a reaction term represented by the function $f(u)=|u|^{p-1} u$. In both cases, we present conditions of existence and uniqueness of solutions and we analyze their behavior with respect to time. We study a discretization to the linear problem and use it to perform numerical experiments in order to illustrate some of the demonstrated properties. We also simulate the semilinear problem observing the behavior of its solutions even in cases where the hypothesis of the presented theorems are not all satisfied.

Keywords: nonlocal equations, problems of Neumann type, asymptotic behavior, blow-up. 


\section{Sumário}

$\begin{array}{ll}\text { Lista de Figuras } & \text { ix }\end{array}$

$\begin{array}{ll}\text { Introdução } & 1\end{array}$

1 Contextualização e descrição do problema $\quad 3$

2 Problema de difusão não local do tipo Neumann homogêneo $\quad 9$

2.1 Existência e unicidade de soluções . . . . . . . . . . . . . . . . . . 9

2.2 Comportamento assintótico das soluções . . . . . . . . . . . . . . . . . . . . 12

2.3 Subsoluções e supersoluções . . . . . . . . . . . . . . . . . . . . . 19

3 Problema com condições de Neumann e um termo de reação 21

3.1 Existência e unicidade de soluções . . . . . . . . . . . . . . . . . . . . 21

3.2 Condições e taxas de explosão de soluções . . . . . . . . . . . . . . . . . 27

4 Tratamento numérico de problemas não locais $\quad 41$

4.1 Discretização do operador linear . . . . . . . . . . . . . . . . . . . . . . . . 41

4.1 .1 Esquema semidiscreto . . . . . . . . . . . . . . . . . . . 42

4.1 .2 Esquema totalmente discreto f . . . . . . . . . . . . . . 46

4.2 Simulações numéricas . . . . . . . . . . . . . . . . . . . 48

4.2 .1 Problema linear . . . . . . . . . . . . . . . . . . . . . . . . . 49

4.2 .2 Problema com termo de reação . . . . . . . . . . . . . . . . . 52

$\begin{array}{ll}\text { Conclusões } & 66\end{array}$

$\begin{array}{ll}\text { Referências Bibliográficas } & 69\end{array}$ 


\section{Lista de Figuras}

4.1 Gráfico da solução do problema 1 para determinados valores de $t$. . . . . . . . . . 50

4.2 Gráfico da solução do problema $1 . \ldots \ldots \ldots$. . . . . . . . . . 5 . 50

4.3 Gráfico da solução do problema 2 para determinados valores de $t$. . . . . . . . . . . 51

4.4 Gráfico da solução do problema $2 \ldots \ldots \ldots$. . . . . . . . . . . . 51

4.5 Gráfico da solução do problema 3 para determinados valores de $t$. . . . . . . . . . . 52

4.6 Gráfico da solução do problema $3 . \ldots \ldots \ldots$. . . . . . . . . . . . . 52

4.7 Gráfico da solução do problema 4 para determinados valores de $t$. . . . . . . . . . 53

4.8 Gráfico da solução do problema $4 . \ldots \ldots \ldots$. . . . . . . . . . . . 54

4.9 Gráfico da solução do problema 5 para determinados valores de $t$. . . . . . . . . . 55

4.10 Gráfico da solução do problema $5 . \quad \ldots \ldots \ldots$. . . . . . . . . . . 55

4.11 Gráfico da solução do problema 6 para determinados valores de $t$. . . . . . . . . . 56

4.12 Gráfico da solução do problema $6 . \ldots \ldots \ldots \ldots$. . . . . . . . . . 57

4.13 Gráfico da solução do problema 7 para determinados valores de $t$. . . . . . . . . . 58

4.14 Gráfico da solução do problema $7 . \ldots \ldots \ldots \ldots$

4.15 Gráfico da solução do problema 8 para determinados valores de $t$. . . . . . . . . . . 59

4.16 Gráfico da solução do problema $8 . \ldots \ldots$. . . . . . . . . . . . . 60

4.17 Gráfico da solução do problema 9 para determinados valores de $t$. . . . . . . . . . . . 61

4.18 Gráfico da solução do problema $9 . \ldots \ldots 1$

4.19 Gráfico da solução do problema 10 para $t=5,754 \cdot 10^{-1}$ no intervalo $[-0,08,0,08]$. . 62

4.20 Gráfico da solução do problema 10 . . . . . . . . . . . . . . . 63

4.21 Gráfico da solução do problema 11 para determinados valores de $t$. . . . . . . . . . . 64

4.22 Gráfico da solução do problema 11. . . . . . . . . . . . . . . . . . . . . 64

4.23 Gráfico da solução do problema 12 para determinados valores de $t$. . . . . . . . . . . 65

4.24 Gráfico da solução do problema $12 \ldots \ldots$. . . . . . . . . . . . . 65 


\section{Introdução}

Neste trabalho, estudaremos uma classe de equações não locais com condições de Neumann homogêneas amplamente utilizadas na modelagem de fenômenos que envolvem difusão. Para tanto, consideramos ao longo de todo o trabalho $\Omega$ um aberto limitado em $\mathbb{R}^{N}$ e $J \in C^{1}\left(\mathbb{R}^{N}, \mathbb{R}\right)$ uma função não negativa, radial, contínua, com $J(0)>0$ e tal que

$$
\int_{\mathbb{R}^{N}} J(x) d x=1 .
$$

Note que tais propriedades caracterizam $J$ como uma função de densidade de probabilidade.

O problema que trataremos inicialmente é

$$
\begin{cases}u_{t}(x, t)=\int_{\Omega} J(x-y)(u(y, t)-u(x, t)) d y, & x \in \Omega, t>0, \\ u(x, 0)=u_{0}(x), & x \in \Omega .\end{cases}
$$

Esta formulação é conhecida como problema de difusão não local do tipo Neumann homogênea e é amplamente utilizada em diversas áreas, como redução de ruído em imagens [GO07], peridinâmica [TD13] e sistemas de partículas [OS17].

O outro problema a ser estudado é

$$
\begin{cases}u_{t}(x, t)=\int_{\Omega} J(x-y)(u(y, t)-u(x, t)) d y+|u|^{p-1} u(x, t), & x \in \Omega, t>0 \\ u(x, 0)=u_{0}(x), & x \in \Omega,\end{cases}
$$

que pode ser entendido como um problema de difusão não local com condições de Neumann e um termo de reação não linear representado pela função monótona $|u|^{p-1} u$. Aqui assumimos $p>0$.

O objetivo deste trabalho é estudar propriedades referentes aos problemas mencionados, como existência, unicidade e comportamento assintótico das soluções no tempo. Além disso, apresentaremos uma discretização que será utilizada para obter simulações que nos permitam observar o comportamento das soluções para domínios $\Omega$ unidimensionais.

O Capítulo 1 será dedicado a uma breve apresentação das equações não locais (1) e (2), suas motivações e relações com os problemas de difusão locais, com base nos estudos realizados por Fife [Fif03] e Sastre [Góm14]. No Capítulo 2, apresentaremos um estudo mais rigoroso do problema (1), cuja referência principal é o livro de Andreu-Vaillo et al. [AVt10]. Nesse Capítulo, verificaremos condições de existência e unicidade de soluções e o comportamento assintótico das mesmas, além de um princípio de comparação. No Capítulo 3, estudaremos as propriedades do problema (2), como condições de existência local e global, unicidade de soluções e taxa de explosão para os casos que apresentam blow-up com base em [PLR09] e utilizando [GR01] como material de apoio.

No Capítulo 4 apresentamos um tratamento numérico para as equações (1) e (2). Para isto, seguiremos o artigo [PLR11] para estudo de uma discretização. Além disso, apresentaremos simulações numéricas sobre as quais poderemos verificar algumas propriedades demonstradas e conjecturar algumas outras com base na análise dos gráficos obtidos. 


\section{Capítulo 1}

\section{Contextualização e descrição do problema}

Os problemas não locais vêm sendo amplamente estudados devido à sua aplicação em diversas áreas do conhecimento. Em Biologia, por exemplo, os problemas estão relacionados à dinâmica de populações, sendo utilizados para analisar seu comportamento mediante determinadas condições. Nessa direção veja Carillo e Fife [CF05] onde uma formulação não local foi utilizada para analisar o comportamento de uma espécie sob específicas leis de movimento. Outro exemplo é a argumentação apresentada por Hutson et al. em [HMMV03], que também analisam o movimento de indivíduos em um habitat.

Outra área na qual os problemas não locais estão sendo utilizados é a computação. Em [Tt18] se analisa redes neurais não locais e em [G008] se estuda operadores não locais com aplicações em processamento de imagens. Em Física Estatística, o trabalho de Fournier e Laurençot [FL06] avalia a equação de coagulação de Smoluchowski em uma classe de núcleos homogêneos.

Além das aplicações em outras áreas, os operadores não locais estão sendo utilizados para estudar modelos matemáticos propostos por equações diferenciais. Neste sentido, destacamos o estudo da equação de Cahn-Allen não local apresentado em [BC02] e o artigo [SMD18], dedicado a apresentar algoritmos para resolução de modelos não locais na esfera (entre eles, a equação de Cahn-Allen).

Neste capítulo, veremos como podemos relacionar um operador não local ao operador de Laplace a fim de compreender os modelos de difusão não local.

Iniciaremos considerando uma população em um habitat $N$-dimensional cuja densidade na posição $x$ no instante $t$ é representada por uma função $u(x, t)$.

Para o caso $N=1$, temos a seguinte formulação, conforme apresentado por Sastre em [Góm14]. Seja $\Omega \subset \mathbb{R}$ um intervalo representando o habitat considerado. Dividindo-o em partes de tamanho $\Delta x$ fixo, obtemos uma malha unidimensional $\left\{x_{i}\right\},-M \leq i \leq M$, na qual analisaremos a densidade populacional. Discretizamos o tempo em partes de tamanho $\Delta t$. Seja $u(i, t)$ a densidade de população no intervalo $i$, isto é, no intervalo $\left[x_{i-1}, x_{i}\right]$ para $-M<i \leq M$, no tempo $t$. Assumimos primeiramente que a taxa na qual os indivíduos saem da posição $i$ para a posição $j$ é constante. Consideramos que o número de indivíduos saindo do intervalo $i$ para $j$ é proporcional à população no intervalo $i$, dada por $u(i, t) \Delta x$, ao tamanho do intervalo, que é $\Delta x$, e também ao intervalo de tempo durante o qual o trânsito de indivíduos está sendo analisado, isto é, $\Delta t$. Assim, se denotamos por $J(j, i)$ a constante de proporcionalidade temos que o número de indivíduos saindo da posição $i$ para a posição $j$ durante o intervalo $[t, t+\Delta t]$ é

$$
J(j, i) u(i, t) \Delta t(\Delta x)^{2} .
$$

Analogamente, o número de indivíduos que chegam em $i$, vindos de todas as posições, durante 
o mesmo intervalo, é

$$
\sum_{\substack{j=-M+1 \\ j \neq i}}^{M} J(i, j) u(j, t) \Delta t(\Delta x)^{2}
$$

Temos assim que a quantidade de indivíduos em $i$ no tempo $t+\Delta t$ é dada pela soma dos indivíduos que lá estavam com os que vieram de outras posições, menos os que saíram, isto é,

$$
u(i, t+\Delta t) \Delta x=u(i, t) \Delta x+\sum_{\substack{j=-M+1 \\ j \neq i}}^{M} J(i, j) u(j, t) \Delta t(\Delta x)^{2}-\sum_{\substack{j=-M+1 \\ j \neq i}}^{M} J(j, i) u(i, t)(\Delta x)^{2}(\Delta t),
$$

ou seja,

$$
u(i, t+\Delta t) \Delta x-u(i, t) \Delta x=\sum_{\substack{j=-M+1 \\ j \neq i}}^{M} J(i, j) u(j, t)(\Delta x)^{2} \Delta t-\sum_{\substack{j=-M+1 \\ j \neq i}}^{M} J(j, i) u(i, t)(\Delta x)^{2}(\Delta t) .
$$

Dividindo os dois lados da equação por $\Delta x$ e $\Delta t$, obtemos

$$
\frac{u(i, t+\Delta t)-u(i, t)}{\Delta t}=\sum_{\substack{j=-M+1 \\ j \neq i}}^{M} J(i, j) u(j, t) \Delta x-\sum_{\substack{j=-M+1 \\ j \neq i}}^{M} J(j, i) u(i, t) \Delta x .
$$

Tomando então os limites $\Delta t \rightarrow 0$ e $\Delta x \rightarrow 0$, obtemos

$$
u_{t}(x, t)=\int_{\Omega}(J(x, y) u(y, t)-J(y, x) u(x, t)) d y .
$$

Essa expressão nos fornece informações sobre a variação instantânea da densidade populacional em um ponto $x \in \Omega$. Aplicando o mesmo raciocínio para $N>1$, obteremos a mesma expressão. O problema (1.1) é chamado de problema de difusão não local por considerar o movimento de indivíduos não apenas na posição $x$, mas em todo o domínio.

Consideramos uma função $J: \mathbb{R}^{n} \rightarrow \mathbb{R}$ satisfazendo as seguintes condições

$$
\begin{gathered}
J \in C\left(\mathbb{R}^{N}, \mathbb{R}\right) \text { é não negativa, com } J(x)=J(-x) \text {, limitada }\left(\operatorname{com} K=\|J\|_{\infty}\right) \text { tal que } \\
\qquad J(0)>0 \text { e } \int_{\mathbb{R}^{N}} J(z) d z=1 .
\end{gathered}
$$

A hipótese (H) caracteriza $J$ como uma função de "densidade de probabilidade".

No estudo de difusão local, uma das condições de contorno mais comuns é a de Dirichlet. No caso, a difusão ocorre em $\mathbb{R}^{N}$, porém devemos definir o valor que ela assume fora do domínio $\Omega$. Assim, a função $u(x, t)$ satisfaz a expressão

$$
\begin{cases}u_{t}(x, t)=\int_{\mathbb{R}^{N}} J(x-y)(u(y, t)-u(x, t)) d y, & x \in \Omega, \quad t>0 \\ u(x, 0)=u_{0}(x), & x \in \Omega \\ u(x, t)=g(x), & x \notin \Omega .\end{cases}
$$

Esta é a versão não local do problema de difusão com condições de Dirichlet. Note que a "condição de contorno" é não homogênea se $g \neq 0$.

Caso estejamos modelando problemas nos quais não há entrada ou saída de indivíduos num determinado habitat $\Omega$ (ou seja, aos indivíduos se permite movimentação apenas em $\Omega$ ), temos que 
a difusão ocorre apenas em $\Omega$ e logo procuramos soluções para o problema

$$
\begin{cases}u_{t}(x, t)=\int_{\Omega} J(x-y)(u(y, t)-u(x, t)) d y, & x \in \Omega, \quad t>0, \\ u(x, 0)=u_{0}(x), & x \in \Omega .\end{cases}
$$

Este é o nosso objeto de interesse neste estudo, a versão não local do problema com condições de Neumann ditas homogêneas, como destacam Sastre [Góm14], Fife [Fif03], Andreu-Vaillo et al. [AVt10] e diversos outros autores.

Como visto, podemos utilizar formulações não locais para analisar problemas que são comumente modelados por equações locais. Veremos, a seguir, outra forma de relacionar estes dois tipos de problemas. O estudo proposto por Fife [Fif03] relaciona o problema semilinear com condições de Neumann homogêneas

$$
u_{t}=\Delta u-f(u)
$$

definido pelo operador Laplaciano $\Delta=\sum_{i=1}^{N} \frac{\partial^{2}}{\partial x_{i}^{2}}$ à sua versão não local

$$
u_{t}(x, t)=\int_{\Omega} J(x-y)(u(y, t)-u(x, t)) d y-f(u(x, t)) .
$$

Caracterizaremos primeiramente $-\Delta u$ como a derivada em $u$ da integral

$$
E_{0}(u)=\int_{\Omega} \frac{1}{2}|\nabla u|^{2} d x
$$

De fato, temos que

$$
\frac{d}{d t} E_{0}(u)=\int_{\Omega} \nabla u \nabla u_{t} d x=-\int_{\Omega} u_{t} \Delta u d x+\int_{\partial \Omega} u_{t} \frac{\partial u}{\partial \eta} d S,
$$

onde $\eta$ é o vetor normal a $\partial \Omega$ apontando para fora. Aqui utilizamos a identidade de Green.

Logo, com condições de contorno apropriadas (como $u_{t} \equiv 0$ ou $\frac{\partial u}{\partial \eta} \equiv 0$ em $\partial \Omega$ para todo $t \geq 0$ ) obtemos

$$
\frac{d}{d t} E_{0}(u)=-\int_{\Omega} u_{t} \Delta u d x=<u_{t},-\Delta u>
$$

em $L^{2}(\Omega)$ para toda função $u$ suave nessas condições. Aqui $<\cdot, \cdot>$ denota o produto interno em $L^{2}(\Omega)$. Assim, podemos escrever formalmente que

$$
\frac{\partial E_{0}}{\partial u}=-\Delta u
$$

Observe que $E_{0}(u) \geq 0$. Além disso, $E_{0}(u)=0 \Longleftrightarrow u$ é constante. Logo, a energia $E_{0}(u)$ mede o quanto a função $u$ está distante de ser constante. Uma forma alternativa de obter essa medida é

$$
E_{10}(u)=\int_{\Omega} \int_{\Omega} \frac{1}{4} J(x-y)(u(x)-u(y))^{2} d x d y,
$$

onde $J$ satisfaz as condições dadas pela hipótese $(\mathrm{H})$. Vemos que $E_{10}(u) \geq 0$ com $E_{10}=0 \Longleftrightarrow u$ é constante em $\Omega$. Mas $J$ é par e $(u(x)-u(y))^{2}=(u(x)-u(y)) u(x)-(u(x)-u(y)) u(y)$. Logo

$$
\begin{aligned}
E_{10}(u) & =\int_{\Omega} \int_{\Omega} \frac{1}{4} J(x-y)(u(x)-u(y)) u(x) d x d y-\int_{\Omega} \int_{\Omega} \frac{1}{4} J(x-y)(u(x)-u(y)) u(y) d x d y \\
& =\int_{\Omega} \int_{\Omega} \frac{1}{4} J(x-y)(u(x)-u(y)) u(x) d x d y+\int_{\Omega} \int_{\Omega} \frac{1}{4} J(y-x)(u(y)-u(x)) u(y) d y d x
\end{aligned}
$$


e, observando que as parcelas são iguais, obtemos que

$$
E_{10}(u)=\int_{\Omega} \int_{\Omega} \frac{1}{2} J(x-y)(u(x)-u(y)) u(x) d x d y .
$$

Logo

$$
\frac{d}{d t} E_{10}(u)=\frac{d}{d t} \int_{\Omega} \int_{\Omega} \frac{1}{2} J(x-y)(u(x)-u(y)) u(x) d x d y .
$$

Daí, temos

$$
\begin{aligned}
\frac{d}{d t} E_{10}(u) & =\frac{d}{d t} \int_{\Omega} \int_{\Omega} \frac{1}{2} J(x-y)(u(x)-u(y)) u(x) d x d y \\
& =\frac{1}{2} \int_{\Omega} \int_{\Omega} J(x-y)\left(\left(u_{t}(x)-u_{t}(y)\right) u(x)+(u(x)-u(y)) u_{t}(x)\right) d x d y \\
& =\int_{\Omega} \int_{\Omega} J(x-y) u(x) u_{t}(x) d x d y-\int_{\Omega} \int_{\Omega} \frac{1}{2} J(x-y) u_{t}(y) u(x) d x d y-\int_{\Omega} \int_{\Omega} \frac{1}{2} J(x-y) u_{t}(x) u(y) d x d y .
\end{aligned}
$$

Utilizando a simetria de $J$, temos que

$$
\int_{\Omega} \int_{\Omega} \frac{1}{2} J(x-y) u_{t}(y) u(x) d x d y=\int_{\Omega} \int_{\Omega} \frac{1}{2} J(x-y) u_{t}(x) u(y) d x d y,
$$

e portanto

$$
\begin{aligned}
\frac{d}{d t} E_{10}(u) & =\int_{\Omega} \int_{\Omega} J(x-y) u(x) u_{t}(x) d x d y-\int_{\Omega} \int_{\Omega} J(x-y) u_{t}(x) u(y) d x d y \\
& =\int_{\Omega} \int_{\Omega} J(x-y)(u(x)-u(y)) u_{t}(x) d x d y \\
& =\left\langle-\int_{\Omega} J(x-y)(u(y)-u(x)) d y, u_{t}(x)\right\rangle .
\end{aligned}
$$

Assim, se escrevemos $A(x)=\int_{\Omega} J(x-y) d y$, temos formalmente que

$$
\frac{\partial E_{10}}{\partial u}(x)=A(x) u(x)-(J * u)(x),
$$

onde $J * u$ é o operador convolução.

Por meio destes produtos internos, conseguimos relacionar $\Delta u$ e $A(x) u(x)-(J * u)(x)$, ou seja, obtivemos uma expressão não local para o Laplaciano com condições de contorno homogêneas de Neumann.

De fato, se consideramos a energia local

$$
E(u)=E_{0}(u)+\int_{\Omega} W(u) d x
$$

com $W^{\prime}(u)=f(u)$ e a energia não local

$$
E_{1}(u)=E_{10}(u)+\int_{\Omega} W(u) d x
$$


concluímos que

$$
\begin{aligned}
\frac{d}{d t} E(u) & =\frac{d}{d t}\left(E_{0}(u)+\int_{\Omega} W(u) d x\right) \\
& =-\int_{\Omega} u_{t} \Delta u d x+\int_{\Omega} W^{\prime}(u) u_{t} d x \\
& =-\int_{\Omega} u_{t} \Delta u d x+\int_{\Omega} f(u) u_{t} d x
\end{aligned}
$$

Desta forma, se assumimos (1.2) obtemos que

$$
u_{t}=-\frac{\partial E}{\partial u} .
$$

Por outro lado, segue da energia (1.4) que

$$
\begin{aligned}
\frac{d}{d t} E_{1}(u) & =\frac{d}{d t}\left(E_{10}(u)+\int_{\Omega} W(u) d x\right) \\
& =A(x) u(x)-(J * u)(x)+\int_{\Omega} W^{\prime}(u) u_{t} d x \\
& =A(x) u(x)-(J * u)(x)+\int_{\Omega} f(u) u_{t} d x .
\end{aligned}
$$

Isso nos permite escrever

$$
\frac{\partial E_{10}}{\partial u}=A(x) u(x)-(J * u)(x)+f(u) .
$$

Portanto, relacionamos assim a equação (1.2) com condições de contorno homogêneas de Neumann à expressão não local dada por

$$
u_{t}=J * u-A(x) u-f(u) .
$$

Essas relações são muito importantes e se justificam, pelo menos formalmente, mostrando evidências de que o problema local (1.2) e o não local (1.5) compartilham diversas propriedades, como veremos de maneira rigorosa no próximo capítulo. 


\section{Capítulo 2}

\section{Problema de difusão não local do tipo Neumann homogêneo}

Neste capítulo são apresentadas algumas propriedades do problema de difusão não local com condições de Neumann homogêneas. O estudo dessa equação foi realizado com base em [AVt10].

\subsection{Existência e unicidade de soluções}

Consideramos o problema

$$
\begin{cases}u_{t}(x, t)=\int_{\Omega} J(x-y)(u(y, t)-u(x, t)) d y, & x \in \Omega, t>0, \\ u(x, 0)=u_{0}(x), & x \in \Omega,\end{cases}
$$

com $J$ satisfazendo (H) e $\Omega$ domínio limitado em $\mathbb{R}^{N}$. Recordamos que a versão local desse problema é formalmente dada por

$$
\begin{cases}u_{t}(x, t)=\Delta u(x, t), & x \in \Omega, t>0, \\ \frac{\partial u}{\partial \eta}(x, t)=0, & x \in \partial \Omega, t>0, \\ u(x, 0)=u_{0}(x), & x \in \Omega .\end{cases}
$$

Como veremos a seguir, a equação local e sua versão não local compartilham algumas propriedades como existência e unicidade de soluções, preservação da massa total em $\Omega$ e princípios de comparação. Entretanto, não há efeito de regularização das condições iniciais [AVt10].

Definimos como solução do problema (2.1) uma função $u:[0, \infty) \rightarrow L^{1}(\Omega)$ tal que

$$
u(x, t)=e^{-A(x) t} u_{0}(x)+e^{-A(x) t} \int_{0}^{t} e^{A(x) s} \int_{\Omega} J(x-y) u(y, s) d y d s, \quad x \in \Omega, \quad t \geq 0,
$$

onde $A(x)=\int_{\Omega} J(x-y) d x$.

Verificamos que encontrar uma função que satisfaz (2.2) é equivalente a encontrar uma solução de (2.1). De fato, multiplicando a primeira equação em (2.1) por $e^{A(x) t}$, integrando em $(0, t)$ e utilizando a condição inicial, obtemos a condição (2.2). Por outro lado, se partimos da definição de solução temos

$$
\begin{aligned}
u(x, t) & =e^{-A(x) t} u_{0}(x)+e^{-A(x) t} \int_{0}^{t} e^{A(x) s} \int_{\Omega} J(x-y) u(y, s) d y d s \\
\Rightarrow e^{A(x) t} u(x, t) & =u_{0}(x)+\int_{0}^{t} e^{A(x) s} \int_{\Omega} J(x-y) u(y, s) d y d s .
\end{aligned}
$$


Derivando em relação a $t$, obtemos

$$
\begin{aligned}
& A(x) e^{A(x) t} u(x, t)+e^{A(x) t} u_{t}(x, t)=e^{A(x) t} \int_{\Omega} J(x-y) u(y, t) d y \\
\Rightarrow & u(x, t) A(x)+u_{t}(x, t)=\int_{\Omega} J(x-y) u(y, t) d y \\
\Rightarrow & u_{t}(x, t)=\int_{\Omega} J(x-y)(u(y, t)-u(x, t)) d y
\end{aligned}
$$

Portanto, procuramos uma função que satisfaça a definição (2.2). Para tanto, seguimos o raciocínio apresentado por Pérez-Llanos e Rossi em [PLR09]. Iniciamos definindo, para $t_{0}>0$ fixado, o seguinte espaço

$$
\begin{gathered}
Y_{t_{0}}=\left\{w \in C\left(\left[0, t_{0}\right], L^{1}(\Omega)\right)\right\} \text { com a norma } \\
\|w\|_{Y_{t_{0}}}=\max _{0 \leq t \leq t_{0}}\|w(\cdot, t)\|_{L^{1}(\Omega)}=\max _{0 \leq t \leq t_{0}}\left\{\int_{\Omega}|w(x, t)| d x\right\} .
\end{gathered}
$$

Nesse espaço, definimos o operador $T_{w_{0}}: Y_{t_{0}} \rightarrow Y_{t_{0}}$ por

$$
T_{w_{0}}(w)(x, t)=e^{-A(x) t} w_{0}(x)+e^{-A(x) t} \int_{0}^{t} e^{A(x) s} \int_{\Omega} J(x-y)(w(y, s)-w(x, s)) d y d s,
$$

para $x \in \Omega, t>0$ e $w_{0} \in L^{1}(\Omega)$.

A solução do problema (2.2) é um ponto fixo do operador $T_{u_{0}}$, cuja existência é garantida pelo Teorema do Ponto Fixo de Banach desde que este seja uma contração estrita e o espaço seja de Banach.

Primeiramente, verificamos que $\left(Y_{t_{0}},\|\cdot\|_{Y_{t_{0}}}\right)$ é um espaço de Banach. Tomando uma sequência de Cauchy $\left(x_{m}\right) \subset Y_{t_{0}}$, observamos que

$$
\begin{aligned}
& \left\|x_{m}-x_{n}\right\|_{Y_{t_{0}}} \rightarrow 0 \text { quando } m, n \rightarrow \infty . \\
\Rightarrow & \max _{0 \leq t \leq t_{0}}\left\|x_{m}(t)-x_{n}(t)\right\|_{L^{1}(\Omega)} \rightarrow 0 \text { quando } m, n \rightarrow \infty .
\end{aligned}
$$

Vemos então que, para todo $t \in\left[0, t_{0}\right],\left(x_{m}(t)\right)$ é uma sequência de Cauchy em $L^{1}(\Omega)$. Como $L^{1}(\Omega)$ é um espaço de Banach, a sequência converge para $x(t) \in L^{1}(\Omega)$. Para cada $t \in\left[0, t_{0}\right]$ tomamos $x(t)=\lim _{n \rightarrow \infty} x_{n}(t)$ em $L^{1}(\Omega)$.

Sejam $0 \leq t^{\prime} \leq t \leq t_{0}$. Como as funções $x_{n}(t)$ estão em $Y_{t_{0}}$, elas são contínuas em $t$. Logo, para todo $m$ fixado, dado $\varepsilon>0$, existe $\delta>0$ tal que

$$
\left|t-t^{\prime}\right| \leq \delta \Rightarrow\left|x_{m}(t)-x_{m}\left(t^{\prime}\right)\right|<\frac{\varepsilon}{3} .
$$

Pela convergência das sequências, dado $\varepsilon>0$, existe $N_{1}>0$ tal que $\left|x(t)-x_{m}(t)\right| \leq \frac{\varepsilon}{3}, \forall m>N_{1}$. Da mesma forma, existe $N_{2}>0$ tal que $\left|x_{m}\left(t^{\prime}\right)-x\left(t^{\prime}\right)\right| \leq \frac{\varepsilon}{3}, \forall m>N_{2}$. Tomemos então $N=$ $\max \left\{N_{1}, N_{2}\right\}$. Assim, para $m>N$,

$$
\left|x(t)-x_{m}(t)\right|<\frac{\varepsilon}{3} \text { e }\left|x_{m}\left(t^{\prime}\right)-x\left(t^{\prime}\right)\right|<\frac{\varepsilon}{3} .
$$

Temos

$$
\begin{aligned}
\left|x(t)-x\left(t^{\prime}\right)\right| & =\left|x(t)-x_{m}(t)+x_{m}(t)-x_{m}\left(t^{\prime}\right)+x_{m}\left(t^{\prime}\right)-x\left(t^{\prime}\right)\right| \\
& \leq\left|x(t)-x_{m}(t)\right|+\left|x_{m}(t)-x_{m}\left(t^{\prime}\right)\right|+\left|x_{m}\left(t^{\prime}\right)-x\left(t^{\prime}\right)\right| .
\end{aligned}
$$


Portanto, dado $\varepsilon>0$, existe $\delta>0$ tal que

$$
\left|t-t^{\prime}\right| \leq \delta \Rightarrow\left|x(t)-x\left(t^{\prime}\right)\right|<\frac{\varepsilon}{3}+\frac{\varepsilon}{3}+\frac{\varepsilon}{3}=\varepsilon .
$$

Logo $x \in Y_{t_{0}}$ de onde concluímos que $Y_{t_{0}}$ é um espaço vetorial normado e completo, i.e., um espaço de Banach. Além disso, obtemos que o operador $T_{w_{0}}$ está bem definido, isto é, que $T_{w_{0}}(w) \in C\left(\left[0, t_{0}\right], L^{1}(\Omega)\right)$. Primeiro, vemos que

$$
\begin{aligned}
\int_{\Omega}\left|T_{w_{0}} w(x, t)\right| d x & =\int_{\Omega}\left|e^{-A(x) t} w_{0}(x)+e^{-A(x) t} \int_{0}^{t} e^{A(x) s} \int_{\Omega} J(x-y) w(y, s) d y d s\right| d x \\
& \leq\left\|w_{0}\right\|_{L^{1}(\Omega)}+\|J\|_{\infty} \int_{\Omega} e^{A(x) t_{0}} \int_{0}^{t}\|w(s)\|_{L^{1}(\Omega)} d s d x \\
& \leq\left\|w_{0}\right\|_{L^{1}(\Omega)}+\|J\|_{\infty}|\Omega| e^{t_{0}} t_{0}\|w\|_{Y_{t_{0}}}
\end{aligned}
$$

pois $e^{-A(x) t} \leq 1$ e $e^{A(x) t}$ é crescente, dado que $A(x)$ é positivo pela hipótese (H). Aqui também utilizamos que $A(x) \leq 1$. Portanto, $T_{w_{0}} \in L^{1}(\Omega)$. Agora, como $w_{0}, w(x, t)$ e $e^{-A(x) t}$ são contínuas em $t$ temos que $T_{w_{0}} w(x, t)$ também é contínua em $t$, de onde concluímos que o operador $T_{w_{0}}$ está bem definido.

Para verificar a existência e a unicidade de solução do problema (2.1), utilizamos o lema a seguir.

Lema 2.1.1. Sejam $w_{0}, z_{0} \in L^{1}(\Omega)$ e $w, z \in Y_{t_{0}}$. Então existe uma constante $C$ dependendo apenas de $J$ e $\Omega$ tal que

$$
\left\|T_{w_{0}}(w)-T_{z_{0}}(z)\right\|_{Y_{t_{0}}} \leq C t_{0}\|w-z\|_{Y_{t_{0}}}+\left\|w_{0}-z_{0}\right\|_{L^{1}(\Omega)} .
$$

Demonstração: Temos que

$$
\begin{aligned}
& \left|\int_{\Omega} T_{w_{0}}(w)(x, t)-T_{z_{0}}(z)(x, t) d x\right| \\
= & \mid \int_{\Omega}\left(e^{-A(x) t} w_{0}(x)+e^{-A(x) t} \int_{0}^{t} e^{A(x) s} \int_{\Omega} J(x-y) w(x, s) d y d s\right. \\
- & \left.e^{-A(x) t} z_{0}(x)+e^{-A(x) t} \int_{0}^{t} e^{A(x) s} \int_{\Omega} J(x-y) z(x, s) d y d s\right) d x \mid \\
\leq & \int_{\Omega}\left|w_{0}-z_{0}\right|(x) d x+e^{t_{0}} \int_{\Omega}\left|\int_{0}^{t} \int_{\Omega} J(x-y)(w(y, s)-z(y, s)) d y d s\right| d x .
\end{aligned}
$$

Para analisar a segunda parcela da soma acima, utilizamos a desigualdade de Hölder e obtemos

$$
\begin{aligned}
& e^{t_{0}} \int_{\Omega}\left|\int_{0}^{t} \int_{\Omega} J(x-y)(w(y, s)-z(y, s)) d y d s\right| d x \leq e^{t_{0}} \int_{\Omega} \int_{0}^{t} \int_{\Omega}|J(x-y) \| w-z|(y, s) d y d s d x \\
\leq & e^{t_{0}} \int_{\Omega} \int_{0}^{t}\|J\|_{L^{\infty}(\Omega)}\|w(\cdot, s)-z(\cdot, s)\|_{L^{1}(\Omega)} d s d x \leq e^{t_{0}} \int_{\Omega} t K\|w-z\|_{Y_{t_{0}}} d x \\
\leq & e^{t_{0}} t K|\Omega|\|w-z\|_{Y t_{0}} .
\end{aligned}
$$

Tomando o máximo em $t$ nos dois lados, obtemos

$$
\left\|T_{w_{0}}(w)(x, t)-T_{z_{0}}(z)(x, t)\right\|_{Y_{t_{0}}} \leq\left\|w_{0}-z_{0}\right\|_{L^{1}(\Omega)}+\left.C t_{0}\|w-z\|\right|_{Y_{t_{0}}}
$$

provando assim a estimativa desejada.

O teorema a seguir estabelece a condição de existência e unicidade de soluções em $\Omega \times[0, \infty)$.

Teorema 2.1.1. Para cada $u_{0} \in L^{1}(\Omega)$, existe uma única solução do problema (2.1) em $[0, \infty)$. 
Demonstração: Seja $t_{0}$ tal que $C t_{0}<1$. Consideramos ainda, no lema anterior, $w_{0} \equiv z_{0} \equiv u_{0}$. Dessa forma, obtemos

$$
\left\|T_{u_{0}}(w)-\left.T_{u_{0}}(z)\right|_{Y_{t_{0}}}<C t_{0}\right\| w-z \|_{Y_{t_{0}}}
$$

$\operatorname{logo} T_{u_{0}}$ é uma contração de $Y_{t_{0}}$ em $Y_{t_{0}}$. Pelo teorema do ponto fixo de Banach, $T_{u_{0}}$ tem um ponto fixo $u \in Y_{t_{0}}$. Assim,

$$
u(x, t)=e^{-A(x) t} u_{0}(x)+e^{-A(x) t} \int_{0}^{t} e^{A(x) s} \int_{\Omega} J(x-y) u(y, s) d y d s, \quad x \in \Omega, \quad t \in\left[0, t_{0}\right] .
$$

Como visto, $u$ é solução em $\left[0, t_{0}\right]$. Para obter uma solução definida em $\left[0,2 t_{0}\right]$, podemos tomar como condição inicial a função $u\left(x, t_{0}\right)$ e aplicar o procedimento acima. Podemos então obter uma solução definida em $\left[0, n t_{0}\right]$, para todo $n$, tomando como condição inicial a função $u\left(x,(n-1) t_{0}\right)$. Assim, concluímos que existe uma única solução do problema em $[0, \infty)$.

\subsection{Comportamento assintótico das soluções}

Nesta seção é estudado o comportamento das soluções da equação não local linear. Para isto, iniciamos verificando que as soluções preservam a massa total em $\Omega$.

Teorema 2.2.1. Para cada $u_{0} \in L^{1}(\Omega)$, a única solução u do problema (2.1) preserva a massa total em $\Omega$, isto é,

$$
\int_{\Omega} u(x, t) d x=\int_{\Omega} u_{0}(x) d x \quad \forall t \in[0,+\infty) .
$$

Demonstração: Como $u$ é solução, podemos escrevê-la na forma

$$
u(x, t)=u_{0}(x)+\int_{0}^{t} \int_{\Omega} J(x-y)(u(y, s)-u(x, s)) d y d s .
$$

Integrando os dois lados da equação em $x$, aplicando o teorema de Fubini e observando que $J$ é radial, obtemos que

$$
\begin{aligned}
\int_{\Omega} u(x, t) d x & =\int_{\Omega} u_{0}(x) d x+\int_{\Omega} \int_{0}^{t} \int_{\Omega} J(x-y)(u(y, s)-u(x, s)) d y d s d x \\
& =\int_{\Omega} u_{0}(x) d x+\int_{0}^{t} \int_{\Omega} \int_{\Omega} J(x-y) u(y, s) d y d x d s-\int_{0}^{t} \int_{\Omega} \int_{\Omega} J(y-x) u(x, s) d x d y d s \\
& =\int_{\Omega} u_{0}(x) d x .
\end{aligned}
$$

Prosseguimos com um resultado referente às soluções estacionárias do problema linear. Procurar soluções estacionárias para o problema é equivalente a encontrar soluções para a equação

$$
0=\int_{\Omega} J(x-y)(\varphi(y)-\varphi(x)) d y,
$$

pois $u(x, t)=\varphi(x) \Longleftrightarrow u_{t}(x, t)=0$.

A proposição a seguir refere-se à forma das soluções estacionárias da equação linear.

Proposição 2.1. Toda solução estacionária do problema (2.1), isto é, toda função $\varphi \in L^{1}(\Omega)$ que satisfaz (2.3) é constante. 
Demonstração: A equação (2.3) implica que $\varphi$ é contínua em $\Omega$, e portanto pode ser estendida continuamente para o fecho $\bar{\Omega}$. De fato, temos que $J(0)>0$ e

$$
\varphi(x)=\frac{1}{A(x)} \int_{\Omega} J(x-y) \varphi(y) d y, \quad \forall x \in \Omega .
$$

A integral $\int_{\Omega} J(x-y) \varphi(y) d y$ é contínua devido à regularidade de $J$. Seja $g(x)=\frac{1}{A(x)}$. Em decorrência da hipótese (H) a função $J$ é uniformemente contínua em conjuntos compactos de $\mathbb{R}^{N}$. Logo, dado $\varepsilon>0$, existe $\delta>0$ tal que se $\left|x-x^{\prime}\right|<\delta$, então $\left|J(x-y)-J\left(x^{\prime}-y\right)\right|<\varepsilon$. Tem-se então

$$
\begin{aligned}
\left|A(x)-A\left(x^{\prime}\right)\right| & =\left|\int_{\Omega} J(x-y) d y-\int_{\Omega} J\left(x^{\prime}-y\right) d y\right| \\
& \leq \int_{\Omega}\left|J(x-y)-J\left(x^{\prime}-y\right)\right| d y<\varepsilon|\Omega|,
\end{aligned}
$$

de onde concluímos que $A(x)$ é contínua. Verificamos que $A(x) \geq C>0 \mathrm{em} \bar{\Omega}$. De fato, se isso não ocorre, então existe uma sequência $\left(x_{n}\right) \subset \Omega$ tal que $0 \leq A\left(x_{n}\right) \leq \frac{1}{n}$. Desta forma, vemos que $A\left(x_{n}\right) \rightarrow 0$ e podemos supor, tomando uma subsequência se necessário, que $x_{n} \rightarrow x \in \bar{\Omega}$, pois $\left(x_{n}\right) \subset \bar{\Omega}$, que é um conjunto limitado e fechado. Se $x \in \Omega, A\left(x_{n}\right) \rightarrow A(x)$ pela continuidade de $A$ e portanto

$$
0=A(x)=\int_{\Omega} J(x-y) d y>0,
$$

pois $J(0)>0$ e $J \geq 0$ por $(\mathrm{H})$ e chegamos assim a um absurdo. Caso $x \in \partial \Omega$, tem-se $\int_{\Omega} J(x-y) d y=$ 0 , o que também é absurdo por (H), uma vez que $J$ é não nula em uma vizinhança de 0 . Logo, $A(x)$ é estritamente positiva e a função $g(x)$ é contínua, o que implica na continuidade de $\varphi$. Assim $\varphi$ admite extensão contínua para $\bar{\Omega}$.

Sejam agora $K=\max _{x \in \bar{\Omega}} \varphi(x)$ e $Z=\{x \in \bar{\Omega} \mid \varphi(x)=K\}$. O conjunto $Z$ é não vazio e, por ser imagem inversa de um conjunto fechado por uma função contínua, também será fechado em $\bar{\Omega}$. Então, para provar que $Z=\bar{\Omega}$, basta provar que $Z$ é aberto em $\bar{\Omega}$. Tomamos $x_{0} \in Z$. Daí

$$
0=\int_{\Omega} J\left(x_{0}-y\right)\left(\varphi(y)-\varphi\left(x_{0}\right)\right) d y
$$

Como $J$ é não negativa, $J(0)>0$ e $\varphi(y) \leq \varphi\left(x_{0}\right)$, vale que $\varphi(y)=\varphi\left(x_{0}\right)$ para $y \in \bar{\Omega} \cap B_{\delta}\left(x_{0}\right)$ onde $\delta$ é tomado tal que $B_{\delta}(0) \subset \operatorname{supp}(J)$. Portanto, $Z$ é aberto e concluímos que $Z=\bar{\Omega}$ já que $\Omega$ é conexo. Logo $\varphi$ é constante, ou seja, $\varphi(x) \equiv K$.

Avaliamos então o comportamento das soluções em relação à solução estacionária

$$
\varphi=\frac{1}{|\Omega|} \int_{\Omega} u_{0}(x) d x
$$

Observe que

$$
\int_{\Omega} \varphi d y=\int_{\Omega} u_{0}(x) d x=\int_{\Omega} u(x, t) d x, \quad \forall \quad t>0 .
$$

Consideramos então a quantidade

$$
\beta_{1}=\inf _{\substack{u \in L^{2}(\Omega) \\ \int_{\Omega} u=0 \\ u \neq 0}} \frac{\frac{1}{2} \int_{\Omega} \int_{\Omega} J(x-y)(u(y)-u(x))^{2} d x}{\int_{\Omega} u^{2}(x) d x} .
$$

Conforme veremos, as soluções do problema convergem exponencialmente para $\varphi$ de acordo com o valor de $\beta_{1}$. Neste sentido, verificamos algumas estimativas para tal quantidade. 
Proposição 2.2. Dado $\beta_{1}$ conforme definido acima, $\beta_{1}>0$.

Demonstração: Primeiramente, temos que $\beta_{1} \geq 0$, pois $\frac{1}{2} \int_{\Omega} \int_{\Omega} J(x-y)(u(y)-u(x))^{2} d x$ e $\int_{\Omega} u^{2}(x) d x$ são não negativos. Para provar que $\beta_{1}$ é estritamente positivo, considere o conjunto $H=\left\{u \in L^{2}(\Omega) \mid \int_{\Omega} u(x) d x=0\right\}$ e o operador $T: H \rightarrow H$ dado por

$$
T(u)(x)=\int_{\Omega} J(x-y)(u(x)-u(y)) d y=-\int_{\Omega} J(x-y) u(y) d y+A(x) u(x),
$$

onde $A(x)=\int_{\Omega} J(x-y) d y$.

Temos que $T$ é auto-adjunto, pois

$$
\begin{aligned}
& <u, T v>-<T u, v> \\
& =\int_{\Omega}\left(u(x) \int_{\Omega} J(x-y)(v(x)-v(y)) d y\right) d x-\int_{\Omega}\left(v(x) \int_{\Omega} J(x-y)(u(x)-u(y)) d y\right) d x \\
& =\int_{\Omega} \int_{\Omega}(u(x) J(x-y)(v(x)-v(y))-v(x) J(x-y)(u(x)-u(y))) d x d y \\
& =\int_{\Omega} \int_{\Omega} J(x-y)(-u(x) v(y)+v(x) u(y)) d x d y \\
& =-\int_{\Omega} \int_{\Omega} J(x-y) u(x) v(y) d x d y+\int_{\Omega} \int_{\Omega} J(x-y) v(x) u(y) d x d y \\
& =-\int_{\Omega} v(y) \int_{\Omega} J(x-y) u(x) d x d y+\int_{\Omega} v(x) \int_{\Omega} J(y-x) u(y) d y d x \\
& =0,
\end{aligned}
$$

onde utilizamos o Teorema de Fubini e a simetria de $J$. O operador $T$ é a soma de dois operadores, sendo eles $B(u)(x)=A(x) u(x)$, que é inversível uma vez que $A(x)$ é estritamente positiva, e $D(u)(x)=-\int_{\Omega} J(x-y) u(y) d y$, que é compacto, por ser um operador de Hilbert-Schmidt (ver [Bre10]). Então, como $T$ é simétrico, seu espectro $\sigma(T)$ é tal que $\sigma(T) \subset[m, M]$, onde

$$
m=\inf _{\substack{u \in H \\\|u\|_{L^{2}(\Omega)}=1}}<T u, u>\text { e } M=\sup _{\substack{u \in H \\\|u\|_{L^{2}(\Omega)}=1}}<T u, u>.
$$

Vemos que

$$
\begin{aligned}
m & =\inf _{\substack{u \in H \\
\|u\|_{L^{2}(\Omega)}=1}}\langle T u, u\rangle \\
& =\inf _{\substack{u \in H \\
\|u\|_{L^{2}(\Omega)}=1}} \int_{\Omega} \int_{\Omega} J(x-y)(u(x)-u(y)) u(x) d y d x \\
& =\inf _{\substack{u \in H \\
\|u\|_{L^{2}(\Omega)}=1}} \frac{\int_{\Omega} \int_{\Omega} J(x-y)(u(x)-u(y)) u(x) d y d x}{\int_{\Omega} u^{2}(x) d x} .
\end{aligned}
$$

Mas

$$
\int_{\Omega} \int_{\Omega} J(x-y)(u(x)-u(y)) u(x) d y d x=\int_{\Omega} \int_{\Omega} J(x-y)(u(y)-u(x)) u(y) d x d y
$$


e ainda

$$
\begin{aligned}
& \int_{\Omega} \int_{\Omega} J(x-y)(u(x)-u(y)) u(x) d y d x+\int_{\Omega} \int_{\Omega} J(x-y)(u(y)-u(x)) u(y) d x d y \\
= & \int_{\Omega} \int_{\Omega} J(x-y)(u(y)-u(x))^{2} d y d x,
\end{aligned}
$$

$\log 0$

$$
\inf _{\substack{u \in H \\\|u\|_{L^{2}(\Omega)}=1}} \frac{\int_{\Omega} \int_{\Omega} J(x-y)(u(x)-u(y)) u(x) d y d x}{\int_{\Omega} u^{2}(x) d x}=\inf _{\substack{u \in H \\\|u\|_{L^{2}(\Omega)}=1}} \frac{\frac{1}{2} \int_{\Omega} \int_{\Omega} J(x-y)(u(y)-u(x))^{2} d y d x}{\int_{\Omega} u^{2}(x) d x}
$$

e portanto $m=\beta_{1}$. Sabemos que $m \geq 0$. Suponha então que $m=0$. Se isso ocorre, $0 \in \sigma(T)$, ou seja, $T$ não é inversível. Assim, $T(u)=0$ possui solução não trivial, isto é,

$$
T(u)(x)=\int_{\Omega} J(x-y)(u(x)-u(y)) d y=0
$$

para $u$ não trivial. Pela proposição $(2.1), u$ deve ser constante. Entretanto, $u \in H$, onde $H$ é o complemento ortogonal das constantes, o que nos leva a uma contradição. Portanto, $\beta_{1}>0$.

Estabelecemos agora um limite superior para o valor de $\beta_{1}$.

Lema 2.2.1. Seja $\beta_{1}$ conforme definido em (2.4). Então $\beta_{1} \leq \min _{x \in \bar{\Omega}} \int_{\Omega} J(x-y) d y$.

Demonstração: Seja $A(x)=\int_{\Omega} J(x-y) d y$. Como $\bar{\Omega}$ é compacto e $A$ é uma função contínua, então existe $x_{0} \in \bar{\Omega}$ tal que $A\left(x_{0}\right)=\min _{x \in \bar{\Omega}} A(x)$. Para cada $\varepsilon$ pequeno, sejam $B_{\varepsilon}\left(x_{1}(\varepsilon)\right)$ e $B_{\varepsilon}\left(x_{2}(\varepsilon)\right)$ duas bolas disjuntas contidas em $\Omega$, ambas com raio $\varepsilon$, com $x_{1}$ e $x_{2}$ de modo que $x_{i}(\varepsilon) \rightarrow x_{0}$, para $i=1,2$, quando $\varepsilon \rightarrow 0$. Consideramos então a função característica

$$
\chi_{B}(x)= \begin{cases}1, & \text { se } x \in B \\ 0, & \text { se } x \notin B\end{cases}
$$

que utilizamos como função teste em $\beta_{1}$. Usando $u_{\varepsilon}(x)=\chi_{B_{\varepsilon}\left(x_{1}(\varepsilon)\right)}(x)-\chi_{B_{\varepsilon}\left(x_{2}(\varepsilon)\right)}(x)$, temos que

$$
\int_{\Omega} u_{\varepsilon}(x) d x=1\left|B_{\varepsilon}(0)\right|-1\left|B_{\varepsilon}(0)\right|=0 .
$$

Vale que

$$
\begin{aligned}
\beta_{1} & \leq \frac{\frac{1}{2} \int_{\Omega} \int_{\Omega} J(x-y)\left(u_{\varepsilon}(y)-u_{\varepsilon}(x)\right)^{2} d y d x}{\int_{\Omega} u_{\varepsilon}^{2}(x) d x} \\
& =\frac{\int_{\Omega} \int_{\Omega} J(x-y)\left(u_{\varepsilon}(x)-u_{\varepsilon}(y)\right) u_{\varepsilon}(x) d y d x}{\int_{\Omega} u_{\varepsilon}^{2}(x) d x} .
\end{aligned}
$$

Por outro lado

$$
\begin{aligned}
\int_{\Omega} u_{\varepsilon}^{2}(x) d x & =\int_{\Omega} \chi_{B_{\varepsilon}\left(x_{1}(\varepsilon)\right)}^{2}(x) d x-\int_{\Omega} 2 \chi_{B_{\varepsilon}\left(x_{1}(\varepsilon)\right)}(x) \chi_{B_{\varepsilon}\left(x_{2}(\varepsilon)\right)}(x) d x+\int_{\Omega} \chi_{B_{\varepsilon}\left(x_{2}(\varepsilon)\right)}^{2}(x) d x \\
& =2\left|B_{\varepsilon}(0)\right|
\end{aligned}
$$


Logo

$$
\begin{aligned}
& \frac{\int_{\Omega} \int_{\Omega} J(x-y)\left(u_{\varepsilon}(x)-u_{\varepsilon}(y)\right) u_{\varepsilon}(x) d y d x}{\int_{\Omega} u_{\varepsilon}^{2}(x) d x} \\
= & \frac{\int_{\Omega} A(x) u_{\varepsilon}^{2}(x) d x-\int_{\Omega} \int_{\Omega} J(x-y) u_{\varepsilon}(y) u_{\varepsilon}(x) d y d x}{2\left|B_{\varepsilon}(0)\right|} .
\end{aligned}
$$

Temos ainda que

$$
\int_{\Omega} \int_{\Omega} J(x-y) u_{\varepsilon}(y) u_{\varepsilon}(x) d y d x=\int_{\Omega} u_{\varepsilon}(x) \int_{\Omega} J(x-y) u_{\varepsilon}(y) d y d x \leq \int_{\Omega} u_{\varepsilon}(x) \cdot C_{0} d x=0
$$

por (2.6). Neste caso, $C_{0}=|\Omega| K$, pois

$$
\int_{\Omega} J(x-y) u_{\varepsilon}(y) d y \leq K \int_{\Omega} u_{\varepsilon}(y) d y \leq K|\Omega| .
$$

Obtemos assim

$$
\begin{aligned}
& \frac{\int_{\Omega} A(x) u_{\varepsilon}^{2}(x) d x-\int_{\Omega} \int_{\Omega} J(x-y) u_{\varepsilon}(y) u_{\varepsilon}(x) d y d x}{2\left|B_{\varepsilon}(0)\right|} \\
= & \frac{\int_{\Omega} A(x) u_{\varepsilon}^{2}(x) d x}{2\left|B_{\varepsilon}(0)\right|} \\
= & \frac{\int_{\Omega} A(x)\left(\chi_{B_{\varepsilon}\left(x_{1}(\varepsilon)\right)}^{2}(x)+\chi_{B_{\varepsilon}\left(x_{2}(\varepsilon)\right)}^{2}(x)\right) d x}{2\left|B_{\varepsilon}(0)\right|}
\end{aligned}
$$

Tomando o limite da expressão acima, obtemos

$$
\lim _{\varepsilon \rightarrow 0} \frac{\int_{\Omega} A(x) u_{\varepsilon}^{2}(x) d x-\int_{\Omega} \int_{\Omega} J(x-y) u_{\varepsilon}(y) u_{\varepsilon}(x) d y d x}{2\left|B_{\varepsilon}(0)\right|}=A\left(x_{0}\right) .
$$

Assim, temos que $\beta_{1} \leq A\left(x_{0}\right)=\min _{x \in \bar{\Omega}} \int_{\Omega} J(x-y) d y$.

Por fim, apresentamos o resultado principal desta seção, que estima a convergência, em certo sentido, da solução da equação linear não local para o valor médio da condição inicial.

Teorema 2.2.2. Para todo $u_{0} \in L^{2}(\Omega)$, a solução $u(x, t)$ de (2.1) satisfaz

$$
\left\|u(\cdot, t)-\frac{1}{|\Omega|} \int_{\Omega} u_{0}\right\|_{L^{2}(\Omega)} \leq e^{-\beta_{1} t}\left\|u_{0}-\frac{1}{|\Omega|} \int_{\Omega} u_{0}\right\|_{L^{2}(\Omega)}
$$

para $\beta_{1}$ como em (2.4). Além disso, se $u_{0}$ é contínua e limitada, existe uma constante positiva $C>0$ tal que

$$
\left\|u(\cdot, t)-\frac{1}{|\Omega|} \int_{\Omega} u_{0}\right\|_{L^{\infty}(\Omega)} \leq C e^{-\beta_{1} t} .
$$

Demonstração: Definimos

$$
H(t)=\frac{1}{2} \int_{\Omega}\left(u(x, t)-\frac{1}{|\Omega|} \int_{\Omega} u_{0}\right)^{2} d x .
$$


Derivando a expressão acima, obtemos

$$
\begin{aligned}
H^{\prime}(t) & =\frac{1}{2} \int_{\Omega} 2\left(u(x, t)-\frac{1}{|\Omega|} \int_{\Omega} u_{0}\right) u_{t}(x, t) d x \\
& =\int_{\Omega} u(x, t) \int_{\Omega} J(x-y)(u(y, t)-u(x, t)) d y d x-\frac{1}{|\Omega|} \int_{\Omega}\left(\left(\int_{\Omega} u_{0}\right) \int_{\Omega} J(x-y)(u(y, t)-u(x, t)) d y\right) d x \\
& =\int_{\Omega} \int_{\Omega} J(x-y) u(x, t)(u(y, t)-u(x, t)) d y d x-\frac{1}{|\Omega|}\left(\int_{\Omega} u_{0}\right) \int_{\Omega} \int_{\Omega} J(x-y)(u(y, t)-u(x, t)) d y d x \\
& =\int_{\Omega} \int_{\Omega} J(x-y) u(x, t)(u(y, t)-u(x, t)) d y d x \\
& =-\frac{1}{2} \int_{\Omega} \int_{\Omega} J(x-y)(u(y, t)-u(x, t))^{2} d y d x
\end{aligned}
$$

Temos, pela conservação da massa,

$$
\begin{aligned}
\int_{\Omega}\left(u(x, t) d x-\frac{1}{|\Omega|} \int_{\Omega} u_{0}\right) d x & =\int_{\Omega} u(x, t) d x-\frac{1}{|\Omega|} \int_{\Omega}\left(\int_{\Omega} u_{0}(y) d y\right) d x \\
& =\int_{\Omega} u(x, t) d x-\frac{|\Omega|}{|\Omega|} \int_{\Omega} u_{0}(x) d x=0 .
\end{aligned}
$$

Logo, temos que $u(x, t)-\frac{1}{|\Omega|} \int_{\Omega} u_{0}=w(x, t) \in\left\{v \in L^{2}(\Omega), \int_{\Omega} v=0\right\}$. Portanto

$$
\begin{aligned}
& \frac{\frac{1}{2} \int_{\Omega} \int_{\Omega} J(x-y)\left(\left(u(y, t)-\frac{1}{|\Omega|} \int_{\Omega} u_{0}\right)-\left(u(x, t)-\frac{1}{|\Omega|} \int_{\Omega} u_{0}\right)\right)^{2} d y d x}{\int_{\Omega}\left(u(x, t)-\frac{1}{|\Omega|} \int_{\Omega} u_{0}\right)^{2} d x} \\
= & \frac{\frac{1}{2} \int_{\Omega} \int_{\Omega} J(x-y)(u(y, t)-u(x, t))^{2} d y d x}{\int_{\Omega}\left(u(x, t)-\frac{1}{|\Omega|} \int_{\Omega} u_{0}\right)^{2} d x} \\
\geq & \beta_{1},
\end{aligned}
$$

pela definição de $\beta_{1}$. Multiplicando os dois lados da desigualdade por $-\int_{\Omega}\left(u(x, t)-\frac{1}{|\Omega|} \int_{\Omega} u_{0}\right)^{2} d x$ obtemos

$$
-\frac{1}{2} \int_{\Omega} \int_{\Omega} J(x-y)(u(y, t)-u(x, t))^{2} d y d x \leq-\beta_{1} \int_{\Omega}\left(u(x, t)-\frac{1}{|\Omega|} \int_{\Omega} u_{0}\right)^{2} d x .
$$

Utilizando a expressão de $H(t)$, segue-se que

$$
H^{\prime}(t) \leq-2 \beta_{1} H(t)
$$

Integrando em $(0, t)$,

$$
H(t) \leq H(0) e^{-2 \beta_{1} t}
$$


Desta forma, observamos que

$$
\begin{aligned}
H(t) & =\frac{1}{2}\left\|u(\cdot, t)-\frac{1}{|\Omega|} \int_{\Omega} u_{0}\right\|_{L^{2}(\Omega)}^{2} \leq \frac{1}{2}\left\|u_{0}-\frac{1}{|\Omega|} \int_{\Omega} u_{0}\right\|_{L^{2}(\Omega)}^{2} e^{-2 \beta_{1} t} \\
& \Rightarrow\left\|u(\cdot, t)-\frac{1}{|\Omega|} \int_{\Omega} u_{0}\right\|_{L^{2}(\Omega)} \leq\left\|u_{0}-\frac{1}{|\Omega|} \int_{\Omega} u_{0}\right\|_{L^{2}(\Omega)} e^{-\beta_{1} t},
\end{aligned}
$$

que é a estimativa (2.7).

Para verificar a estimativa (2.8), definimos a função

$$
w(x, t)=u(x, t)-\frac{1}{|\Omega|} \int_{\Omega} u_{0},
$$

cuja norma em $L^{\infty}(\Omega)$ avaliamos.

Como o operador que define o problema (2.1) é linear, temos que $w$ é solução do problema em $H$. Logo satisfaz

$$
w(x, t)=e^{-A(x) t} w_{0}(x)+e^{-A(x) t} \int_{0}^{t} e^{A(x) s} \int_{\Omega} J(x-y) w(y, s) d y d s,
$$

onde $A(x)=\int_{\Omega} J(x-y) d y$ e $w_{0}(x)=u_{0}(x)-\frac{1}{|\Omega|} \int_{\Omega} u_{0}$.

Temos que

$$
|w(x, t)| \leq e^{-A(x) t}\left|w_{0}(x)\right|+e^{-A(x) t} \int_{0}^{t} e^{A(x) s} \int_{\Omega}|J(x-y)||w(y, s)| d y d s .
$$

Utilizando a estimativa (2.7), que se refere à norma de $w$ em $L^{2}(\Omega)$, e a desigualdade de Hölder, temos que

$$
\int_{\Omega} J(x-y)|w(y, s)| d y \leq M\left\|u_{0}-\frac{1}{|\Omega|} \int_{\Omega} u_{0}\right\| \|_{L^{2}(\Omega)} e^{-\beta_{1} s},
$$

onde $M=\max _{x \in \bar{\Omega}}\|J(x-\cdot)\|_{L^{2}(\Omega)}$. Assim

$$
|w(x, t)| \leq e^{-A(x) t}\left|w_{0}(x)\right|+C_{1} e^{-A(x) t} \int_{0}^{t} e^{A(x) s-\beta_{1} s} d s,
$$

o que nos aponta o decaimento exponencial de $w$. Pelo Lema 2.2.1, $A(x)-\beta_{1} \geq 0$. Na integral acima, se $A(x)=\beta_{1}$, a estimativa é verificada com $C=C_{1}$ pois a desigualdade é válida para todo t. Caso contrário,

$$
\int_{0}^{t} e^{A(x) s-\beta_{1} s} d s=\frac{1}{A(x)-\beta_{1}} e^{\left(A(x)-\beta_{1}\right) t}-\frac{1}{A(x)-\beta_{1}} \leq \frac{1}{A(x)-\beta_{1}} e^{\left(A(x)-\beta_{1}\right) t},
$$

e assim

$$
|w(x, t)| \leq e^{-A(x) t}\left|w_{0}(x)\right|+\frac{C e^{-\beta_{1} t}}{A(x)-\beta_{1}} .
$$

Portanto, considerando $t \rightarrow \infty$, temos que existe uma constante $C$ tal que

$$
\|w(\cdot, t)\|_{L^{\infty}(\Omega)} \leq C e^{-\beta_{1} t},
$$

conforme a estimativa (2.8). 


\subsection{Subsoluções e supersoluções}

Concluímos o capítulo com a definição de supersolução e resultados referentes à preservação de sinal das soluções.

Definição 2.3.1. Uma função $u \in C(\bar{\Omega} \times[0, T])$ é uma supersolução do problema (2.1) se

$$
u_{t}(x, t) \geq \int_{\Omega} J(x-y)(u(y, t)-u(x, t)) d y .
$$

Invertendo as desigualdades, definimos subsoluções.

Lema 2.3.1. Seja $u_{0} \geq 0$. Se $u \in C(\bar{\Omega} \times[0, T])$ é uma supersolução do problema (2.1), então $u \geq 0$.

Demonstração: Suponha que $u$ é negativa em algum ponto $\left(x_{1}, t_{1}\right)$. Consideramos a função $v(x, t)=u(x, t)+\varepsilon t$, com $\varepsilon$ tão pequeno que $v\left(x_{1}, t_{1}\right)$ ainda seja negativa. Seja $\left(x_{0}, t_{0}\right)$ o ponto de mínimo da função $v$. Como $u_{0} \geq 0$, temos que $t_{0}>0 \mathrm{e}$

$$
\begin{aligned}
v_{t}\left(x_{0}, t_{0}\right) & =u_{t}\left(x_{0}, t_{0}\right)+\varepsilon \\
& >\int_{\Omega} J\left(x_{0}-y\right)\left(u\left(y, t_{0}\right)-u\left(x_{0}, t_{0}\right)\right) d y \\
& =\int_{\Omega} J\left(x_{0}-y\right)\left(v\left(y, t_{0}\right)-v\left(x_{0}, t_{0}\right)\right) d y \geq 0,
\end{aligned}
$$

pois $J$ é positiva e $v\left(y, t_{0}\right) \geq v\left(x_{0}, t_{0}\right)$. Assim, $v_{t}\left(x_{0}, t_{0}\right)>0$, o que é uma contradição pois $\left(x_{0}, t_{0}\right)$ é ponto de mínimo de $v$. Portanto, $u \geq 0$.

Corolário 2.3.0.1. Seja $u \in C(\bar{\Omega} \times[0, t])$ uma solução do problema (2.1) com $u_{0} \geq 0$. Então $u \geq 0$.

Demonstração: Uma solução $u \in C(\bar{\Omega} \times[0, t])$ é uma supersolução do problema (2.1) que satisfaz as hipóteses do Lema 2.3.1. Portanto, $u \geq 0$.

Destacamos que Andreu-Vaillo et al. [AVt10] argumentam que vale um resultado mais geral que enunciaremos a seguir.

Lema 2.3.2. Sejam $u_{0}, v_{0} \in L^{1}(\Omega)$ com $u_{0} \geq v_{0}$. Sejam u e v soluções do problema (2.1) com condições iniciais $u_{0}$ e $v_{0}$, respectivamente. Então $u \geq v$.

Demonstração: Seja $w=u-v$. Esta função é solução do problema (2.1) com condição inicial $w_{0}=u_{0}-v_{0} \geq 0$. Considerando o Lema 2.1.1, obtemos a continuidade da solução em relação à condição inicial. Além disso, $J \in L^{\infty}\left(\mathbb{R}^{N}\right)$. Desta forma, podemos considerar que $u$ e $v$ são contínuas, o que implica na continuidade de $w$. Estamos então nas hipóteses de (2.3.1) e portanto $w \geq 0$. (ver [AVt10]).

Destacamos, entretanto, a necessidade de considerar que a solução é contínua. Isto é feito considerando a densidade de $C(\Omega)$ em $L^{1}(\Omega)$, o que nos permite afirmar que qualquer solução pode ser aproximada por uma função contínua.

Concluímos esta seção com um resultado de comparação entre subsoluções e supersoluções.

Corolário 2.3.0.2. Sejam $u \in C(\bar{\Omega} \times[0, t])$ e $v \in C(\bar{\Omega} \times[0, t])$ uma supersolução e uma subsolução do problema (2.1). Então, $u \geq v$.

Demonstração: Seja $w=u-v$. A função $w$ é supersolução do problema (2.1) com $w_{0} \equiv 0$. Daí, $w \geq 0$. 


\section{Capítulo 3}

\section{Problema com condições de Neumann e um termo de reação}

Consideramos então o problema de difusão não local com condições de contorno de Neumann e um termo de reação não linear, dado por

$$
\begin{cases}u_{t}(x, t)=\int_{\Omega} J(x-y)(u(y, t)-u(x, t)) d y+|u|^{p-1} u(x, t), & x \in \Omega, t>0, \\ u(x, 0)=u_{0}(x), & x \in \Omega,\end{cases}
$$

onde $J$ satisfaz $(\mathrm{H})$ e $p>0$. Este problema apresenta algumas propriedades semelhantes ao problema local correspondente dado por

$$
\begin{cases}u_{t}(x, t)=\Delta u(x, t)+|u|^{p-1} u(x, t), & x \in \Omega, t>0 \\ \frac{\partial u}{\partial \eta}(x, t)=0, & x \in \partial \Omega, t>0 \\ u(x, 0)=u_{0}(x), & x \in \Omega .\end{cases}
$$

A seguir, estudamos propriedades relativas ao problema (3.1), como existência e unicidade de soluções, condições de explosão, entre outros, com base na discussão apresentada por Pérez-Llanos e Rossi em [PLR09]. Vemos também que, se a condição inicial $u_{0}(x)$ for não trivial e não negativa, então a solução é não negativa em todos os pontos de $[0, T) \times \Omega$ fazendo com que o problema (3.1) seja equivalente a

$$
\begin{cases}u_{t}(x, t)=\int_{\Omega} J(x-y)(u(y, t)-u(x, t)) d y+u^{p}(x, t), & x \in \Omega, t>0, \\ u(x, 0)=u_{0}(x), & x \in \Omega,\end{cases}
$$

$\operatorname{com} p>0$.

\subsection{Existência e unicidade de soluções}

Compreendemos aqui como solução do problema (3.1) uma função $u \in C([0, T), C(\bar{\Omega}))$ que satisfaz (3.1).

Para verificarmos a existência e a unicidade de soluções utilizamos o Teorema do Ponto Fixo de Banach com base nas ideias apresentadas em [Góm14] e [PLR09]. Para tanto, fixamos $t_{0}>0$ e consideramos o espaço $X_{t_{0}}=C\left(\left[0, t_{0}\right], C(\bar{\Omega})\right)$ com a norma

$$
\|w\|_{X_{t_{0}}}=\max _{0 \leq t \leq t_{0}}\|w(\cdot, t)\|_{L^{\infty}(\bar{\Omega})}=\max _{0 \leq t \leq t_{0}} \max _{x \in \bar{\Omega}}|w(x, t)| .
$$

O espaço $X_{t_{0}}$ é de Banach. De fato, seja $\left(x_{n}\right)$ uma sequência de Cauchy em $X_{t_{0}}$. Então

$$
\left\|x_{m}-x_{n}\right\|_{X_{t_{0}}} \rightarrow 0 \text { quando } m, n \rightarrow \infty .
$$


Logo, fixado $t \in\left[0, t_{0}\right]$,

$$
\left\|x_{m}(t)-x_{n}(t)\right\|_{L^{\infty}(\Omega)} \rightarrow 0 \quad \text { quando } \quad m, n \rightarrow \infty,
$$

pois, se o máximo de $\left\|x_{m}(t)-x_{n}(t)\right\|_{L^{\infty}(\Omega)}$ em $\left[0, t_{0}\right]$ tende a zero, então o mesmo acontece para cada valor de $t$. Dessa forma, $\left(x_{n}(t)\right)$ é uma sequência de Cauchy em $C(\bar{\Omega})$.

O espaço $\left(C(\bar{\Omega}),\|\cdot\|_{\infty}\right)$ é de Banach e $x(t):=\lim _{n \rightarrow \infty} x_{n}(t)$ é o limite uniforme de uma sequência de funções contínuas, portanto é contínua. Do mesmo modo, podemos concluir que $x(t)$ é contínua em $t$. Portanto, o espaço $X_{t_{0}}$ é de Banach.

Consideramos então o operador $T_{w_{0}}: X_{t_{0}} \rightarrow X_{t_{0}}$ dado por

$$
T_{w_{0}}(w)(x, t)=e^{-A(x) t} w_{0}(x)+\int_{0}^{t} e^{-A(x)(t-s)}\left(\int_{\Omega} J(x-y) w(y, s) d y+|w|^{p-1} w(x, s)\right) d s,
$$

no qual $A(x)=\int_{\Omega} J(x-y) d y$ e $w_{0} \in C(\bar{\Omega})$. Utilizamos esse operador para verificar a existência e a unicidade de uma solução para o problema (3.1) com condição inicial $w_{0}$. Para isto, provamos o seguinte lema.

Lema 3.1.1. Seja $w_{0} \in C(\bar{\Omega})$ e considere $T_{w_{0}}: X_{t_{0}} \rightarrow X_{t_{0}}$ o operador definido por (3.3). Então $T_{w_{0}}$ está bem definido e satisfaz para todo aberto limitado $U \in X_{t_{0}}$ que

$$
\left\|T_{w_{0}}(w)-T_{z_{0}}(z)\right\|_{X_{t_{0}}} \leq\left\|w_{0}-z_{0}\right\|_{L^{\infty}}(\bar{\Omega})+C t_{0}\|w-z\|_{X_{t_{0}}}
$$

para todo $w_{0}, z_{0} \in C(\bar{\Omega})$ e todo $w, z \in U$. Além disso, para $t_{0}$ suficientemente pequeno, $T_{u_{0}}$ é uma contração estrita na bola $B\left(u_{0}, 2\left\|u_{0}\right\|_{L^{\infty}(\bar{\Omega})}\right)$.

Demonstração: Primeiramente, verificamos que $T_{w_{0}}$ está bem definido. Para todos $\left(x, t_{1}\right),\left(x, t_{2}\right) \in$ $\bar{\Omega} \times\left[0, t_{0}\right]$, com $t_{2} \geq t_{1}$, vale

$$
\begin{aligned}
& \left|T_{w_{0}}(w)\left(x, t_{2}\right)-T_{w_{0}}(w)\left(x, t_{1}\right)\right| \\
= & \left.\left|w_{0}(x)\left(e^{-A(x) t_{2}}-e^{A(x) t_{1}}\right)+\int_{t_{1}}^{t_{2}} e^{-A(x)(t-s)} \int_{\Omega} J(x-y) w(y, s) d y d s+\int_{t_{1}}^{t_{2}} e^{-A(x)(t-s)}\right| w\right|^{p-1} w(x, s) d s \mid \\
\leq & \left|w_{0}(x)\left(e^{-A(x) t_{2}}-e^{A(x) t_{1}}\right)\right|+\int_{t_{1}}^{t_{2}} e^{-A(x)(t-s)} \int_{\Omega}|J(x-y)||w(y, s)| d y d s+\int_{t_{1}}^{t_{2}} e^{-A(x)(t-s)}|w|^{p}(x, s) d s \\
\leq & \left\|w_{0}\right\|_{L^{\infty}(\Omega)}\left|e^{-A(x) t_{2}}-e^{A(x) t_{1}}\right|+\left(t_{2}-t_{1}\right) K|\Omega||| w||_{X_{0}}+\left(t_{2}-t_{1}\right)\|w\|_{X_{t_{0}}}^{p} \\
\leq & || w_{0} \|_{L^{\infty}(\Omega)}\left|e^{-A(x) t_{0}}-1\right|+\left(t_{2}-t_{1}\right) \max \{K|\Omega|, 1\}\left(\|w\|_{X_{t_{0}}}+\|w\|_{X_{t_{0}}}^{p}\right) .
\end{aligned}
$$

Utilizamos acima os fatos de que $A(x)>0$ em $\bar{\Omega}$ e que $e^{-t} \leq 1$ se $t \geq 0$. Assim, vemos que $T_{w_{0}}$ é contínua para todo $t \in\left[0, t_{0}\right]$.

Temos que $T_{w_{0}}(w)$ é contínua em $x$, pois $w_{0}$ é contínua e a convolução com $J$ também o é, visto que $w$ é contínua em $x$. Portanto, $T_{w_{0}}$ está bem definida.

Provamos então a estimativa. Sejam $w_{0}, z_{0} \in C(\bar{\Omega})$ e $w, z \in X_{t_{0}}$. Para todo $(x, t) \in \bar{\Omega} \times\left[0, t_{0}\right]$, 
vale

$$
\begin{aligned}
& \left|T_{w_{0}}(w)(x, t)-T_{z_{0}}(z)(x, t)\right| \\
= & \mid e^{-A(x) t}\left(w_{0}(x)-z_{0}(x)\right)+\int_{0}^{t} e^{-A(x)(t-s)} \int_{\Omega} J(x-y)(w(y, s)-z(y, s)) d y d s \\
+ & \int_{0}^{t} e^{-A(x)(t-s)}\left(|w|^{p-1} w(x, s)-|z|^{p-1} z(x, s)\right) d s \mid \\
\leq & e^{-A(x) t}|| w_{0}-z_{0}||_{L^{\infty}(\bar{\Omega})}+\left|\int_{0}^{t} e^{-A(x)(t-s)} \int_{\Omega} J(x-y)(w(y, s)-z(y, s)) d y d s\right| \\
+ & \left|\int_{0}^{t} e^{-A(x)(t-s)}\left(|w|^{p-1} w(x, s)-|z|^{p-1} z(x, s)\right) d s\right| \\
\leq & || w_{0}-z_{0}||_{L^{\infty}(\bar{\Omega})}+\int_{0}^{t} e^{-A(x)(t-s)} \int_{\Omega}|J(x-y)||w-z|(y, s) d y d s \\
+ & \left.\int_{0}^{t} e^{-A(x)(t-s)}|| w\right|^{p-1} w(x, s)-|z|^{p-1} z(x, s) \mid d s .
\end{aligned}
$$

Consideramos então a função $f: \mathbb{R} \rightarrow \mathbb{R}$ dada por $f(x)=|x|^{p-1} x$. Temos que

$$
f^{\prime}(x)= \begin{cases}p x^{p-1}, & \text { se } \quad x>0 \\ (-1)^{p-1} p x^{p-1}, & \text { se } \quad x<0\end{cases}
$$

Daí, pelo Teorema do Valor Médio, para $0<x<y$, existe $a \in(x, y)$ tal que

$$
\left|f^{\prime}(a)\right|=\frac{|f(x)-f(y)|}{|x-y|} \Rightarrow p\left|a^{p-1}\right||x-y|=\left.|x| x\right|^{p-1}-y|y|^{p-1} \mid .
$$

Logo, a partir dessa análise, verificamos que para cada $(x, s) \in \bar{\Omega} \times\left[0, t_{0}\right]$ existe $\eta \in(w(x, s), z(x, s))$ que satisfaz

$$
\left.|| w\right|^{p-1} w(x, s)-|z|^{p-1} z(x, s)\left|=p \eta^{p-1}\right| w(x, s)-z(x, s) \mid .
$$

Podemos então tomar $\eta \leq \max \left\{\|w\|_{X_{t_{0}}},\|z\|_{X_{t_{0}}}\right\}$ de modo que valha a desigualdade

$$
\left.|| w\right|^{p-1} w(x, s)-|z|^{p-1} z(x, s)\left|\leq p \eta^{p-1}\right| w(x, s)-z(x, s) \mid .
$$

Utilizamos ainda os fatos de que $A(x)>0$ para todo $x$ e $e^{-t} \leq 1$ se $t \geq 0$ para obter

$$
\begin{aligned}
\left|T_{w_{0}}(w)(x, t)-T_{z_{0}}(z)(x, t)\right| & \leq\left\|w_{0}-z_{0}\right\|_{L^{\infty}(\bar{\Omega})}+\int_{0}^{t} K|\Omega|\|w(\cdot, s)-z(\cdot, s)\|_{L^{\infty}(\bar{\Omega})} d s \\
& +p \eta^{p-1} \int_{0}^{t}\|w(\cdot, s)-z(\cdot, s)\|_{L^{\infty}(\bar{\Omega})} d s \\
& \leq\left\|w_{0}-z_{0}\right\|_{L^{\infty}(\bar{\Omega})}+K|\Omega| t|| w-z||_{X_{t_{0}}}+p \eta^{p-1} t\|w-z\|_{X_{t_{0}}} \\
& =\left(K|\Omega|+p \eta^{p-1}\right) t|| w-z \|_{X_{t_{0}}} .
\end{aligned}
$$

Logo, tomando o máximo nos dois lados da desigualdade, obtemos

$$
\begin{aligned}
\left\|T_{w_{0}}(w)-T_{z_{0}}(z)\right\|_{X_{t_{0}}} & \leq\left\|w_{0}-z_{0}\right\|_{L^{\infty}(\bar{\Omega})}+K|\Omega| t_{0}|| w-z\left\|_{X_{t_{0}}}+p \eta^{p-1}\left|t_{0}\right| \mid w-z\right\|_{X_{t_{0}}} \\
& =\left\|w_{0}-z_{0}\right\|_{L^{\infty}(\bar{\Omega})}+\left(K|\Omega|+p \eta^{p-1}\right) t_{0}|||w-z \||_{X_{t_{0}}} \\
& =\left\|w_{0}-z_{0}\right\|_{L^{\infty}(\bar{\Omega})}+C t_{0}|| \mid w-z \|_{X_{t_{0}}}
\end{aligned}
$$

e a estimativa está provada. 
Escolhendo então $t_{0}$ tal que $C t_{0}<\frac{1}{2}$ e $u_{0} \equiv w_{0} \equiv z_{0}$, temos

$$
\left\|T_{u_{0}}(w)-T_{u_{0}}(z)\right\|_{X_{t_{0}}} \leq C t_{0}\|w-z\|_{X_{t_{0}}}<\|w-z\|_{X_{t_{0}}} .
$$

Então, $T_{u_{0}}$ é uma contração estrita na bola $B\left(u_{0}, 2|| u_{0} \|_{L^{\infty}(\bar{\Omega})}\right)$.

A partir daqui, podemos provar resultados referentes à existência e à unicidade de soluções contínuas para o problema. Além disso, provamos uma identidade acerca da massa total em $\Omega$.

Teorema 3.1.1. Para toda função $u_{0} \in C(\bar{\Omega})$ existe uma solução $u \in C([0, T), C(\bar{\Omega}))$ de (3.1), onde $T$ é o tempo maximal de existência da solução. Se $T$ for finito, então a solução explode na norma $L^{\infty}(\bar{\Omega})$, isto é

$$
\lim _{t \rightarrow T^{-}} \sup \|u(\cdot, t)\|_{L^{\infty}(\bar{\Omega})}=\infty .
$$

Além disso, a massa total de u em $\Omega$ verifica a identidade

$$
\int_{\Omega} u(x, t) d x=\int_{\Omega} u_{0}(x) d x+\int_{0}^{t} \int_{\Omega}|u|^{p-1} u(x, s) d x d s .
$$

Demonstração: Sejam $X_{t_{0}}$ e $T_{u_{0}}$ como definidos no Lema 3.1.1. Aplicando o Teorema do Ponto Fixo de Banach ao operador $T_{u_{0}}$ verificamos que o mesmo possui apenas um ponto fixo, pois é uma contração (ver [Hön61]). Assim, existe uma única função $u \in X_{t_{0}}$ tal que

$$
u(x, t)=e^{-A(x) t} u_{0}(x)+\int_{0}^{t} e^{-A(x)(t-s)} \int_{\Omega} J(x-y) u(y, s) d y d s+\int_{0}^{t} e^{-A(x)(t-s)}|u|^{p-1} u(x, s) d s .
$$

Verificamos então que uma função é solução do problema (3.1) se e somente se for ponto fixo de $T_{u_{0}}$.

Se $u$ é ponto fixo do operador $T_{u_{0}}$, então

$$
\begin{aligned}
& u(x, t)=e^{-A(x) t} u_{0}(x)+\int_{0}^{t} \int_{\Omega} J(x-y) e^{-A(x)(t-s)} u(y, s) d y d s+\int_{0}^{t} e^{-A(x)(t-s)}|u|^{p-1} u(x, s) d s \\
& \Rightarrow e^{A(x) t} u(x, t)=u_{0}(x)+\int_{0}^{t} \int_{\Omega} J(x-y) e^{A(x) s} u(y, s) d y d s+\int_{0}^{t} e^{A(x) s}|u|^{p-1} u(x, s) \\
& \Rightarrow A(x) e^{A(x) t} u(x, t)+e^{A(x) t} u_{t}(x, t)=e^{A(x) t} \int_{\Omega} J(x-y) u(y, t) d y+e^{A(x) t}|u|^{p-1} u(x, t)
\end{aligned}
$$

e, como $e^{A(x) t} \neq 0$, temos

$$
\begin{aligned}
& A(x) u(x, t)+u_{t}(x, t)=\int_{\Omega} J(x-y) u(y, t) d y+|u|^{p-1} u(x, t) \\
& \Rightarrow u_{t}(x, t)=\int_{\Omega} J(x-y)(u(y, t)-u(x, t)) d y+|u|^{p-1} u(x, t) .
\end{aligned}
$$

Por outro lado, se $u_{t}(x, t)=\int_{\Omega} J(x-y) u(y, t) d y-A(x) u(x, t)+|u|^{p-1} u(x, t)$, então

$$
e^{A(x) t} u_{t}(x, t)=e^{A(x) t} \int_{\Omega} J(x-y) u(y, t) d y-e^{A(x) t} A(x) u(x, t)+e^{A(x) t}|u|^{p-1} u(x, t) .
$$

Integrando por partes o lado esquerdo da igualdade, temos

$$
\int_{0}^{t} e^{A(x) s} u_{t}(x, s) d s=u(x, t) e^{A(x) t}-\int_{0}^{t} e^{A(x) s} A(x) u(x, s) d s-u_{0}(x) .
$$


Obtemos assim

$$
\begin{aligned}
\begin{aligned}
& u(x, t) e^{A(x) t}-\int_{0}^{t} e^{A(x) s} A(x) u(x, s) d s-u_{0}(x)=\int_{0}^{t} e^{A(x) s} \int_{\Omega} J(x-y) u(y, s) d y d s \\
&-\int_{0}^{t} e^{A(x) s} A(x) u(x, s) d s+\int_{0}^{t} e^{A(x) s}|u|^{p-1} u(x, s) d s \\
& \Rightarrow u(x, t)=u_{0}(x) e^{-A(x) t}+\int_{0}^{t} e^{-A(x)(t-s)} \int_{\Omega} J(x-y) u(y, s) d y d s+\int_{0}^{t} e^{-A(x)(t-s)}|u|^{p-1} u(x, s) d s .
\end{aligned}
\end{aligned}
$$

Logo, se encontrarmos um ponto fixo para o operador $T_{u_{0}}$, então esta função é uma solução para o problema (3.1). Como vimos, o operador possui um único ponto fixo $u$ em $X_{t_{0}}$. Se $\|u\|_{X_{t_{0}}}<\infty$, então podemos considerar o problema

$$
\begin{cases}u_{t}(x, t)=\int_{\Omega} J(x-y)(u(y, t)-u(x, t)) d y+|u|^{p-1} u(x, t), & x \in \Omega, t \in\left[0, t_{0}\right] \\ u(x, 0)=u\left(x, t_{0}\right), & x \in \Omega .\end{cases}
$$

Dessa forma, podemos encontrar $t_{1}>t_{0}$ tal que a solução esteja definida em $\left[0, t_{1}\right)$. Se $\|u\|_{X_{t_{1}}}<$ $\infty$, então podemos continuar estendendo a solução. Se o tempo máximo de existência da solução $T$ for finito, isso indica que a norma $L^{\infty}(\bar{\Omega})$ explode em tempo finito. De fato, se isso não ocorresse, a solução poderia ser estendida para $t_{2}>T$. Logo,

$$
\lim _{t \rightarrow T^{-}} \sup \|u(\cdot, t)\|_{L^{\infty}(\Omega)}=\infty .
$$

Para obtermos o resultado referente à conservação da massa em $\Omega$, basta notarmos que, se $u$ é solução, então

$$
u(x, t)=u_{0}(x)+\int_{0}^{t} \int_{\Omega} J(x-y)(u(y, s)-u(x, s)) d y d s+\int_{0}^{t}|u|^{p-1} u(x, s) d s .
$$

Integrando os dois lados da equação em $x$ e aplicando o Teorema de Fubini, observando que $J(x-y)=J(y-x)$, obtemos

$$
\begin{aligned}
\int_{\Omega} u(x, t) d x & =\int_{\Omega} u_{0}(x) d x+\int_{0}^{t} \int_{\Omega} \int_{\Omega} J(x-y)(u(y, s)-u(x, s)) d y d x d s \\
& +\int_{\Omega} \int_{0}^{t}|u|^{p-1} u(x, s) d x d s \\
& =\int_{\Omega} u_{0}(x) d x+\int_{0}^{t} \int_{\Omega} \int_{\Omega} J(x-y) u(y, s) d y d x d s \\
& -\int_{0}^{t} \int_{\Omega} \int_{\Omega} J(y-x) u(x, s) d x d y d s+\int_{\Omega} \int_{0}^{t}|u|^{p-1} u(x, s) d s d x \\
& =\int_{\Omega} u_{0}(x) d x+\int_{0}^{t} \int_{\Omega}|u|^{p-1} u(x, s) d x d s
\end{aligned}
$$

Trataremos a seguir do Princípio de Comparação. Os argumentos aqui apresentados foram adaptados de [Góm14].

Lema 3.1.2. Seja u uma solução do problema (3.1) com condição inicial u nãa negativa e não trivial. Então $u(x, t) \geq 0 \quad \forall(x, t) \in \bar{\Omega} \times[0, T)$.

Demonstração: Seja $T_{u_{0}}$ definido como no Lema 3.1.1. Utilizamos aqui o método das aproximações sucessivas e o Teorema do Ponto Fixo de Banach conforme [Hön61]. Consideremos a sequência de funções $\left(v_{i}(x, t)\right)$ em $X_{t_{0}}$ onde $v_{1}(x, t)=u_{0}(x)$ e para $i \geq 1$ 


$$
v_{i+1}(x, t)=T_{u_{0}}\left(v_{i}(x, t)\right) .
$$

Para cada $t \in[0, T)$, tomamos $0 \leq t<t_{0}<T$ para então analisarmos o comportamento da sequência em $X_{t_{0}}$. Como $T_{u_{0}}$ é uma contração, a sequência $v_{i}(x, t)$ converge para a solução $u(x, t)$ uniformemente. Note que para verificar a afirmação do Lema é suficiente concluir que

$$
v_{n}(x, t) \geq 0 \quad \forall(x, t) \in \bar{\Omega} \times\left[0, t_{0}\right] \text { e } n \geq 1 .
$$

Provaremos essa desigualdade por indução. Para $i=1$, vale por hipótese, pois $v_{1}(x, t)=u_{0}(x) \geq$ 0 em $\Omega$. Supondo que valha para $i=k$, temos que

$$
\begin{aligned}
v_{k+1}(x, t) & =e^{-A(x) t} u_{0}(x)+\int_{0}^{t} e^{-A(x)(t-s)} \int_{\Omega} J(x-y) v_{k}(y, s) d y d s \\
& +\int_{0}^{t} e^{-A(x)(t-s)}\left|v_{k}\right|^{p-1} v_{k}(x, s) d s \geq 0,
\end{aligned}
$$

pois $J \geq 0$ e $v_{k}(x, t) \geq 0$ em $\bar{\Omega} \times[0, T)$. Portanto, $v_{k+1}(x, t) \geq 0$ para todo $(x, t) \in \bar{\Omega} \times\left[0, t_{0}\right]$.

Logo, o limite da sequência $u(x, t)$ é tal que

$$
u(x, t) \geq 0 \quad \forall(x, t) \in \bar{\Omega} \times\left[0, t_{0}\right] .
$$

A partir do Lema 3.1.2 concluímos que, quando a condição inicial $u_{0}$ for não trivial e não negativa, o problema (3.1) é equivalente ao problema (3.2), fato que passaremos a utilizar nos próximos teoremas. Prosseguimos então com um resultado análogo ao Princípio do Máximo Fraco apresentado em [Góm14].

Teorema 3.1.2. Sejam $u_{0}, v_{0} \in C(\bar{\Omega})$, ambas não negativas e não triviais. Se $u_{0}(x) \geq v_{0}(x)$ em $\bar{\Omega}$ e u e v são soluções de (3.2) com condições iniciais $u_{0}$ e $v_{0}$ respectivamente, então $u \geq v$ em $\bar{\Omega} \times[0, T)$, onde $T$ é o tempo maximal de existência de $u$.

Demonstração: Utilizamos novamente o operador $T_{u_{0}}$. Sejam $\left(w_{i}(x, t)\right)$ e $\left(z_{i}(x, t)\right)$ duas sequências de funções em $X_{t_{0}}$ tais que $w_{1}(x, t)=u_{0}(x), z_{1}(x, t)=v_{0}(x)$ e para $i \geq 1$,

$$
\begin{gathered}
w_{i+1}(x, t)=T_{u_{0}}\left(w_{i}(x, t)\right), \\
z_{i+1}(x, t)=T_{v_{0}}\left(z_{i}(x, t)\right) .
\end{gathered}
$$

Temos que $w_{n}(x, t) \rightarrow u(x, t)$ e $z_{n}(x, t) \rightarrow v(x, t)$ quando $n \rightarrow \infty$. Verificando que

$$
w_{n}(x, t) \geq z_{n}(x, t) \quad \forall(x, t) \in \bar{\Omega} \times\left[0, t_{0}\right]
$$

obteremos o resultado desejado. Procedendo novamente por indução, temos que, para $i=1$, a propriedade é válida pois $w_{1}(x, t)=u_{0}(x) \geq v_{0}(x)=z_{1}(x, t)$. Suponha que vale para $i=k$. Temos então

$$
\begin{aligned}
w_{k+1}(x, t) & =e^{-A(x) t} u_{0}(x)+\int_{0}^{t} e^{-A(x)(t-s)} \int_{\Omega} J(x-y) w_{k}(y, s) d y d s+\int_{0}^{t} e^{-A(x)(t-s)} w_{k}^{p}(x, s) d s \\
& \geq e^{-A(x) t} v_{0}(x)+\int_{0}^{t} e^{-A(x)(t-s)} \int_{\Omega} J(x-y) z_{k}(y, s) d y d s+\int_{0}^{t} e^{-A(x)(t-s)} z_{k}^{p}(x, s) d s \\
& =z_{k+1}(x, t) .
\end{aligned}
$$

Portanto, a propriedade é válida para todo $i$. Temos então que

$$
u(x, t) \geq v(x, t) \quad \forall(x, t) \in \bar{\Omega} \times\left[0, t_{0}\right] .
$$


Concluímos esta seção com a definição de subsoluções e supersoluções e um resultado referente a essas funções.

Definição 3.1.1. Uma função $\bar{u} \in C^{1}([0, T), C(\bar{\Omega}))$ é uma supersolução de (3.2) se satisfaz

$$
\left\{\begin{aligned}
\bar{u}(x, t) & \geq e^{-A(x) t} \bar{u}(x, 0)+\int_{0}^{t} e^{-A(x)(t-s)} \int_{\Omega} J(x-y)(\bar{u}(y, s)-\bar{u}(x, s)) d y d s \\
& +\int_{0}^{t} e^{-A(x)(t-s)} \bar{u}^{p}(x, s) d s \\
\bar{u}(x, 0) & \geq u_{0}(x) .
\end{aligned}\right.
$$

Invertendo as desigualdades, definimos subsoluções $\underline{u}$ para o problema.

Lema 3.1.3. Sejam $\bar{u}$ e $\underline{u}$ super e subsoluções de (3.2) respectivamente. Então, para todo $(x, t) \in$ $\bar{\Omega} \times[0, T), \bar{u}(x, t) \geq \underline{u}(x, t)$.

Demonstração: Seja $\left(v_{i}(x, t)\right)$ uma sequência de funções em $X_{t_{0}}$ com $v_{1}(x, t)=\bar{u}(x, t) \mathrm{e}$

$$
v_{i+1}(x, t)=T_{u_{0}}\left(v_{i}(x, t)\right)
$$

para $i \geq 1$. A sequência converge para $u(x, t)$ para todo $t \in\left[0, t_{0}\right]$. Verificamos então que $\bar{u}(x, t) \geq$ $v_{n}(x, t)$ em $\bar{\Omega} \times\left[0, t_{0}\right]$ para todo $n$.

Temos que $\bar{u} \geq v_{1}$, pois $v_{1}=\bar{u}$. Suponha então que vale para $i=k$. Temos

$$
\bar{u} \geq T_{u_{0}}(\bar{u}) \geq T_{u_{0}}\left(v_{k}\right)=v_{k+1},
$$

pois $T_{u_{0}}$ preserva as desigualdades entre funções. Assim, $\bar{u}(x, t) \geq u(x, t)$ para todo $t \in\left[0, t_{0}\right]$. Para todo $t \in[0, T)$ existe um intervalo $\left[0, t_{1}\right]$ tal que $t \in\left[0, t_{1}\right]$ e no qual a solução está definida. Portanto, o resultado vale para todo $t \in[0, T)$.

Um resultado análogo pode ser obtido para subsoluções invertendo as desigualdades. Assim

$$
\bar{u}(x, t) \geq u(x, t) \geq \underline{u}(x, t)
$$

em $\bar{\Omega} \times[0, T)$ e a prova está concluída.

\subsection{Condições e taxas de explosão de soluções}

O primeiro teorema que será estudado nesta seção trata das condições para a ocorrência de explosão em tempo finito da solução.

Teorema 3.2.1. Seja $u_{0} \in C(\bar{\Omega})$ não negativa e não trivial. Se $p>1$, a solução de (3.2) correspondente a $u_{0}$ explode. Se $p \leq 1$ toda solução do problema é global. Além disso, temos a estimativa para o tempo de explosão $T$

$$
T \leq \frac{1}{p-1}\left(\frac{|\Omega|}{\int_{\Omega} u_{0}(x) d x}\right)^{p-1} .
$$

Demonstração: Esta demonstração tem como base as ideias apresentadas em [PLR09]. Consideremos o caso $p>1$. Integrando a equação em $x \in \Omega$ e aplicando o Teorema de Fubini obtemos

$$
\begin{aligned}
\int_{\Omega} u_{t}(x, t) d x & =\int_{\Omega} \int_{\Omega} J(x-y)(u(y, t)-u(x, t)) d y d x+\int_{\Omega} u^{p}(x, t) d x \\
& =\int_{\Omega} u^{p}(x, t) d x
\end{aligned}
$$


Temos que $\int_{\Omega} u^{p}(x, t) d s=\|u(\cdot, t)\|_{L^{p}(\bar{\Omega})}^{p}$ e $f(x) \equiv 1 \in L^{p^{*}}(\bar{\Omega}), \operatorname{com} \frac{1}{p}+\frac{1}{p^{*}}=1, \log p^{*}=\frac{p}{p-1}$. Aplicando a desigualdade de Hölder, temos

$$
\begin{aligned}
& \|u(\cdot, t)\|_{L^{1}(\Omega)} \leq\|u(\cdot, t)\|_{L^{p}(\bar{\Omega})}\|1\|_{L^{p^{*}}(\Omega)} \\
\Rightarrow & \left(\int_{\Omega} u(x, t) d x\right)^{p} \leq\left(\int_{\Omega} u^{p}(x, t) d x\right)\left(\int_{\Omega} 1 d x\right)^{\frac{p \cdot(p-1)}{p}} \\
\Rightarrow & \left(\int_{\Omega} u(x, t) d x\right)^{p}|\Omega|^{1-p} \leq \int_{\Omega} u^{p}(x, t) d x .
\end{aligned}
$$

Consequentemente, obtemos de (3.7)

$$
\frac{\partial}{\partial t} \int_{\Omega} u(x, t) d x \geq\left(\int_{\Omega} u(x, t) d x\right)^{p}|\Omega|^{1-p} .
$$

Note que se $m(t)$ é tal que $m^{\prime}(t) \geq \lambda m^{p}(t)$, então $m$ não pode ser global. De fato, temos que $m$ é supersolução do problema ordinário $f^{\prime}(t)=\lambda f^{p}(t)$, com $p>1$. Integrando em $(0, t)$, obtemos

$$
\frac{f^{1-p}(t)}{1-p}=\lambda t+C
$$

onde $C=\frac{f^{1-p}(0)}{1-p}$. Daí

$$
\begin{gathered}
f^{p-1}(t)=\frac{1}{(\lambda t+C)(1-p)} \\
\Rightarrow f(t)=\left(\frac{1}{(\lambda t+C)(1-p)}\right)^{\frac{1}{p-1}} .
\end{gathered}
$$

Logo $f$ explode quando $t \rightarrow T_{+} \operatorname{com} T=-\frac{C}{\lambda}$. Como $m$ é supersolução da mesma equação, concluímos que $m$ também explode.

Utilizando este fato em (3.8), vemos que $\int_{\Omega} u(x, t) d x$ não pode ser global, logo $\|u\|_{L^{1}(\Omega)}$ explode em tempo finito $T$. Pela desigualdade de Hölder, $\|u\|_{L^{\infty}(\Omega)}$ também explode. Integrando a desigualdade $(3.8)$ em $(0, t)$, temos

$$
\frac{1}{1-p}\left(\frac{1}{\left(\int_{\Omega} u(x, t) d x\right)^{p-1}}-\frac{1}{\left(\int_{\Omega} u_{0}(x) d x\right)^{p-1}}\right) \geq|\Omega|^{1-p} t .
$$

Tomando o limite $t \rightarrow T$, obtemos

$$
\begin{aligned}
& -\frac{1}{1-p} \frac{1}{\left(\int_{\Omega} u_{0}(x) d x\right)^{p-1}} \geq|\Omega|^{1-p} T \\
& \Rightarrow \frac{1}{p-1} \frac{1}{\left(\int_{\Omega} u_{0}(x) d x\right)^{p-1}} \geq|\Omega|^{1-p} T \\
& \Rightarrow T \leq \frac{1}{p-1}\left(\frac{|\Omega|}{\int_{\Omega} u_{0}(x) d x}\right)^{p-1} .
\end{aligned}
$$

Provada a estimativa para $T$, verificamos agora o comportamento da solução se $p \leq 1$. Consideramos o problema auxiliar

$$
\left\{\begin{array}{l}
z^{\prime}(t)=z(t) \\
z(0)=\max _{x \in \bar{\Omega}}\left\{u_{0}(x), 1\right\}
\end{array}\right.
$$


A condição inicial garante que $z(t)>1$ para todo $t>0$. Como $p \leq 1, z(t) \geq z^{p}(t)$. Seja $v(x, t)=z(t)$ para todo $x \in \Omega$, isto é, $v$ é constante em $x$. Vale então

$$
v_{t}(x, t)=z^{\prime}(t)=z(t) \geq z^{p}(t)=v^{p}(x, t) .
$$

Multiplicando os dois lados da desigualdade por $e^{A(x) t}$ e integrando em $[0, t]$, obtemos

$$
\begin{aligned}
v(x, t) & \geq e^{-A(x) t} v(x, 0)+\int_{0}^{t} e^{-A(x)(t-s)} v^{p}(x, s) d s \\
& =\int_{0}^{t} e^{-A(x)(t-s)} \int_{\Omega} J(x-y)(v(y, s)-v(x, s)) d y d s+\int_{0}^{t} e^{-A(x)(t-s)} v^{p}(x, s) d s
\end{aligned}
$$

e $v(x, 0) \geq u_{0}(x)$. Vemos então que $v(x, t)$ é supersolução global do problema, o que nos dá $u(x, t)$ globalmente definida por comparação.

O teorema a seguir refere-se à taxa de explosão da solução e sua demonstração é uma versão detalhada da apresentada em [PLR09].

Teorema 3.2.2. Considere o problema (3.2) com $p>1, u_{0} \geq 0$ contínua e u uma solução explodindo no tempo T. Então

$$
\lim _{t \rightarrow T^{-}}(T-t)^{\frac{1}{p-1}} \max _{x \in \Omega} u(x, t)=\left(\frac{1}{p-1}\right)^{\frac{1}{p-1}} .
$$

Demonstração: Seja $T$ o tempo maximal de existência da solução, $T<\infty$. Para $t \in[0, T)$ fixado, seja $x_{0} \in \bar{\Omega}$ tal que $u\left(x_{0}, t\right)=\max _{x \in \bar{\Omega}} u(x, t)$. Neste caso, $x_{0}$ depende de $t$, mas para todo $t$ existe tal $x_{0}$. Como $u$ é solução, para $\left(x_{0}, t\right)$ vale

$$
u_{t}\left(x_{0}, t\right)=\int_{\Omega} J\left(x_{0}-y\right)\left(u(y, t)-u\left(x_{0}, t\right)\right) d y+u^{p}\left(x_{0}, t\right) .
$$

Como $u\left(x_{0}, t\right)=\max _{x \in \bar{\Omega}} u(x, t), u(y, t)-u\left(x_{0}, t\right) \leq 0$ para todo $y \in \Omega$. Portanto temos

$$
u_{t}\left(x_{0}, t\right) \leq u^{p}\left(x_{0}, t\right)
$$

Re-escrevendo a desigualdade e integrando-a em $\left(t, t_{1}\right)$, considerando que $p>1$, obtemos que

$$
\left(\frac{1}{1-p}\right)\left(\frac{1}{u^{p-1}\left(x_{0}, t_{1}\right)}-\frac{1}{u^{p-1}\left(x_{0}, t\right)}\right) \leq\left(t_{1}-t\right) .
$$

Tomando o limite $t_{1} \rightarrow T$, temos que $u\left(x_{0}, t_{1}\right) \rightarrow \infty$ e portanto

$$
\begin{array}{lc} 
& -\left(\frac{1}{1-p}\right)\left(\frac{1}{u^{p-1}\left(x_{0}, t\right)}\right) \leq(T-t) \\
\Rightarrow & \frac{1}{u^{p-1}\left(x_{0}, t\right)} \leq(T-t)(p-1) \\
\Rightarrow & u^{p-1}\left(x_{0}, t\right) \geq \frac{1}{(T-t)(p-1)} \\
\Rightarrow \quad & u\left(x_{0}, t\right) \geq(T-t)^{-\frac{1}{p-1}}\left(\frac{1}{p-1}\right)^{\frac{1}{p-1}} \\
\Rightarrow & \max _{x \in \bar{\Omega}} u(x, t) \geq(T-t)^{-\frac{1}{p-1}}\left(\frac{1}{p-1}\right)^{\frac{1}{p-1}} .
\end{array}
$$


Por outro lado, para todo $(x, t) \in \bar{\Omega} \times[0, T)$, vale

$$
\begin{aligned}
u_{t}(x, t) & =\int_{\Omega} J(x-y)(u(y, t)-u(x, t)) d y+u^{p}(x, t) \\
& =\int_{\Omega} J(x-y) u(y, t) d y-\left(\int_{\Omega} J(x-y) d y\right) u(x, t)+u^{p}(x, t)
\end{aligned}
$$

Temos que $\int_{\Omega} J(x-y) d y \leq 1$ e como $u(x, t) \geq 0, \int_{\Omega} J(x-y) u(y, t) d y \geq 0$. Logo

$$
u_{t}(x, t) \geq-u(x, t)+u^{p}(x, t)=u^{p}(x, t)\left(1-u^{-(p-1)}(x, t)\right) .
$$

Em particular, vale para $\max _{x \in \bar{\Omega}} u_{t}(x, t)$, isto é,

$$
\max _{x \in \bar{\Omega}} u_{t}(x, t) \geq \max _{x \in \bar{\Omega}} u^{p}(x, t)\left(1-\max _{x \in \bar{\Omega}} u^{-(p-1)}(x, t)\right) .
$$

De fato, se, para $t$ fixado, o máximo de $u_{t}(x, t)$ em $\bar{\Omega}$ ocorre em $x_{1}$ e o máximo de $u(x, t)$ ocorre em $x_{0}$, então

$$
\begin{aligned}
\max _{x \in \bar{\Omega}} u_{t}(x, t)=u_{t}\left(x_{1}, t\right) \geq u_{t}\left(x_{0}, t\right) & \geq u^{p}\left(x_{0}, t\right)\left(1-u^{-(p-1)}\left(x_{0}, t\right)\right) \\
& =\max _{x \in \bar{\Omega}} u^{p}(x, t)\left(1-\max _{x \in \bar{\Omega}} u^{-(p-1)}(x, t)\right) .
\end{aligned}
$$

Temos então que

$$
\max _{x \in \bar{\Omega}} u(x, t) \geq\left(\frac{1}{(p-1)(T-t)}\right)^{\frac{1}{p-1}} .
$$

Daí, obtemos

$$
\begin{aligned}
& \max _{x \in \bar{\Omega}} u^{p-1}(x, t) \geq\left(\frac{1}{(p-1)(T-t)}\right) \\
\Rightarrow & \max _{x \in \bar{\Omega}} u^{-(p-1)}(x, t) \leq(p-1)(T-t) \\
\Rightarrow & 1-\max _{x \in \bar{\Omega}} u^{-(p-1)}(x, t) \geq 1-(p-1)(T-t) .
\end{aligned}
$$

Portanto,

$$
\max _{x \in \bar{\Omega}} u_{t}(x, t) \geq \max _{x \in \bar{\Omega}} u^{p}(x, t)(1-(p-1)(T-t)) .
$$

Re-escrevendo a desigualdade e integrando-a em $\left(t, t_{1}\right)$, vemos que

$$
\max _{x \in \bar{\Omega}}\left(\frac{1}{1-p}\right)\left(u^{-p+1}\left(x, t_{1}\right)-u^{-p+1}(x, t)\right) \geq\left(t_{1}-t\right)-(p-1)\left(T t_{1}-\frac{t_{1}^{2}}{2}-T t+\frac{t^{2}}{2}\right) .
$$

Tomando o limite $t_{1} \rightarrow T$,

$$
\begin{aligned}
\frac{1}{1-p}\left(-\max _{x \in \bar{\Omega}} u^{-p+1}(x, t)\right) & \geq(T-t)-(p-1)\left(T^{2}-\frac{T^{2}}{2}-T t+\frac{t^{2}}{2}\right) \\
& =(T-t)-\frac{p-1}{2}(T-t)^{2},
\end{aligned}
$$

de onde encontramos

$$
\max _{x \in \bar{\Omega}} u^{-(p-1)}(x, t) \geq(p-1)(T-t)-\frac{1}{2}(p-1)^{2}(T-t)^{2} .
$$


Logo

$$
\max _{x \in \bar{\Omega}} u(x, t) \leq\left((p-1)(T-t)-\frac{1}{2}(p-1)^{2}(T-t)^{2}\right)^{-\frac{1}{p-1}} .
$$

Utilizando (3.10) e (3.11), vemos que

$$
\begin{aligned}
\left(\frac{1}{p-1}\right)^{\frac{1}{p-1}} & \leq \max _{x \in \bar{\Omega}} u(x, t)(T-t)^{\frac{1}{p-1}} \\
& \leq\left((p-1)(T-t)-\frac{1}{2}(p-1)^{2}(T-t)^{2}\right)^{-\frac{1}{p-1}}(T-t)^{\frac{1}{p-1}} \\
& =(T-t)^{-\frac{1}{p-1}}\left((p-1)-\frac{1}{2}(p-1)^{2}(T-t)\right)^{-\frac{1}{p-1}}(T-t)^{\frac{1}{p-1}}
\end{aligned}
$$

daí

$$
\left(\frac{1}{p-1}\right)^{\frac{1}{p-1}} \leq \max _{x \in \bar{\Omega}} u(x, t)(T-t)^{\frac{1}{p-1}} \leq\left((p-1)-\frac{1}{2}(p-1)^{2}(T-t)\right)^{-\frac{1}{p-1}} .
$$

Vale então

$$
\lim _{t \rightarrow T^{-}}\left(\frac{1}{p-1}\right)^{\frac{1}{p-1}}=\left(\frac{1}{p-1}\right)^{\frac{1}{p-1}}
$$

e

$$
\lim _{t \rightarrow T^{-}}\left((p-1)-\frac{1}{2}(p-1)^{2}(T-t)\right)^{-\frac{1}{p-1}}=\left(\frac{1}{p-1}\right)^{\frac{1}{p-1}}
$$

Logo

$$
\lim _{t \rightarrow T^{-}} \max _{x \in \bar{\Omega}} u(x, t)(T-t)^{\frac{1}{p-1}}=\left(\frac{1}{p-1}\right)^{\frac{1}{p-1}} .
$$

Assim como no problema local, a taxa de explosão é a mesma que vale para o problema ordinário $u^{\prime}=u^{p}$. Pérez-Llanos e Rossi [PLR09] destacam que o termo de difusão não tem papel determinante na taxa de explosão, fato que é observado também na dependência apenas de $p$ para que ocorra a explosão em tempo finito.

Definimos então o conjunto de explosão do problema como o conjunto de pontos do espaço onde a solução explode, isto é,

$$
B(u)=\left\{x \in \bar{\Omega}: \text { existe } x_{n} \rightarrow x, t_{n} \rightarrow T, \operatorname{com} u\left(x_{n}, t_{n}\right) \rightarrow \infty\right\} .
$$

Vemos, mediante determinadas condições, que o conjunto $B(u)$ constiste em apenas um ponto, como por exemplo é o caso do modelo local com $p>1$ e condição inicial radialmente simétrica com máximo único na origem. Nos teoremas a seguir, consideraremos o caso em que $\Omega$ é unidimensional para simplificar. Entretanto, o resultado é válido para $\mathbb{R}^{N}$, como obsevado em [PLR09].

Antes de apresentar os teoremas referentes ao conjunto de explosão, demonstramos um lema referente à simetria da solução $u$. A demonstração a seguir foi adaptada de [FPL12].

Lema 3.2.1. Considere (3.2) com $p>1$ em $B_{R}=\{|x|<R\}=\Omega$ e $J$ satisfazendo (H) tal que $\operatorname{supp}(J)=[-1,1], J \in C^{1}(\mathbb{R}, \mathbb{R})$ e monótona decrescente em $[0,1]$. Seja $u_{0} \in C^{1}\left(B_{R}\right)$ uma função simétrica não negativa com um único máximo na origem, isto é,

$$
u_{0}=u_{0}(r) \geq 0, \quad u_{0}^{\prime}(r)<0 \quad \text { se } \quad 0<r<R, \quad u_{0}^{\prime \prime}(0)<0 .
$$


Então a solução u é simétrica e $u_{x}<0$ em $(0, L] \times(0, T)$.

Demonstração: Seja $w(x, t)=u(-x, t)$. Então

$$
\begin{aligned}
w_{t}(x, t)=u_{t}(-x, t) & =\int_{-L}^{L} J(-x-y)(u(y, t)-u(-x, t)) d y+u^{p}(-x, t) \\
& =\int_{-L}^{L} J(x-(-y))(w(-y, t)-w(x, t)) d y+w^{p}(x, t),
\end{aligned}
$$

pois $J$ é par. Daí, fazendo a substituição $z=-y$, obtemos

$$
\begin{aligned}
w_{t}(x, t) & =-\int_{L}^{-L} J(x-z)(w(z, t)-w(x, t)) d z+w^{p}(x, t) \\
& =\int_{-L}^{L} J(x-z)(w(z, t)-w(x, t)) d y+w^{p}(x, t) .
\end{aligned}
$$

Portanto, como $w$ também é solução da equação, $u(x, t)=u(-x, t)$ por unicidade de soluções.

Seja agora $v=u_{x}$. Então $v$ satisfaz

$$
v_{t}(x, t)=\int_{-L}^{L} J^{\prime}(x-y)(u(y, t)-u(x, t)) d y-v(x, t) \int_{-L}^{L} J(x-y) d y+p u^{p-1}(x, t) v(x, t) .
$$

Suponha por contradição que existe um menor tempo $t_{0}>0$ e um menor valor $x_{0}>0$ tais que $v\left(x_{0}, t_{0}\right)=0$. Temos então que

$$
0 \leq v_{t}\left(x_{0}, t_{0}\right)=\int_{0}^{L} J^{\prime}\left(x_{0}-y\right)\left(u\left(y, t_{0}\right)-u\left(x_{0}, t_{0}\right)\right) d y,
$$

pois $v$ é crescente nesse ponto, uma vez que $v\left(x_{0}, 0\right)<0$. Como $\operatorname{supp}(J)=[-1,1]$, obtemos que

$$
v_{t}\left(x_{0}, t_{0}\right)=\int_{x_{0}-1}^{x_{0}+1} J^{\prime}\left(x_{0}-y\right)\left(u\left(y, t_{0}\right)-u\left(x_{0}, t_{0}\right)\right) d y .
$$

Observamos que $J^{\prime}(\alpha)<0$ se $\alpha>0$ e $J^{\prime}(\alpha)>0$ se $\alpha<0$ pelas condições de simetria e monotonicidade de $J$. Então, obtemos que $J^{\prime}\left(x_{0}-y\right)>0$ se $y>x_{0}$ e $J^{\prime}\left(x_{0}-y\right)<0$ se $y<x_{0}$. Temos que $J^{\prime}\left(x_{0}-y\right)\left(u\left(y, t_{0}\right)-u\left(x_{0}, t_{0}\right)\right)<0$ se $|y|<x_{0}$, pois neste caso $u\left(y, t_{0}\right)>u\left(x_{0}, t_{0}\right)$ uma vez que $x_{0}$ é o menor valor em que $v$ se anula. Logo,

$$
\int_{-x_{0}}^{x_{0}} J^{\prime}\left(x_{0}-y\right)\left(u\left(y, t_{0}\right)-u\left(x_{0}, t_{0}\right)\right) d y<0 .
$$

Vemos também que, se $y \in\left[x_{0}, x_{0}+1\right], J^{\prime}\left(x_{0}-y\right)\left(u\left(y, t_{0}\right)-u\left(x_{0}, t_{0}\right)\right)<0$, pois neste caso $J\left(x_{0}-y\right)>0$ e $u\left(y, t_{0}\right)-u\left(x_{0}, t_{0}\right) \leq 0$. A última afirmação decorre do fato de que $v\left(y, t_{0}\right) \leq 0$ se $y>x_{0}$. Caso contrário, pela continuidade de $u$ teríamos $t_{1}<t_{0}$ no qual $v$ se anularia. Portanto,

$$
\int_{x_{0}}^{x_{0}+1} J^{\prime}\left(x_{0}-y\right)\left(u\left(y, t_{0}\right)-u\left(x_{0}, t_{0}\right)\right) d y<0 .
$$

Analisamos então a integral em $I=\left[x_{0}-1,-x_{0}\right]$. Caso $I=\emptyset$, temos

$$
\begin{aligned}
0 & \leq v\left(x_{0}, t_{0}\right)=\int_{x_{0}-1}^{x_{0}+1} J^{\prime}\left(x_{0}-y\right)\left(u\left(y, t_{0}\right)-u\left(x_{0}, t_{0}\right)\right) d y \\
& =\int_{-x_{0}}^{x_{0}} J^{\prime}\left(x_{0}-y\right)\left(u\left(y, t_{0}\right)-u\left(x_{0}, t_{0}\right)\right) d y+\int_{x_{0}}^{x_{0}+1} J^{\prime}\left(x_{0}-y\right)\left(u\left(y, t_{0}\right)-u\left(x_{0}, t_{0}\right)\right) d y<0,
\end{aligned}
$$


chegando assim a uma contradição. Caso $I \neq \emptyset$, consideremos $I_{r}=\left[3 x_{0}, x_{0}+1\right]$ o intervalo refletido de $I$ em relação a $x_{0}$. Temos que, para todo $y \in I, u\left(y, t_{0}\right)-u\left(x_{0}, t_{0}\right)>0, J^{\prime}\left(x_{0}-y\right)>0 \mathrm{e}$ portanto a integral $\int_{x_{0}-1}^{-x_{0}} J^{\prime}\left(x_{0}-y\right)\left(u\left(y, t_{0}\right)-u\left(x_{0}, t_{0}\right)\right) d y$ é positiva. Porém, como $J^{\prime}$ é simétrica em relação à origem, para todo $y \in I$, existe $z \in I_{r}$ tal que $\left|J^{\prime}\left(x_{0}-y\right)\right|=\left|J^{\prime}\left(x_{0}-z\right)\right|$, com $\left|u\left(y, t_{0}\right)-u\left(x_{0}, t_{0}\right)\right|<\left|u\left(z, t_{0}\right)-u\left(x_{0}, t_{0}\right)\right|$. Daí obtemos que

$$
\int_{x_{0}-1}^{-x_{0}} J^{\prime}\left(x_{0}-y\right)\left(u\left(y, t_{0}\right)-u\left(x_{0}, t_{0}\right)\right) d y<-\int_{3 x_{0}}^{x_{0}+1} J^{\prime}\left(x_{0}-y\right)\left(u\left(y, t_{0}\right)-u\left(x_{0}, t_{0}\right)\right) d y .
$$

Concluímos então que

$$
\begin{aligned}
0 \leq & v\left(x_{0}, t_{0}\right)=\int_{x_{0}-1}^{x_{0}+1} J^{\prime}\left(x_{0}-y\right)\left(u\left(y, t_{0}\right)-u\left(x_{0}, t_{0}\right)\right) d y \\
= & \int_{x_{0}-1}^{-x_{0}} J^{\prime}\left(x_{0}-y\right)\left(u\left(y, t_{0}\right)-u\left(x_{0}, t_{0}\right)\right) d y+\int_{-x_{0}}^{x_{0}} J^{\prime}\left(x_{0}-y\right)\left(u\left(y, t_{0}\right)-u\left(x_{0}, t_{0}\right)\right) d y \\
& +\int_{x_{0}}^{x_{0}+1} J^{\prime}\left(x_{0}-y\right)\left(u\left(y, t_{0}\right)-u\left(x_{0}, t_{0}\right)\right) d y<0,
\end{aligned}
$$

chegando novamente a uma contradição. Portanto, $v=u_{x}<0$ em $(0, L] \times(0, T)$.

Teorema 3.2.3. (Caso Simétrico) Nas condições do Lema 3.2.1 e $p>2, B(u)=\{0\}$.

Demonstração: Esta demonstração é uma expansão das ideias apresentadas em [PLR09], adaptando argumentos utilizados por Groisman e Rossi em [GR01].

O primeiro passo é verificar que o único ponto que satisfaz a estimativa de explosão (3.9) é $x=0$.

Seja $x_{0}>0$ e $w(t)=u(0, t)-u\left(x_{0}, t\right)$. Vale, para esta função,

$$
\begin{aligned}
w^{\prime}(t) & =u_{t}(0, t)-u_{t}\left(x_{0}, t\right) \\
& =\int_{-L}^{L} J(-y)(u(y, t)-u(0, t)) d y+u^{p}(0, t)-\int_{-L}^{L} J\left(x_{0}-y\right)\left(u(y, t)-u\left(x_{0}, t\right)\right) d y-u^{p}\left(x_{0}, t\right) \\
& =\int_{-L}^{L} J(-y)(u(y, t)-u(0, t)) d y-\int_{-L}^{L} J\left(x_{0}-y\right)\left(u(y, t)-u\left(x_{0}, t\right)\right) d y+\xi^{p-1}(t) w(t),
\end{aligned}
$$

onde $\xi(t)$ é um ponto entre $u(0, t)$ e $u\left(x_{0}, t\right)$ (o Teorema do Valor Médio garante a existência deste valor). Observemos que, para todo $z \in[-L, L]$ existe um ponto $y \in\left[x_{0}-L, x_{0}+L\right]$ tal que $J(-z)=J\left(x_{0}-y\right)$, enquanto $u(z, t)-u(0, t) \geq u(y, t)-u(0, t)$. Logo, fazendo uma translação da primeira integral, obtemos

$$
\begin{aligned}
w^{\prime}(t) & \geq \int_{-L}^{L} J\left(x_{0}-y\right)\left((u(y, t)-u(0, t))-\left(u(y, t)-u\left(x_{0}, t\right)\right)\right) d y+p \xi^{p-1}(t) w(t) \\
& =\left(u(0, t)-u\left(x_{0}, t\right)\right)\left(-\int_{-L}^{L} J\left(x_{0}-y\right) d y\right)+p \xi^{p-1}(t) w(t) \\
& =w(t)\left(-\int_{-L}^{L} J\left(x_{0}-y\right) d y+1\right)-w(t)+p \xi^{p-1}(t) w(t) \\
& \geq-w(t)+p \xi^{p-1}(t) w(t) .
\end{aligned}
$$


Re-escrevendo e integrando em $\left(t_{0}, t\right)$, obtemos

$$
\begin{aligned}
& \frac{d w}{w}(t) \geq\left(-1+p \xi^{p-1}(t)\right) \\
\Rightarrow & \ln (w(t))-\ln \left(w\left(t_{0}\right)\right) \geq \int_{t_{0}}^{t}-1+p \xi^{p-1}(s) d s .
\end{aligned}
$$

Suponha por contradição que $\lim _{t \rightarrow T^{-}}(T-t)^{\frac{1}{p-1}} u\left(x_{0}, t\right)=C_{p}=\left(\frac{1}{p-1}\right)^{\frac{1}{p-1}}$. Como $x_{0}>0$, pelo Lema 3.2.1, temos $u\left(x_{0}, t\right) \leq \xi(t) \leq u(0, t)$. Daí,

$$
\lim _{t \rightarrow T^{-}}(T-t)^{\frac{1}{p-1}} u\left(x_{0}, t\right) \leq \lim _{t \rightarrow T^{-}}(T-t)^{\frac{1}{p-1}} \xi(t) \leq \lim _{t \rightarrow T^{-}}(T-t)^{\frac{1}{p-1}} u(0, t) .
$$

Como, pela simetria de $u, x=0$ é máximo de $u(x, t)$, então

$$
\lim _{t \rightarrow T^{-}}(T-t)^{\frac{1}{p-1}} u(0, t)=\left(\frac{1}{p-1}\right)^{\frac{1}{p-1}} .
$$

Logo temos que

$$
\lim _{t \rightarrow T^{-}}(T-t)^{\frac{1}{p-1}} \xi(t)=C_{p}
$$

Observamos que

$$
\begin{aligned}
\int_{t_{0}}^{t}\left(-1+p \xi^{p-1}(s)\right) d s & =\left(t_{0}-t\right)+p \int_{t_{0}}^{t} \xi^{p-1}(s) d s \\
& \geq\left(t_{0}-t\right)+p \int_{t_{0}}^{t} \frac{C_{p}^{p-1}-\varepsilon}{T-s} d s, \text { para algum } \varepsilon>0 .
\end{aligned}
$$

Mas

$$
p \int_{t_{0}}^{t} \frac{C_{p}^{p-1}-\varepsilon}{T-s} d s=-p\left(C_{p}^{p-1}-\varepsilon\right) \ln (T-t)-C_{1},
$$

onde $C_{1}=-p\left(C_{p}^{p-1}-\varepsilon\right) \ln \left(T-t_{0}\right)$.

Daí

$$
\begin{aligned}
w(t) & \geq D(T-t)^{p\left(C_{p}^{p-1}-\varepsilon\right)} \\
& =D(T-t)^{-\frac{p}{p-1}+p \varepsilon}
\end{aligned}
$$

onde $D$ depende de $w\left(t_{0}\right)$ e $C_{1}$. Note ainda que $C_{p}^{p-1}=\frac{1}{p-1}$.

Além disso,

$$
\lim _{t \rightarrow T^{-}}(T-t)^{\frac{1}{p-1}} w(t)=\lim _{t \rightarrow T^{-}}(T-t)^{\frac{1}{p-1}} u(0, t)-\lim _{t \rightarrow T^{-}}(T-t)^{\frac{1}{p-1}} u\left(x_{0}, t\right)=0 .
$$

Logo

$$
0=\lim _{t \rightarrow T^{-}}(T-t)^{\frac{1}{p-1}} w(t) \geq D \lim _{t \rightarrow T^{-}}(T-t)^{\frac{1}{p-1}-\frac{p}{p-1}+p \varepsilon}=D \lim _{t \rightarrow T^{-}}(T-t)^{-1+p \varepsilon}=\infty,
$$

o que implica que $x_{0}$ não satisfaz a taxa de explosão. Provando que o único ponto de explosão possível é a origem chegaremos ao resultado esperado. Sejam então

$$
z(x, s)=(T-t)^{\frac{1}{p-1}} u(x, t), \quad(T-t)=e^{-s}, \quad t<T .
$$


Logo, $z(x, s)=e^{\frac{s}{p-1}} u(x, t(s))$. Assim temos

$$
\begin{aligned}
z_{s}(x, s) & =\frac{\partial}{\partial s}\left(e^{-\frac{s}{p-1}} u(x, t(s))\right) \\
& =u(x, t(s)) \frac{\partial}{\partial s}\left[(T-t)^{\frac{1}{p-1}}\right]+(T-t)^{\frac{1}{p-1}} \frac{\partial}{\partial s} u(x, t(s)) \\
& =u(x, t(s)) \frac{(T-t)^{\frac{1}{p-1}-1}(-1)}{p-1}(T-t)+(T-t)^{\frac{1}{p-1}} u_{t}(x, t(s))(T-t) \\
& =-u(x, t(s)) \frac{1}{p-1}(T-t)^{\frac{1}{p-1}}+(T-t)^{\frac{1}{p-1}}(T-t) u_{t}(x, t(s))
\end{aligned}
$$

Sabemos que

$$
\begin{aligned}
u_{t}(x, t) & =\int_{-L}^{L} J(x-y)(u(y, t)-u(x, t)) d y+u^{p}(x, t) \\
& =\int_{-L}^{L} J(x-y)(z(y, s)-z(x, s))(T-t)^{-\frac{1}{p-1}} d y+z^{p}(x, s)(T-t)^{-\frac{p}{p-1}},
\end{aligned}
$$

$\log 0$

$$
\begin{aligned}
z_{s}(x, s)= & -\frac{z(x, s)}{p-1}+(T-t) \int_{-L}^{L} J(x-y)(z(y, s)-z(x, s))(T-t)^{\frac{1}{p-1}}(T-t)^{-\frac{1}{p-1}} d y \\
& +(T-t)^{\frac{1}{p-1}}(T-t) z^{p}(x, s)(T-t)^{-\frac{p}{p-1}} \\
= & -\frac{z(x, s)}{p-1}+e^{-s} \int_{-L}^{L} J(x-y)(z(y, s)-z(x, s)) d y+z^{p}(x, s) .
\end{aligned}
$$

Temos que $s>-\ln (T)$. De fato, se $s \leq-\ln (T), \ln (t) \leq 0$, absurdo. Além disso, a taxa de explosão indica que $z(x, s) \leq C_{p}<M$ para todo $s \in(-\ln (T), \infty)$, pois se $T-t>0, s>-\ln (T)$. Desta forma vemos que

$$
z(x, s)=(T-t)^{\frac{1}{p-1}} u(x, t) \leq C_{p}<M,
$$

pois

$$
\lim _{t \rightarrow T^{-}}(T-t)^{\frac{1}{p-1}} \max _{x \in \bar{\Omega}} u(x, t)=C_{p} .
$$

Verificamos que, se existe $s_{0}$ tal que $z^{p}\left(x, s_{0}\right)-\frac{1}{p-1} z\left(x, s_{0}\right)<-M e^{-s_{0}}$, então $z(x, s) \rightarrow 0$ quando $s \rightarrow \infty$.

De fato, $z$ é limitada. Além disso,

$$
\begin{aligned}
z_{s}(x, s) & =e^{-s} \int_{-L}^{L} J(x-y)(z(y, s)-z(x, s)) d y-\frac{1}{p-1} z(x, s)+z^{p}(x, s) \\
& \leq M e^{-s}-\frac{1}{p-1} z(x, s)+z^{p}(x, s),
\end{aligned}
$$

pois $M>z(y, s)$ e $z(x, s)>0, \operatorname{logo} z(y, s)-z(x, s)<M$ e $\int_{-L}^{L} J(x-y) d y \leq 1$.

Seja agora $w$ uma solução de

$$
\left\{\begin{array}{l}
w_{s}(x, s)=M e^{-s}-\frac{1}{p-1} w(x, s)+w^{p}(x, s), \\
w\left(x, s_{0}\right)=z\left(x, s_{0}\right)<M
\end{array}\right.
$$


Se existe $s_{0}$ tal que $z^{p}\left(x, s_{0}\right)-\frac{1}{p-1} z\left(x, s_{0}\right)<-M e^{-s_{0}}$, então $w_{s}\left(x, s_{0}\right)<0$, pois

$$
w_{s}\left(x, s_{0}\right)=M e^{-s_{0}}-\frac{1}{p-1} w\left(x, s_{0}\right)+w^{p}\left(x, s_{0}\right)=M e^{-s_{0}}-\frac{1}{p-1} z\left(x, s_{0}\right)+z^{p}\left(x, s_{0}\right)<0 .
$$

Suponha então que existe $s_{1}>s_{0}$ mínimo tal que $w_{s}\left(x, s_{1}\right)=0$. Vale

$$
\begin{aligned}
w_{s s}\left(x, s_{1}\right) & =-M e^{-s_{1}}-\frac{1}{p-1} w_{s}\left(x, s_{1}\right)+p w^{p-1}\left(x, s_{1}\right) w_{s}\left(x, s_{1}\right) \\
& =-M e^{-s_{1}}<0, \text { pois } M>0 .
\end{aligned}
$$

Logo $w_{s}$ é decrescente em $s_{1}$, absurdo, pois $w_{s}\left(x, s_{0}\right)<0$. Portanto, $w_{s}(x, s)<0$ para todo $s>s_{0}$. Concluímos então que $w$ é decrescente e $w(x, s)>0$ em $s>s_{0}$ pelo sinal de $z$, pois a condição inicial é positiva e caso houvesse um primeiro tempo no qual $w$ se anulasse, $w_{s}$ seria positivo neste ponto. Pelo mesmo raciocínio, $w$ não se anula.

Portanto, existe $\lim _{s \rightarrow \infty} w(x, s)=l$.

Temos que $w(x, s) \geq 0$ e $w_{s}(x, s)<0, \log w$ é limitada e obtemos assim $\lim _{s \rightarrow \infty} w_{s}(x, s)=0$.

Como

$$
\lim _{s \rightarrow \infty}-\frac{1}{p-1} w(x, s)+w^{p}(x, s)=\lim _{s \rightarrow \infty} w_{s}(x, s)-M e^{-s},
$$

tem-se

$$
-\frac{1}{p-1} l+l^{p}=0 \Rightarrow l=0 \text { ou } l^{p-1}=\frac{1}{p-1}
$$

ou ainda

$$
l=0 \text { ou } l=\left(\frac{1}{p-1}\right)^{\frac{1}{p-1}}=C_{p} .
$$

Como $w\left(s_{0}\right) \leq C_{p}$ pela definição de $z$ e $w$ é decrescente, $l \neq C_{p}$. Logo $l=0$. Por comparação, $z(x, s) \rightarrow 0$ quando $s \rightarrow \infty$.

Verificamos então que, se existe $s_{0}$ tal que $z^{p}\left(x, s_{0}\right)-\frac{1}{p-1} z\left(x, s_{0}\right)>M e^{-s_{0}}$, então $z(x, s)$ explode em tempo finito $\tilde{s}$. Vale

$$
z_{s} \geq-M e^{-s}-\frac{1}{p-1} z(x, s)+z^{p}(x, s) .
$$

Procedendo como anteriormente, obtemos que, se $w(x, s)$ é solução de

$$
w_{s}(x, s)=-M e^{-s}-\frac{1}{p-1} w(x, s)+w^{p}(x, s),
$$

$\operatorname{com} w\left(x, s_{0}\right)=z\left(x, s_{0}\right)$, temos que $w_{s}(x, s)>0$. De fato,

$$
w_{s}\left(x, s_{0}\right)=-M e^{-s_{0}}-\frac{1}{p-1} z(x, s)+z^{p}(x, s)>0 .
$$

Se houver $s_{1}>s_{0}$ tal que $w_{s}\left(x, s_{1}\right)=0$, vale

$$
\begin{aligned}
w_{s s}\left(x, s_{1}\right) & =M e^{-s_{1}}-\frac{1}{p-1} w_{s}\left(x, s_{1}\right)+p w^{p-1}(x, s) w(x, s) \\
& =M e^{-s_{1}}>0 .
\end{aligned}
$$


Portanto, $w_{s}(x, s)>0$ para todo $s>s_{0}$ e assim, $w(x, s)$ é crescente. Temos que

$$
\begin{aligned}
& w_{s}(x, s)=-M e^{-s}-\frac{1}{p-1} w(x, s)+w^{p}(x, s)>0 \\
& \Rightarrow w_{s}(x, s)+\frac{1}{p-1} w(x, s)+M e^{-s}>w^{p}(x, s) .
\end{aligned}
$$

Como $w$ é crescente, existe $\varepsilon>0$ tal que

$$
w_{s}(x, s) \geq \varepsilon w^{p}(x, s) .
$$

Como $p>2, w$ explode em tempo finito. Por comparação, $z$ explode em tempo finito $\tilde{s}$.

Para $x \neq 0, z(x, s)$ é limitado e não converge para $C_{p}$, logo $z(x, s) \rightarrow 0$. Assim, dado $\varepsilon>0$, temos

$$
z_{s}(x, s) \leq M e^{-s}-\left(\frac{1}{p-1}-\varepsilon\right) z(x, s) .
$$

Por comparação obtemos

$$
z(x, s) \leq K_{1} e^{-s}+K_{2} e^{\left(-\frac{1}{p-1}-\varepsilon\right) s} .
$$

Temos então

$$
\begin{aligned}
\left(e^{\frac{s}{p-1}} z(x, s)\right)_{s} & =e^{\frac{s}{p-1}} \frac{1}{p-1} z(x, s) \\
& +e^{\frac{s}{p-1}}\left(e^{-s} \int_{-L}^{L} J(x-y)(z(y, s)-z(x, s)) d y-\frac{1}{p-1} z(x, s)+z^{p}(x, s)\right) \\
& =e^{\frac{s}{p-1}}\left(e^{-s} \int_{-L}^{L} J(x-s)(z(y, s)-z(x, s)) d y+z^{p}(x, s)\right) .
\end{aligned}
$$

Integramos para obter a relação

$$
z(x, s)=e^{-\frac{s}{p-1}}\left(C_{1}+\int_{s_{0}}^{s} e^{-\frac{p-2}{p-1} \sigma} \int_{-L}^{L} J(x-y)(z(y, \sigma)-z(x, \sigma)) d y+e^{\sigma} z^{p}(x, \sigma) d \sigma\right)
$$

Temos que

$$
e^{\frac{s}{p}} z(x, s) \leq K_{1} e^{-s+\frac{s}{p}}+K_{2} e^{-\left(\frac{1}{p-1}-\varepsilon\right) s+\frac{s}{p}} .
$$

Observamos que

$$
-s+\frac{s}{p}=\frac{s(-p+1)}{p}<0
$$

e

$$
-\frac{1}{p-1}+\varepsilon+\frac{1}{p}=\frac{-p+\varepsilon p(p-1)+p-1}{p(p-1)}=\varepsilon-\frac{1}{p-1}<0
$$

para $\varepsilon$ suficientemente pequeno. Daí

$$
e^{s} z^{p}(x, s) \leq\left(K_{1} e^{-s+\frac{s}{p}}+K_{2} e^{-\left(\frac{1}{p-1}-\varepsilon\right) s+\frac{s}{p}}\right)^{p} \rightarrow 0 \text { quando } s \rightarrow \infty .
$$

Logo

$$
z(x, s) \leq e^{-\frac{s}{p-1}}\left(C_{1}+C_{2} \int_{s_{0}}^{s} e^{-\frac{p-2}{p-1} \sigma} d \sigma\right)
$$


Utilizando que $p>2$, temos

$$
\begin{aligned}
\int_{s_{0}}^{s} e^{-\frac{p-2}{p-1} \sigma} d \sigma & =-\frac{p-1}{p-2}\left(\left.e^{-\frac{p-2}{p-1} \sigma}\right|_{s_{0}} ^{s}\right) \\
& =-\frac{p-1}{p-2}\left(e^{-\frac{p-2}{p-1} s}-e^{-\frac{p-2}{p-1} s_{0}}\right)<\frac{p-1}{p-2} e^{-\frac{p-2}{p-1} s_{0}}=C_{3} .
\end{aligned}
$$

Portanto,

$$
z(x, s) \leq\left(C_{1}+C_{2} C_{3}\right) e^{-\frac{1}{p-1} s} .
$$

Sendo assim, para $u(x, t)$ vale

$$
u(x, t)=e^{\frac{1}{p-1} s} z(x, s) \leq\left(C_{1}+C_{2} C_{3}\right) \Rightarrow u(x, t) \text { é limitada se } x \neq 0 .
$$

O teorema a seguir apresenta um resultado mais geral acerca do comportamento da solução em relação à condição inicial.

Teorema 3.2.4. (Caso geral) Considere o problema (3.2) em um dominio geral com $p>2$. Dados $x_{0} \in \Omega$ e $\varepsilon>0$, existe uma condição inicial $u_{0}$ tal que $B(u) \subseteq B_{\varepsilon}\left(x_{0}\right)=\left\{x \in \bar{\Omega}:\left\|x-x_{0}\right\|<\varepsilon\right\}$.

Demonstração: Assim como no Teorema 3.2.3, a demonstração têm como base os argumentos apresentados em [PLR09] e [GR01]. Dado $x_{0} \in \Omega$ e $\varepsilon>0$, procuramos uma condição inicial tal que $B(u) \subseteq B_{\varepsilon}\left(x_{0}\right)$. Consideremos $u_{0}$ concentrada em uma vizinhança de $x_{0}$ e pequena longe de $x_{0}$.

Seja $\varphi$ não negativa e suave tal que

$$
\operatorname{supp}(\varphi) \subset B_{\frac{\varepsilon}{2}}\left(x_{0}\right) \text { e } \varphi(x)>0 \text { em } B_{\frac{\varepsilon}{2}}\left(x_{0}\right) .
$$

Seja então $u_{0}(x)=N \varphi(x)+\delta$. Procuramos definir $N$ e $\delta$ de modo que $u_{0}$ seja a função que procuramos.

Vale a estimativa

$$
T \leq \frac{1}{p-1}\left(\frac{|\Omega|}{\int_{\Omega} u_{0}(x) d x}\right)^{p-1} \leq \frac{C(\Omega, p, \varphi)}{N^{p-1}} .
$$

Como o tamanho de $N$ inferfere no denominador, então tomando-o suficientemente grande, obtemos $T$ tão pequeno quanto for necessário.

No Teorema 3.2.2, obtivemos a estimativa superior

$$
\begin{aligned}
\max _{x \in \bar{\Omega}} u(x, t) & \leq\left((p-1)(T-t)-\frac{1}{2}(p-1)^{2}(T-t)^{2}\right)^{-\frac{1}{p-1}} \\
& \leq C_{1}(T-t)^{-\frac{1}{p-1}}
\end{aligned}
$$

já que $(p-1)-\frac{1}{2}(p-1)^{2}(T-t)$ é limitado em intervalos limitados.

Para todo $\tilde{x} \in \Omega$,

$$
\begin{aligned}
u_{t}(\tilde{x}, t) & =\int_{\Omega} J(\tilde{x}-y)(u(y, t)-u(\tilde{x}, t)) d y+u^{p}(\tilde{x}, t) \\
& \leq \int_{\Omega} J(\tilde{x}-y) u(y, t)+u^{p}(\tilde{x}, t),
\end{aligned}
$$

pois $\int_{\Omega} J(\tilde{x}-y) u(\tilde{x}, t) d y \geq 0$. Logo,

$$
u_{t}(\tilde{x}, t) \leq C(J, \Omega, p)(T-t)^{-\frac{1}{p-1}}+u^{p}(\tilde{x}, t)
$$


De fato,

$$
\begin{aligned}
\int_{\Omega} J(\tilde{x}-y) u(y, t) d y & \leq \int_{\Omega} J(\tilde{x}-y) C_{1}(T-t)^{-\frac{1}{p-1}} d y \\
& \leq|\Omega|\|J\|_{\infty} C_{1}(T-t)^{-\frac{1}{p-1}}=C(J, \Omega, p)(T-t)^{-\frac{1}{p-1}} .
\end{aligned}
$$

Assim, $u(\tilde{x}, t)$ é subsolução de

$$
w_{t}(t)=C(J, \Omega, p)(T-t)^{-\frac{1}{p-1}}+w^{p}(t) .
$$

Seja $w(0)=\delta$ e $z(s)=e^{-\frac{s}{p-1}} w(t) \operatorname{com} s=-\ln (T-t)$. Daí, tomando $t=t(s)$ temos

$$
\begin{aligned}
z^{\prime}(s) & =-\frac{1}{p-1} e^{-\frac{s}{p-1}} w(t)+e^{-\frac{s}{p-1}} w_{t}(t) e^{-s} \\
& =-\frac{1}{p-1} e^{-\frac{s}{p-1}} w(t)+e^{-\frac{s}{p-1}}\left(C(J, \Omega, p) e^{\frac{s}{p-1}}+w^{p}(t)\right) e^{-s} \\
& =-\frac{1}{p-1} z(s)+C(J, \Omega, p) e^{-s}+e^{-\frac{s}{p-1}} w^{p}(t) e^{-s} \\
& =-\frac{1}{p-1} z(s)+C(J, \Omega, p) e^{-s}+e^{-\frac{s}{p-1} p} w^{p}(t) \\
& =-\frac{1}{p-1} z(s)+C(J, \Omega, p) e^{-s}+z^{p}(t) .
\end{aligned}
$$

Se $t=0, z(-\ln T)=T^{\frac{1}{p-1}} w(0)=T^{\frac{1}{p-1}} \delta$. Tomando $N$ grande, temos $T$ pequeno, e se $\delta$ também é pequeno, teremos $z^{\prime}(s)<0$. De fato, como

$$
z^{\prime}(s)=K e^{-s}-\frac{1}{p-1} z(s)+z^{p}(s),
$$

$\operatorname{com} K=C(J, \Omega, p)$, para $s=-\ln T$ temos

$$
z^{\prime}(s)=K T-\frac{1}{p-1} T^{\frac{1}{p-1}} \delta+T^{\frac{p}{p-1}} \delta^{p} .
$$

Como $T$ e $\delta$ são pequenos, $\delta>\delta^{p}$ e $T^{\frac{1}{p-1}}>T^{\frac{p}{p-1}}$. Daí, para $T$ suficientemente pequeno temos $z^{\prime}(-\ln T)<0$.

Suponha que exista um primeiro $s_{0}>-\ln T$ tal que $z^{\prime}\left(s_{0}\right)=0$. Então

$$
z^{\prime \prime}\left(s_{0}\right)=-K e^{-s_{0}}<0,
$$

já que $K>0$. Mas isso implica que $z^{\prime}$ é decrescente em $s_{0}$, o que é absurdo. Logo $z^{\prime}(s)<0$.

Por raciocínio análogo ao apresentado na prova do Teorema 3.2.3, em que utilizamos o problema auxiliar (3.12), concluímos que $z(s) \rightarrow 0$ quando $s \rightarrow \infty$. Ainda, $z(s) \leq K e^{-\frac{1}{p-1} s}$ pois $p>2$.

Logo, $w$ é limitada em $[0, T)$. Temos assim que $u(\tilde{x}, t)$ é limitada para todo $\tilde{x} \in \bar{\Omega} \backslash B_{\varepsilon}\left(x_{0}\right)$. Esse fato decorre da escolha $w(0)=\delta=u_{0}(x)$ se $x \in \bar{\Omega} \backslash B_{\varepsilon}\left(x_{0}\right)$. Portanto, $u$ não explode fora da vizinhança do ponto $x_{0}$ que consideramos. Logo, como sabemos que $u$ explode, $B(u) \subseteq B_{\varepsilon}\left(x_{0}\right)$.

No próximo capítulo, vemos como é possível observar os resultados aqui apresentados em simulações numéricas e conjecturar sobre outras condições mais gerais que eventualmente sejam interessantes de se considerar em trabalhos futuros. 


\section{Capítulo 4}

\section{Tratamento numérico de problemas não locais}

Neste capítulo, estudamos uma forma de analisar os problemas não locais transformando-os em problemas discretos. Apresentamos também algumas simulações numéricas, relacionando-as aos resultados apresentados nos capítulos anteriores. Na primeira seção analisamos o método de discretização e justificamos sua escolha para o tratamento dos exemplos. Já na segunda seção trazemos uma discussão sobre os problemas e condições de nosso interesse associados ao problema não local e não linear com condições de Neumann.

\subsection{Discretização do operador linear}

Esta seção é dedicada ao estudo da discretização do problema (2.1) e tem como referência os teoremas, demonstrações e definições do artigo [PLR11].

Tomando $\Omega=[0, L]$ e $0=x_{1}<\cdots<x_{N+1}=L$ uma partição, analisamos a discretização proposta por Pérez-Llanos e Rossi em [PLR11] utilizada na parte linear do problema (3.2), dada por

$$
\left\{\begin{array}{l}
u_{j}^{\prime}(t)=\sum_{i=1}^{N+1} J\left(x_{j}-x_{i}\right) h_{i}\left(u_{i}(t)-u_{j}(t)\right) \\
u_{j}(0)=u_{0}\left(x_{j}\right)
\end{array}\right.
$$

onde $h_{i}=x_{i+1}-x_{i}$ para $i=1, \cdots, N$ e $h_{N+1}=\min \left\{h_{i}, 1 \leq i \leq N\right\}$. Consideramos que $J$ satisfaz $(H), J \in C^{1}\left(\mathbb{R}^{N}, \mathbb{R}\right)$ e $\operatorname{supp}(J)=[-S, S]$. Em alguns casos a notação $A_{j}=\left\{i\right.$ tal que $\left.\left|x_{j}-x_{i}\right| \leq S\right\}$ será útil. Denotamos também $h=\max \left\{h_{i}\right\}$.

Em [PLR09], a discretização utilizada é

$$
\left\{\begin{array}{l}
u_{j}^{\prime}(t)=\sum_{i \in A_{j}} J\left(x_{j}-x_{i}\right) h_{i}\left(u_{i}(t)-u_{j}(t)\right)+u_{j}^{p}(t) \\
u_{j}(0)=u_{0}\left(x_{j}\right)
\end{array}\right.
$$

$\operatorname{com} \Omega=[-3,3], u_{0} \geq 0$ e uma partição dada por $-3=x_{-N}<\cdots<x_{N}=3$, com $N=100$. Nas simulações utilizaremos a discretização (4.1) para a parte linear e (4.2) para a equação semilinear.

Destacamos que os resultados apresentados são válidos para problemas mais gerais da forma

$$
\left\{\begin{array}{l}
u_{t}(x, t)=\int_{\Omega} J(x-y)|u(y, t)-u(x, t)|^{q-2}(u(y, t)-u(x, t)) d y, x \in \Omega, t>0 \\
u(x, 0)=u_{0}(x), x \in \Omega
\end{array}\right.
$$


utilizando a discretização

$$
\left\{\begin{aligned}
u_{j}^{\prime}(t) & =\sum_{i=1}^{N+1} J\left(x_{j}-x_{i}\right)\left|u_{i}(t)-u_{j}(t)\right|^{q-2} h_{i}\left(u_{i}(t)-u_{j}(t)\right) \\
u_{j}(0) & =u_{0}\left(x_{j}\right) .
\end{aligned}\right.
$$

A equação (4.3) é a versão não local de

$$
\begin{cases}u_{t}(x, t)=\operatorname{div}\left(|\nabla u|^{q-2} \nabla u\right), & x \in \Omega, t>0, \\ |\nabla u|^{q-2} \frac{\partial u}{\partial \eta}(x, t)=0, & x \in \partial \Omega, \\ u(x, 0)=u_{0}(x), & x \in \Omega .\end{cases}
$$

O problema (4.3) é tratado em [AVt08], onde são estabelecidas condições para existência e unicidade de soluções, convergência das soluções do problema redimensionado e comportamento assintótico das soluções.

\subsubsection{Esquema semidiscreto}

Consideremos a partição $0=x_{1}<\cdots<x_{N+1}=L$ de $\Omega \operatorname{com} h=\max \left\{h_{i}\right\}$ e o correspondente espaço de elementos finitos lineares por partes $V_{h}$ dado por

$$
V_{h}=\left\{v \in C(\Omega):\left.v\right|_{K} \in P_{1}\left(K_{j}\right) \quad \forall j=1, \cdots, N, K_{j}=\left[x_{j+1}-x_{j}\right]\right\},
$$

onde $P_{1}\left(K_{j}\right)$ denota o conjunto dos polinômios de grau menor ou igual a 1 no intervalo $K_{j}$. Consideremos também $\left\{\phi_{j}\right\}$ a base de Lagrange usual de $V_{h}$ dada por

$$
\phi_{j}(x)=\prod_{\substack{i=1 \\ i \neq j}}^{N} \frac{x-x_{i}}{x_{j}-x_{i}}
$$

(ver [KA06] para mais detalhes). Assumimos $N h=O(1)$ com $h$ tal que $x_{j+1}-x_{j} \in \operatorname{supp}(J)$ para todo $j \geq 1$, ou seja, $h_{j}<S$ e denotamos

$$
u_{h}(x, t)=\sum_{j=1}^{N+1} u_{j}(t) \phi_{j}(x)
$$

onde $u_{j}(t)$ é a solução do sistema (4.1).

Note que $u_{h}$ é a interpolação das soluções de (4.1) no intervalo $[0, L]$. A existência e unicidade locais de soluções do sistema de equações diferenciais ordinárias são garantidas pelo fato de que o lado direito da equação é Lipschitz. Observe que o sistema (4.1) é um sistema linear de equações diferenciais ordinárias que pode ser escrito como $U^{\prime}(t)=M U(t)-C U(t)$, no qual $U(t)=\left[u_{1}(t), \cdots, u_{N+1}(t)\right]^{t}, M_{j i}=J\left(x_{j}-x_{i}\right) h_{i} \mathrm{e}$

$$
C_{k j}=\left\{\begin{array}{l}
\sum_{i=1}^{N+1} J\left(x_{j}-x_{i}\right) h_{i}, \text { se } k=j \\
0, \text { se } k \neq j .
\end{array}\right.
$$

Portanto, podemos concluir que (4.1) possui soluções globais.

Este esquema é chamado de semidiscreto por utilizar uma discretização do espaço, mas preservar a continuidade do tempo. Provamos que ele converge para a solução contínua caso ela seja positiva. Iniciamos esta verificação provando que a solução do sistema satisfaz o princípio de comparação que enunciamos a seguir. 
Definição 4.1.1. Uma função contínua $\bar{U}$ será dita supersolução de (4.1) se todas as suas componentes, denotadas por $\bar{u}_{j}$, são de classe $C^{1}$ e satisfazem

$$
\left\{\begin{array}{l}
\bar{u}_{j}^{\prime}(t) \quad \geq \sum_{i \in A_{j}} J\left(x_{j}-x_{i}\right) h_{i}\left(u_{i}(t)-u_{j}(t)\right) \\
\bar{u}_{j}(0) \geq u_{0}\left(x_{j}\right) .
\end{array}\right.
$$

Definimos subsolução, denotada por $\underline{\underline{U}}$, invertendo as desigualdades.

Lema 4.1.1. Sejam $\bar{U}$ e $\underline{U}$ supersolução e subsolução de (4.1) e U a soluçãa do mesmo. Então

$$
\bar{u}_{j}(t) \geq u_{j}(t) \geq \underline{u}_{j}(t) \quad \forall t \geq 0,
$$

onde $\bar{u}_{j}, u_{j}$ e $\underline{u}_{j}$ são as componentes de $\bar{U}, U$ e $\underline{U}$, respectivamente.

Demonstração: Observe que $\bar{u}_{j}(0) \geq u_{j}(0)$ para todo $j$. Considere $\bar{v}_{j}(t)=\bar{u}_{j}(t)+\delta t+\delta$ para todo $j$ com $\delta>0$ qualquer e suponha por absurdo que existe um nó $j_{0}$ e um primeiro $t_{0}$ tal que $\bar{v}_{j_{0}}\left(t_{0}\right)=u_{j_{0}}\left(t_{0}\right)=a$. Logo em $t_{0}$ a função $\left(\bar{v}_{j_{0}}-u_{j_{0}}\right)(t)$ é não crescente. Daí obtemos

$$
\begin{aligned}
0 \geq \bar{v}_{j_{0}}^{\prime}\left(t_{0}\right)-u_{j_{0}}^{\prime}\left(t_{0}\right) & >\sum_{i \in A_{j_{0}}} J\left(x_{j_{0}}-x_{i}\right) h_{i}\left(\bar{u}_{i}\left(t_{0}\right)-\bar{u}_{j_{0}}\left(t_{0}\right)\right)-\sum_{i \in A_{j_{0}}} J\left(x_{j_{0}}-x_{i}\right) h_{i}\left(u_{i}\left(t_{0}\right)-u_{j_{0}}\left(t_{0}\right)\right) \\
& =\sum_{i \in A_{j_{0}}} J\left(x_{j_{0}}-x_{i}\right) h_{i}\left(\left(\bar{u}_{i}\left(t_{0}\right)-a\right)-\left(u_{i}\left(t_{0}\right)-a\right)\right) \\
& =\sum_{i \in A_{j_{0}}} J\left(x_{j_{0}}-x_{i}\right) h_{i}\left(\bar{u}_{i}\left(t_{0}\right)-u_{i}\left(t_{0}\right)\right) \geq 0
\end{aligned}
$$

contradição. Utilizamos aqui a suavidade da supersolução. Como $\delta$ é arbitrário, obtemos

$$
\bar{u}_{j}(t) \geq u_{j}(t) .
$$

Invertendo as desigualdades, obtemos o resultado esperado.

Note que as funções constantes $u_{i} \equiv c$ para todo $i$ são soluções do sistema. Daí, obtemos que $\max _{i} u_{i}(0) \geq u_{i}(t) \geq \min _{i} u_{i}(0)$ para todo $i$ pois a função $v$ tal que $v_{i}(t) \equiv \max _{i} u_{i}(0)$ é supersolução e a função $w$ tal que $w_{i}(t) \equiv \min _{i} u_{i}(0)$ é subsolução. Isso implica que as soluções são uniformemente limitadas.

Teorema 4.1.1. Seja u uma solução positiva de (2.1) e uh a aproximação numérica definida por (4.6), ou seja,

$$
\left\{\begin{array}{l}
u_{h}(x, t)=\sum_{j=1}^{N+1} u_{j}(t) \phi_{j}(x), \quad \text { com } \\
u_{j}^{\prime}(t)=\sum_{i \in A_{j}} J\left(x_{j}-x_{i}\right) h_{i}\left(u_{i}(t)-u_{j}(t)\right), \\
u_{j}(0)=u_{0}\left(x_{j}\right)
\end{array}\right.
$$

Então existe $K>0$ tal que, para $h>0$ suficientemente pequeno, vale

$$
\max _{0 \leq t \leq T}\left\{\max _{x_{j} \in[0, L]}\left|u\left(x_{j}, t\right)-u_{h}\left(x_{j}, t\right)\right|\right\} \leq K h .
$$


Demonstração: Sejam $v_{j}(t)=u\left(x_{j}, t\right)$ e $\xi_{j}(t)=u_{j}(t)-v_{j}(t)$ o vetor erro. Temos então

$$
\begin{aligned}
\xi_{j}^{\prime}(t) & =\sum_{i \in A_{j}} J\left(x_{j}-x_{i}\right) h_{i}\left(u_{i}(t)-u_{j}(t)\right)+\sum_{i \in A_{j}} J\left(x_{j}-x_{i}\right) h_{i}\left(v_{i}(t)-v_{j}(t)\right) \\
& -\sum_{i \in A_{j}} J\left(x_{j}-x_{i}\right) h_{i}\left(v_{i}(t)-v_{j}(t)\right)-\int_{\Omega} J\left(x_{j}-y\right)\left(u(y, t)-u\left(x_{j}, t\right)\right) d y \\
& =\sum_{i \in A_{j}} J\left(x_{j}-x_{i}\right) h_{i}\left(v_{i}(t)-v_{j}(t)\right)-\int_{\Omega} J\left(x_{j}-y\right)\left(u(y, t)-u\left(x_{j}, t\right)\right) d y \\
& +\sum_{i \in A_{j}} J\left(x_{j}-x_{i}\right) h_{i}\left(\xi_{i}(t)-\xi_{j}(t)\right) .
\end{aligned}
$$

Note que

$$
\sum_{i=1}^{N+1} J\left(x_{j}-x_{i}\right) h_{i}\left(v_{i}(t)-v_{j}(t)\right)=\sum_{i=1}^{N} J\left(x_{j}-x_{i}\right) h_{i}\left(v_{i}(t)-v_{j}(t)\right)+J\left(x_{j}-x_{N+1}\right) h_{N+1}\left(v_{N+1}(t)-v_{j}(t)\right) .
$$

Como $J\left(x_{j}-x_{N+1}\right) h_{N+1}\left(v_{N+1}(t)-v_{j}(t)\right) \leq M h$, com $M$ constante, precisamos apenas estimar a diferença

$$
\sum_{i=1}^{N} J\left(x_{j}-x_{i}\right) h_{i}\left(v_{i}(t)-v_{j}(t)\right)-\int_{\Omega} J\left(x_{j}-y\right)\left(u(y, t)-u\left(x_{j}, t\right)\right) d y .
$$

Para isto consideramos $f_{i}^{j}(x)=\int_{x_{i}}^{x} J\left(x_{j}-y\right)\left(u(y, t)-u\left(x_{j}, t\right)\right) d y$ e logo temos

$$
\int_{\Omega} J\left(x_{j}-y\right)\left(u(y, t)-u\left(x_{j}, t\right)\right) d y=\sum_{i=1}^{N} f_{i}^{j}\left(x_{i+1}\right), \quad i=1, \cdots, N
$$

Assumindo que $J$ e $u$ sejam suficientemente regulares, expandimos a função $f_{i}^{j}$ em série de Taylor em torno de $x_{i}$ e obtemos

$$
\begin{aligned}
f_{i}^{j}\left(x_{i+1}\right) & =f_{i}^{j}\left(x_{i}\right)+h_{i} J\left(x_{j}-x_{i}\right)\left(u\left(x_{i}, t\right)-u\left(x_{j}, t\right)\right) \\
& +\frac{1}{2} h_{i}^{2}\left(J^{\prime}\left(x_{j}-\alpha_{i}\right)\left(u\left(\alpha_{i}, t\right)-u\left(x_{j}, t\right)\right)+J\left(x_{j}-\alpha_{i}\right) \partial_{x}\left(u\left(\alpha_{i}, t\right)-u\left(x_{j}, t\right)\right)\right),
\end{aligned}
$$

para algum $\alpha_{i} \in\left[x_{i}, x_{j}\right]$. Daí,

$$
\begin{aligned}
\sum_{i=1}^{N} f_{i}^{j}\left(x_{i+1}\right) & =\sum_{i \in A_{j}} h_{i} J\left(x_{j}-x_{i}\right)\left(u\left(x_{i}, t\right)-u\left(x_{j}, t\right)\right) \\
& +\frac{1}{2} h_{i}^{2}\left(J^{\prime}\left(x_{j}-\alpha_{i}\right)\left(u\left(\alpha_{i}, t\right)-u\left(x_{j}, t\right)\right)+J\left(x_{j}-\alpha_{i}\right) \partial_{x}\left(u\left(\alpha_{i}, t\right)-u\left(x_{j}, t\right)\right)\right) \\
& \leq \sum_{i \in A_{j}} h_{i} J\left(x_{j}-x_{i}\right)\left(v_{i}(t)-v_{j}(t)\right)+D_{j} h_{i}^{2},
\end{aligned}
$$

onde $D_{j}=\frac{1}{2} \max _{0 \leq t \leq T} \max _{z \in \Omega}\left\{J^{\prime}\left(x_{j}-z\right)\left(u(z, t)-u\left(x_{j}, t\right)\right)+J\left(x_{j}-z\right) \partial_{x}\left(u(z, t)-u\left(x_{j}, t\right)\right)\right\}$. Como $N$ e $h$ são inversamente proporcionais, temos que

$$
\begin{gathered}
O\left(h_{i}^{2}\right)=D_{j} h_{i}^{2} \leq D_{j} h^{2} \\
\Rightarrow \sum_{i \in A_{j}} O\left(h_{i}^{2}\right) \leq N \cdot D \cdot h^{2},
\end{gathered}
$$


onde $D=\max D_{j}$. Mas $N h \leq K$ para algum $K$ fixo, logo

$$
\sum_{i \in A_{j}} O\left(h_{i}^{2}\right) \leq K \cdot D \cdot h
$$

e portanto a aproximação tem erro $O(h)$.

Logo, o vetor $\xi(t)$ satisfaz

$$
\left\{\begin{array}{l}
\xi_{j}^{\prime}(t)=\sum_{i \in A_{j}} J\left(x_{j}-x_{i}\right) h_{i}\left(\xi_{i}(t)-\xi_{j}(t)\right)+C_{j} h \\
\xi_{j}(0)=0
\end{array}\right.
$$

onde $C_{j}$ é dado pelas funções $f_{i}^{j}$.

Seja $C=\max _{j, x, t} C_{j}$ e $w_{j}(t)=w(t)=C h t$, para todo $i=1, \cdots, N+1$. Daí vale

$$
w_{j}^{\prime}(t)=C h \geq \sum_{i \in A_{j}} J\left(x_{j}-x_{i}\right) h_{i}(C-C) h t+C_{j} h=C_{j} h .
$$

Seja $E(t)$ solução de (4.7). Então $w(t) \geq E(t)$ para todo $t$. De fato, suponha por absurdo que existe um primeiro $t_{0}$ tal que $\bar{w}\left(t_{0}\right)-E\left(t_{0}\right)=0$, onde $\bar{w}(t)=w(t)+\delta t+\delta$ com $\delta>0$, ou seja, $E\left(t_{0}\right)=\bar{w}\left(t_{0}\right)$. Logo

$$
\begin{aligned}
0 & \geq \bar{w}^{\prime}\left(t_{0}\right)-E^{\prime}\left(t_{0}\right) \\
& >\sum_{i \in A_{j}} J\left(x_{j}-x_{i}\right) h_{i}\left(w_{i}-w_{j}\right)\left(t_{0}\right)-\sum_{i \in A_{j}} J\left(x_{j}-x_{i}\right) h_{i}\left(E_{i}-E_{j}\right)\left(t_{0}\right) \\
& =\sum_{i \in A_{j}} J\left(x_{j}-x_{i}\right) h_{i}\left(w_{i}-E_{i}\right)\left(t_{0}\right) \geq 0,
\end{aligned}
$$

absurdo. Logo, $\xi_{j}(t) \leq w(t) \leq K h$, com $K=C T$, para todo $t \leq T$, para todo $j=1, \cdots, N+1$. Aplicando o mesmo raciocínio para o vetor $-\xi_{j}(t)$, obtemos

$$
\left|\xi_{j}(t)\right| \leq K h \Rightarrow \max _{0 \leq t \leq T}\left\{\max _{x_{j} \in[0, L]}\left|u\left(x_{j}, t\right)-u_{h}\left(x_{j}, t\right)\right|\right\} \leq K h .
$$

Provamos então que o esquema semidiscreto converge para a solução do problema. O lema a seguir refere-se à preservação de simetria ao aproximarmos a solução por meio da discretização (4.1). Este resultado será visível nas simulações apresentadas em seções posteriores.

Lema 4.1.2. Seja $u_{0}(x)$ uma condição inicial simétrica em um intervalo $[-L, L]$ e $\left\{x_{i}\right\}$ uma partição também simétrica, $-N-1 \leq i \leq N+1$ com $x_{i}=x_{-i}$. Então

$$
u_{i}(t)=u_{-i}(t) .
$$

Demonstração: Seja $w_{j}(t)=u_{-j}(t)$. Vale

$$
\left\{\begin{array}{l}
w_{j}^{\prime}(t)=\sum_{i \in A_{-j}} J\left(x_{-j}-x_{i}\right) h_{i}\left(u_{i}(t)-w_{j}(t)\right), \\
w_{j}(0)=u_{0}\left(x_{-j}\right)=u_{0}\left(x_{j}\right) .
\end{array}\right.
$$

Mas $A_{-j}=-A_{j}$ pela simetria da partição. De fato, se $k \in A_{j},\left|x_{k}-x_{j}\right| \leq S,\left|x_{-k}-x_{-j}\right| \leq S$ e 
assim $-k \in A_{-j}$. Como $J$ é simétrica, re-escrevemos a equação como

$$
\begin{aligned}
w_{j}^{\prime}(t) & =\sum_{i \in A_{j}} J\left(x_{-j}-x_{-i}\right) h_{i}\left(u_{-i}(t)-w_{j}(t)\right) \\
& =\sum_{i \in A_{j}} J\left(x_{j}-x_{i}\right) h_{i}\left(w_{i}(t)-w_{j}(t)\right) .
\end{aligned}
$$

Pela unicidade de soluções, a equação é satisfeita se e somente se $u_{i}(t)=u_{-i}(t)$.

A seguir, verificaremos como podemos obter soluções para o sistema de EDOs discretizando a variável temporal.

\subsubsection{Esquema totalmente discreto}

Utilizamos o Método de Euler para obter uma discretização do sistema de EDOs (4.1), obtendo o que chamaremos de esquema totalmente discreto. Temos

$$
\left\{\begin{array}{l}
\frac{u_{j}^{k+1}-u_{j}^{k}}{\tau_{k}}=\sum_{i \in A_{j}} J\left(x_{j}-x_{i}\right) h_{i}\left(u_{i}^{k}-u_{j}^{k}\right), \quad k>0, \\
u_{j}^{0}=u_{0}\left(x_{j}\right)
\end{array}\right.
$$

onde $\tau_{k}=t_{k}-t_{k-1}$.

Denotamos por $U^{k+1}=\left(u_{1}^{k+1}, \cdots, u_{n+1}^{k+1}\right)$ o vetor cujas componentes satisfazem (4.8). Apresentamos então a definição de supersolução do problema (4.8).

Definição 4.1.2. Dizemos que $\bar{U}^{k+1}$ é uma supersolução de (4.8) se cada uma das suas componentes satisfaz

$$
\left\{\begin{array}{l}
\frac{\bar{u}_{j}^{k+1}-\bar{u}_{j}^{k}}{\tau_{k}} \geq \sum_{i \in A_{j}} J\left(x_{j}-x_{i}\right) h_{i}\left(\bar{u}_{i}^{k}-\bar{u}_{j}^{k}\right), \quad k>0, \\
\bar{u}_{j}^{0} \geq u_{0}\left(x_{j}\right) .
\end{array}\right.
$$

Analogamente, define-se $\underline{U}^{k+1}$ susbsolução de (4.8) invertendo as desigualdades.

Verificaremos então que vale um princípio de comparação para o esquema totalmente discreto.

Lema 4.1.3. Sejam $\bar{U}^{k+1}$ e $\underline{U}^{k+1}$ supersolução e subsolução do problema (4.8), respectivamente. Se $h$ é pequeno e o passo no tempo $\tau_{k}$ satisfaz

$$
\tau_{k}<\frac{1}{2}
$$

então $\underline{U}^{k} \leq \bar{U}^{k}, \forall k>0$.

Demonstração: Seja $Z^{k}=\bar{U}^{k}-\underline{U}^{k} \operatorname{com} z_{j}^{k}$ as componentes de $Z^{k}$. Como no Lema 4.1.1, podemos considerar desigualdades estritas, valendo então

$$
\left\{\begin{array}{l}
\frac{z_{j}^{k+1}-z_{j}^{k}}{\tau_{k}}>\sum_{i \in A_{j}} J\left(x_{j}-x_{i}\right) h_{i}\left[\left(\bar{u}_{i}^{k}-\bar{u}_{j}^{k}\right)-\left(\underline{u}_{i}^{k}-\underline{u}_{j}^{k}\right)\right], \\
z_{j}^{0}>0 .
\end{array}\right.
$$


Suponha que existe um nó $x_{j}$ e um primeiro tempo $t_{k+1}$ tal que $z_{j}^{k+1} \leq 0$. Daí temos

$$
\begin{aligned}
z_{j}^{k+1} & >z_{j}^{k}+\tau_{k} \sum_{i \in A_{j}} J\left(x_{j}-x_{i}\right) h_{i}\left(z_{i}^{k}-z_{j}^{k}\right) \\
& =z_{j}^{k}\left(1-\tau_{k} \sum_{i \in A_{j}} J\left(x_{j}-x_{i}\right) h_{i}\right)+\tau_{k} \sum_{i \in A_{j}} J\left(x_{j}-x_{i}\right) h_{i} z_{i}^{k} .
\end{aligned}
$$

Mas vale $\tau_{k} \sum_{i \in A_{j}} J\left(x_{j}-x_{i}\right) h_{i} z_{i}^{k}>0$ pois o primeiro tempo em que $z_{j} \leq 0$ é $t_{k+1}$. Logo

$$
\begin{aligned}
z_{j}^{k+1} & >z_{j}^{k}\left(1-\tau_{k} \sum_{i \in A_{j}} J\left(x_{j}-x_{i}\right) h_{i}\right) \\
& \geq z_{j}^{k}\left(1-\tau_{k}(1+O(h))\right),
\end{aligned}
$$

onde levamos em consideração a ordem da aproximação da integral, a ordem da partição e o valor de $\int_{\Omega} J(x) d x \leq 1$. Entretanto, $\tau_{k}<\frac{1}{2}$, então para $h$ suficientemente pequeno, temos que $\tau_{k}(1+O(h)) \leq$ $\frac{1}{2}$. Portanto, para tal $h, z_{j}^{k+1}>0$, absurdo. Logo, vale o princípio de comparação.

Observamos que a condição para o passo $\tau_{k}$ no caso geral (4.3) é

$$
\tau_{k}<\frac{1}{2(p-1) \max _{j}\left|\bar{u}_{j}^{k}\right|^{p-2}} .
$$

Pérez-Llanos e Rossi [PLR11] destacam que a condição para $\tau_{k}$ independe de $h$, diferentemente do que ocorre no problema local, cuja condição para o passo temporal é

$$
\tau_{k} \leq \frac{h^{p}}{2} .
$$

O resultado a seguir refere-se à convergência do método totalmente discreto.

Lema 4.1.4. Considere u uma solução positiva de (2.1) e $u_{h}^{k}$ dada por (4.8). Então existe $K$ tal que, para todo $h$ suficientemente pequeno, isto é, $0<h<h_{0}$ para algum $h_{0}$, vale

$$
\max _{0 \leq t_{k} \leq T}\left\{\max _{x_{j} \in[0, L]}\left|u\left(x_{j}, t_{k}\right)-\left(u_{h}^{k}\right)_{j}\right|\right\} \leq K(h+\tau)
$$

onde $h=\max h_{i}, \tau=\max \tau_{k}$ e $\left(u_{h}^{k}\right)_{j}$ é a j-ésima componente do vetor $u_{h}^{k}$.

Demonstração: Definimos o vetor erro no tempo $t_{k+1}$ como $\xi_{j}^{k+1}=u_{j}^{k+1}-v_{j}^{k+1}$, onde $v_{j}^{k+1}=$ $u\left(x_{j}, t_{k+1}\right)$. Vale então

$$
\begin{aligned}
\frac{\xi_{j}^{k+1}-\xi_{j}^{k}}{\tau_{k}} & =\sum_{i \in A_{j}} J\left(x_{j}-x_{i}\right) h_{i}\left(u_{i}^{k}-u_{j}^{k}\right)-\sum_{i \in A_{j}} J\left(x_{j}-x_{i}\right) h_{i}\left(v_{i}^{k}-v_{j}^{k}\right) \\
+ & \sum_{i \in A_{j}} J\left(x_{j}-x_{i}\right) h_{i}\left(v_{i}^{k}-v_{j}^{k}\right)-\frac{1}{\tau_{k}} \int_{t_{k}}^{t_{k+1}} \int_{\Omega} J\left(x_{j}-x_{i}\right)\left(u(y, s)-u\left(x_{j}, s\right)\right) d y d s \\
& \leq \sum_{i \in A_{j}} J\left(x_{j}-x_{i}\right)\left(\xi_{j}^{k}-\xi_{i}^{k}\right) h_{i}+C(h+\tau),
\end{aligned}
$$


pela aproximação de cada uma das integrais. De fato, definimos

$$
f_{i, k, j}(t)=\int_{t_{k}}^{t} \int_{\Omega} J\left(x_{j}-y\right)\left(u(y, s)-u\left(x_{j}, s\right)\right) d y d s .
$$

Expandindo em série de Taylor em torno de $t_{k}$, obtemos

$$
\frac{1}{\tau_{k}} f_{i, j, k}\left(t_{k+1}\right)=\frac{1}{\tau_{k}}\left(f_{i, j, k}\left(t_{k}\right)+\tau_{k} \int_{\Omega} J\left(x_{j}-y\right)\left(u\left(y, t_{k}\right)-u\left(x_{j}, t_{k}\right)\right) d y+C \tau_{k}^{2}\right),
$$

onde $C$ é uma constante que depende de $f$. Assim, vale

$$
\begin{aligned}
& \sum_{i \in A_{j}} J\left(x_{j}-x_{i}\right) h_{i}\left(v_{i}^{k}-v_{j}^{k}\right)-\frac{1}{\tau_{k}} \int_{t_{k}}^{t_{k+1}} \int_{\Omega} J\left(x_{j}-x_{i}\right)\left(u(y, s)-u\left(x_{j}, s\right)\right) d y d s \\
= & \sum_{i \in A_{j}} J\left(x_{j}-x_{i}\right) h_{i}\left(v_{i}^{k}-v_{j}^{k}\right)-\int_{\Omega} J\left(x_{j}-y\right)\left(u\left(y, t_{k}\right)-u\left(x_{j}, t_{k}\right)\right) d y+C \tau_{k} \\
\geq & O(h)+O(\tau) .
\end{aligned}
$$

Seja então $w_{j}^{k}=w^{k}=C t_{k}(h+\tau)$, para todo $j$ e todo $k$. Daí temos

$$
\begin{aligned}
\frac{w_{j}^{k+1}-w_{j}^{k}}{\tau_{k}} & =\frac{\left(C t_{k+1}-C t_{k}\right)(h+\tau)}{\tau_{k}} \\
& =\frac{C \tau_{k}(h+\tau)}{\tau_{k}} \\
& =C(h+\tau) \\
& \geq 0 .
\end{aligned}
$$

Observando que

$$
\sum_{i \in A_{j}} J\left(x_{j}-x_{i}\right)\left(w_{i}^{k}-w_{j}^{k}\right)=\sum_{i \in A_{j}} J\left(x_{j}-x_{i}\right)\left(C k t_{k}-C k t_{k}\right)(h+\tau)=0,
$$

concluímos que $w^{k}$ é supersolução do problema acima. Logo, $\xi_{j}^{k} \leq w^{k}=C t_{k}(h+\tau)$. Então tomando $K=C t_{k_{0}}$, obtemos a estimativa.

Concluímos então que o esquema totalmente discreto é convergente. Em [PLR11] são provadas outras propriedades dos esquemas semidiscreto e totalmente discreto, como preservação de massa e comportamento assintótico das soluções.

\subsection{Simulações numéricas}

As simulações numéricas que apresentamos a seguir foram realizadas utilizando a discretização total do problema. Destacamos que os resultados obtidos podem variar em decorrência de arredondamentos e da precisão com a qual os testes são feitos. Nas simulações utilizaremos a função densidade de probabilidade definida por

$$
J(s)=\left\{\begin{array}{l}
\frac{1}{2} \cos (s), \text { se }|s| \leq \pi / 2 \\
0, \text { caso contrário. }
\end{array}\right.
$$

Para a escolha da função $J$, buscamos uma função que satisfizesse todas as hipóteses mencionadas no Capítulo 2 e que tivesse suporte da forma $[-S, S]$, como exigido neste capítulo. Destacamos que $J$ não é $C^{1}$ em todo o domínio, mas satisfaz as outras hipóteses estabelecidas no Capítulo 3. 
O esquema utilizado no caso não linear é dado por (4.2) utilizando o método de Euler para obter a solução discreta do sistema de equações diferenciais ordinárias.

\subsubsection{Problema linear}

Primeiramente, analisamos três problemas lineares, que se referem ao Capítulo 2. Nos dois primeiros, utilizamos condições iniciais contínuas, enquanto no terceiro optamos por condição inicial $L^{\infty}(\Omega)$ constante por partes. 


\section{Problema 1}

Condição inicial: $u_{0}(x)=9-x^{2}$

Domínio: $\Omega=[-3,3]$

Valor médio da condição inicial: 6

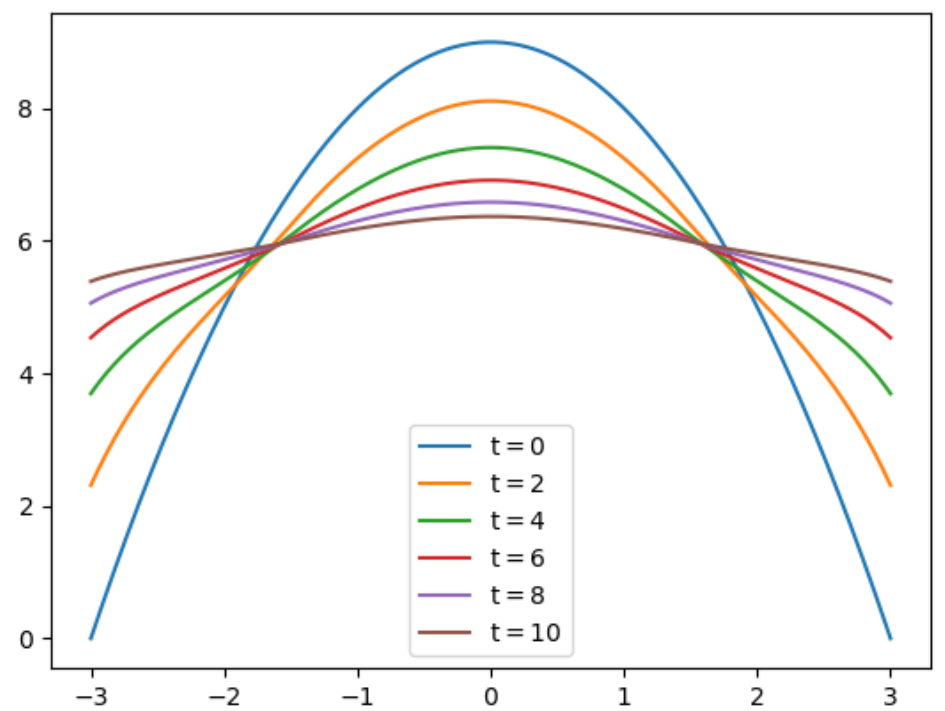

Figura 4.1: Gráfico da solução do problema 1 para determinados valores de $t$.

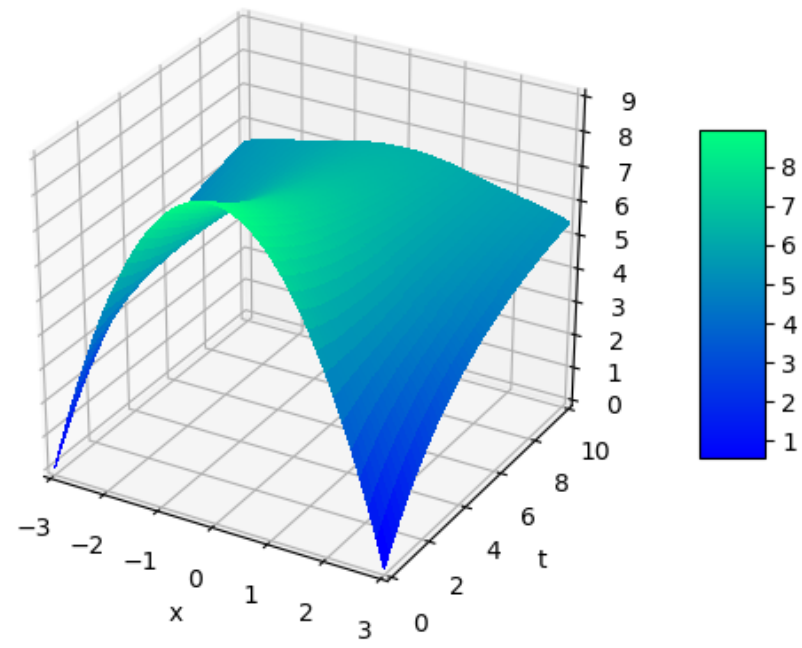

Figura 4.2: Gráfico da solução do problema 1.

\section{Problema 2}

Condição inicial: $u_{0}(x)=6 \cdot\left|\operatorname{sen} \frac{\pi x}{3}\right|+0,15(3-|x|)^{2}$

Domínio: $\Omega=[-3,3]$

Valor médio da condição inicial $\approx 4.27$. 


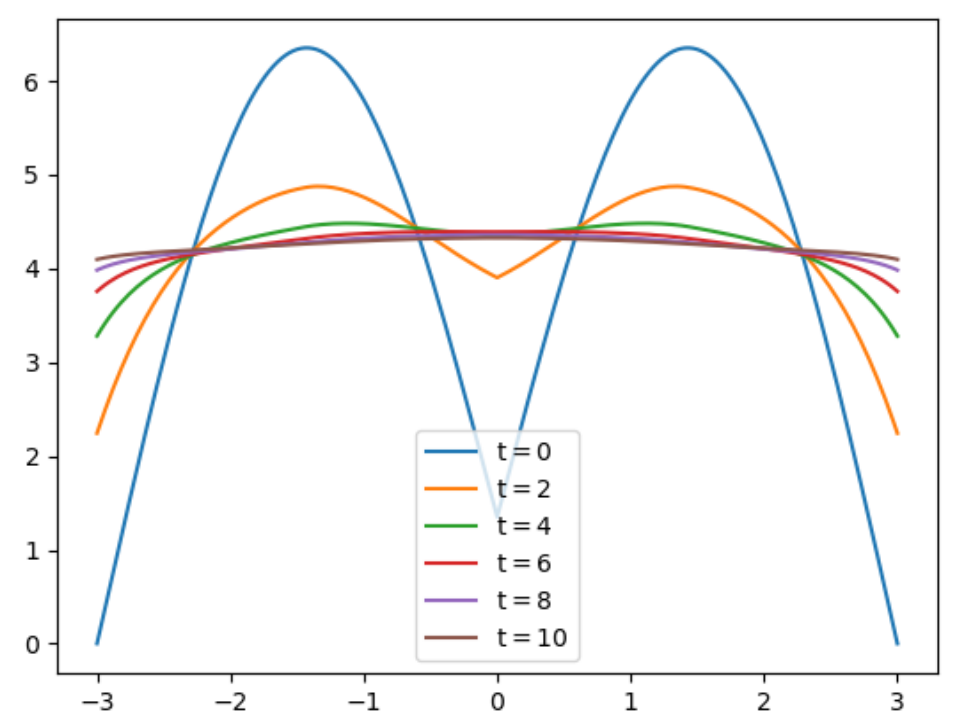

Figura 4.3: Gráfico da solução do problema 2 para determinados valores de $t$.

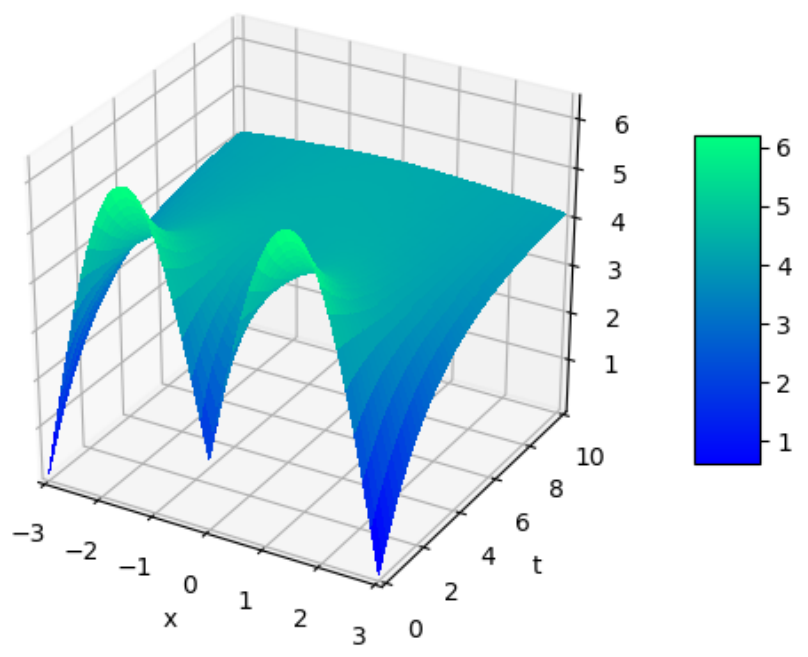

Figura 4.4: Gráfico da solução do problema 2.

\section{Problema 3}

Condição inicial: $u_{0}(x)=\left\{\begin{array}{l}1, \text { se } x \geq 0 \\ 0, \text { caso contrário. }\end{array}\right.$

Domínio: $\Omega=[-3,3]$

Valor médio da condição inicial: 0,5 . 


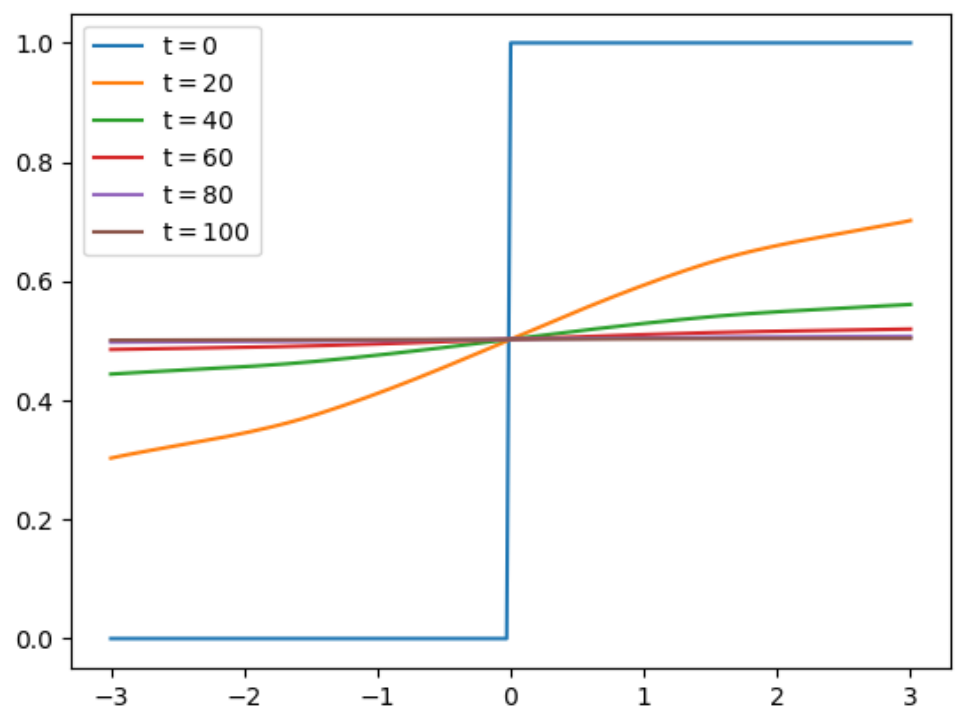

Figura 4.5: Gráfico da solução do problema 3 para determinados valores de $t$.

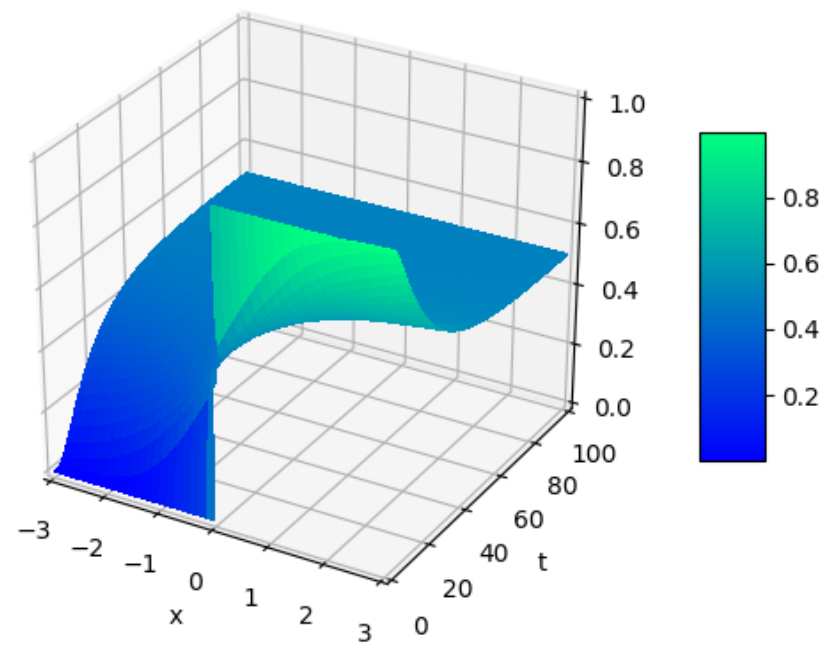

Figura 4.6: Gráfico da solução do problema 3.

Nas simulações acima observamos que as soluções tendem a uma constante no decorrer do tempo, relacionando-as ao Teorema 2.2.2. Esta constante é o valor médio da condição inicial. A convergência da solução contínua é garantida pelo teorema apresentado no Capítulo 2, mas PérezLlanos e Rossi apresentam em [PLR11] uma demonstração referente à convergência da solução do esquema totalmente discreto para o valor médio da condição inicial quando $t \rightarrow \infty$.

\subsubsection{Problema com termo de reação}

Os problemas apresentados a seguir ilustram alguns resultados discutidos no Capítulo 3. Os problemas 4 e 6 foram apresentados em [PLR09], porém com função $J$ descontínua, enquanto os 
demais apresentam alterações nas condições iniciais e foram realizados para observar como a solução se comporta de acordo com essas suposições.

Destacamos que as estimativas de tempo de explosão dependem da discretização utilizada no tempo. Utilizando um passo menor, podemos obter pequenas alterações nestas estimativas e uma aproximação melhor da solução, pois o esquema é convergente. Nas análises, estamos assumindo que a discretização utilizada preserva o tempo de explosão. O artigo [ALMM98] apresenta, para o caso local, uma discussão sobre a convergência do tempo de explosão do esquema semidiscreto em relação ao tempo de explosão da solução contínua. A discretização do termo não linear é a mesma utilizada em [PLR09]. Isto indica a possibilidade de que os argumentos utilizados para provar a convergência no caso local possam ser adaptados para o caso não local.

\section{Problema 4}

Condição inicial: $u_{0}(x)=9-x^{2}$

Tempo de explosão: $T \approx 6,19 \cdot 10^{-3}$

Domínio: $\Omega=[-3,3]$

Valor de $p: 3$.

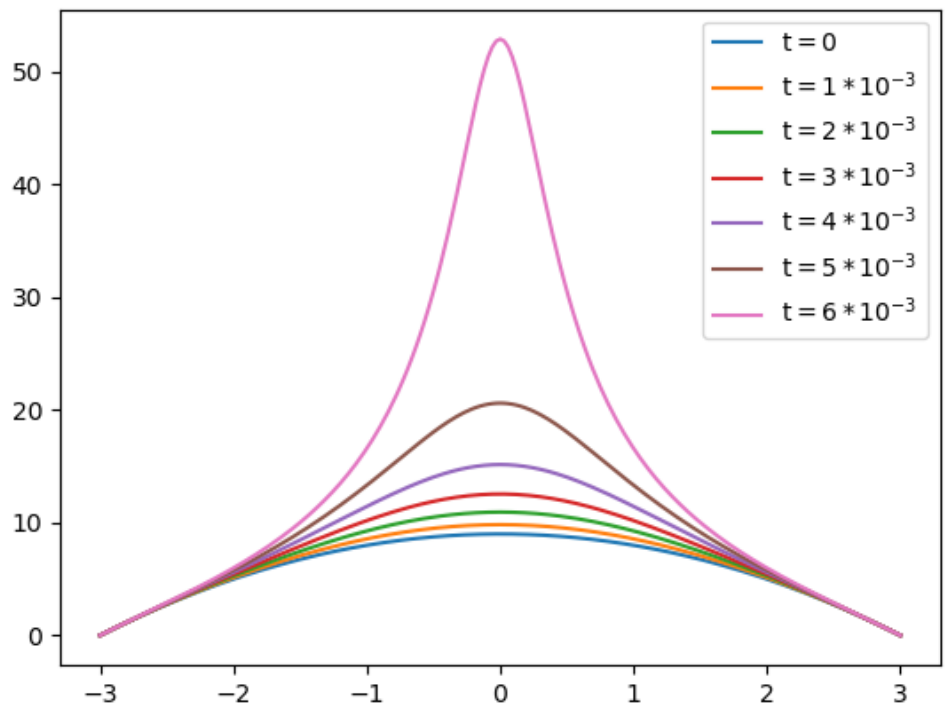

Figura 4.7: Gráfico da solução do problema 4 para determinados valores de $t$. 


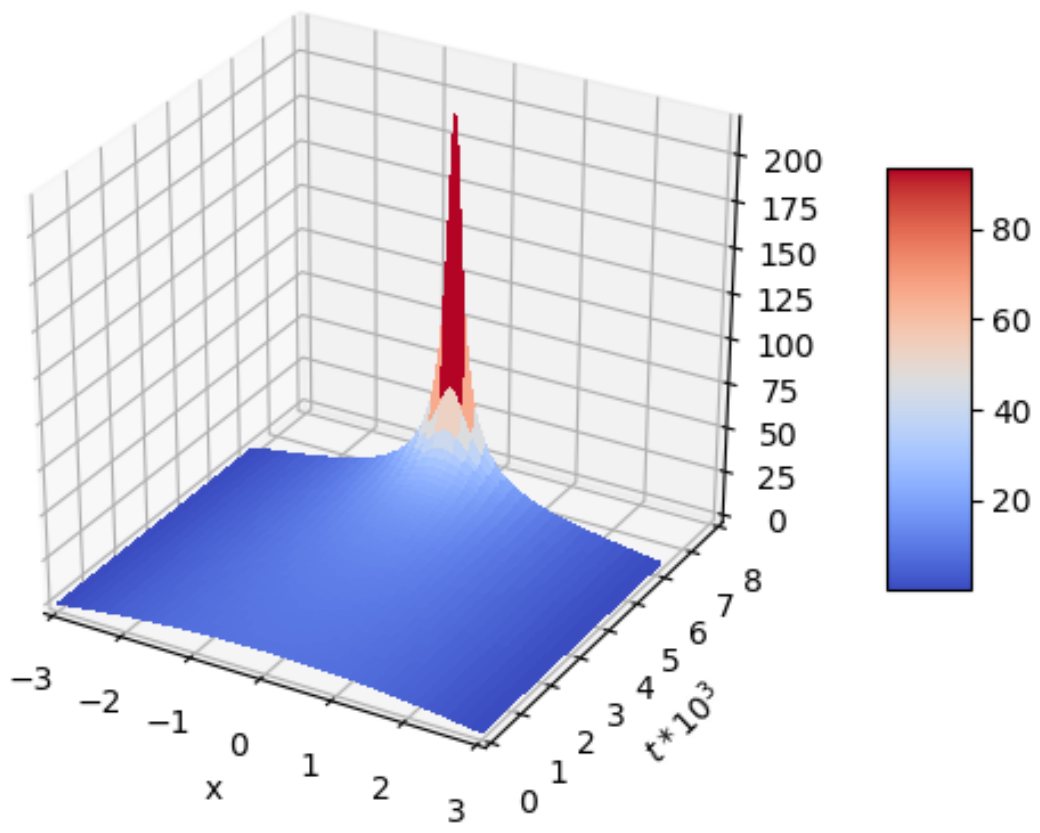

Figura 4.8: Gráfico da solução do problema 4.

Na figura (4.7) vemos que a condição inicial apresenta um máximo em $x=0$ e que a solução explode no mesmo valor, em conformidade com o resultado apresentado no Teorema (3.2.3), que discute o comportamento da solução no caso de condição inicial simétrica.

\section{Problema 5}

Condição inicial: $u_{0}(x)=9-x^{2}$

Tempo de explosão: $T \approx 4,585 \cdot 10^{-4}$

Domínio: $\Omega=[-3,3]$

Valor de $p: 4$ 


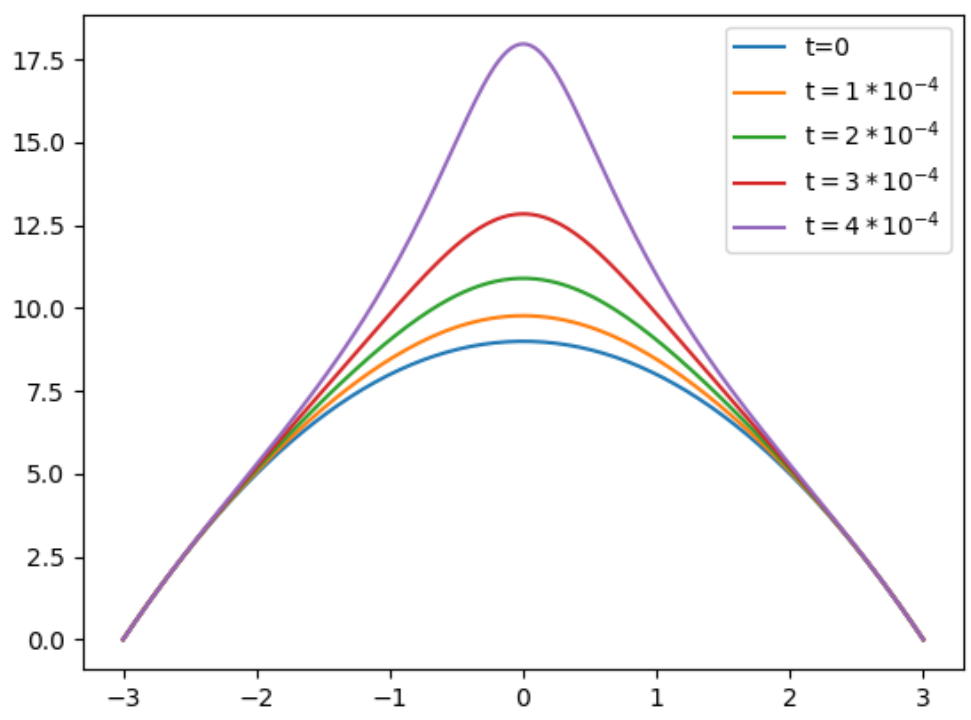

Figura 4.9: Gráfico da solução do problema 5 para determinados valores de $t$.

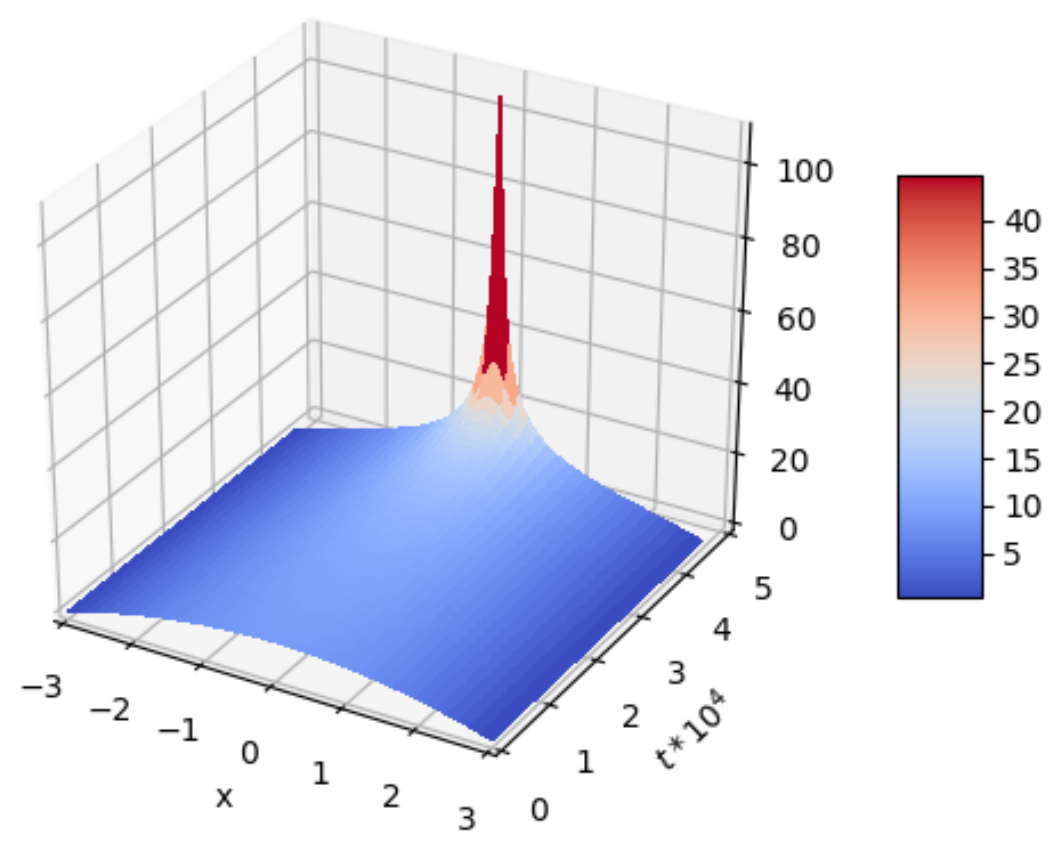

Figura 4.10: Gráfico da solução do problema 5.

Nesta simulação, alteramos o valor de $p$ e observamos que o tempo de explosão para este problema diminuiu. Este comportamento decorre do Teorema 3.2.2, que estabelece uma relação entre o valor de $p$ e a velocidade de crescimento da função. Assim, quanto maior o valor de $p$, mais rápido a solução explode.

Observamos também que o Teorema 3.2.1 indica uma estimativa superior para o valor de $T$. No 
caso do problema 4, o tempo de explosão deve ser menor que 0,0139, enquanto para o problema 5 , este deve ser menor do que 0,001544 . Isto se deve à relação entre o tamanho do domínio e a integral da condição inicial.

\section{Problema 6}

Condição inicial: $u_{0}(x)=\left\{\begin{array}{l}0,5+10(1-|1-x|), \text { se } 0 \leq x \leq 2 \\ 0,5, \text { caso contrário. }\end{array}\right.$

Tempo de explosão estimado: $T \approx 4,561 \cdot 10^{-3}$

Domínio: $\Omega=[-4,4]$

Valor de $p: 3$.

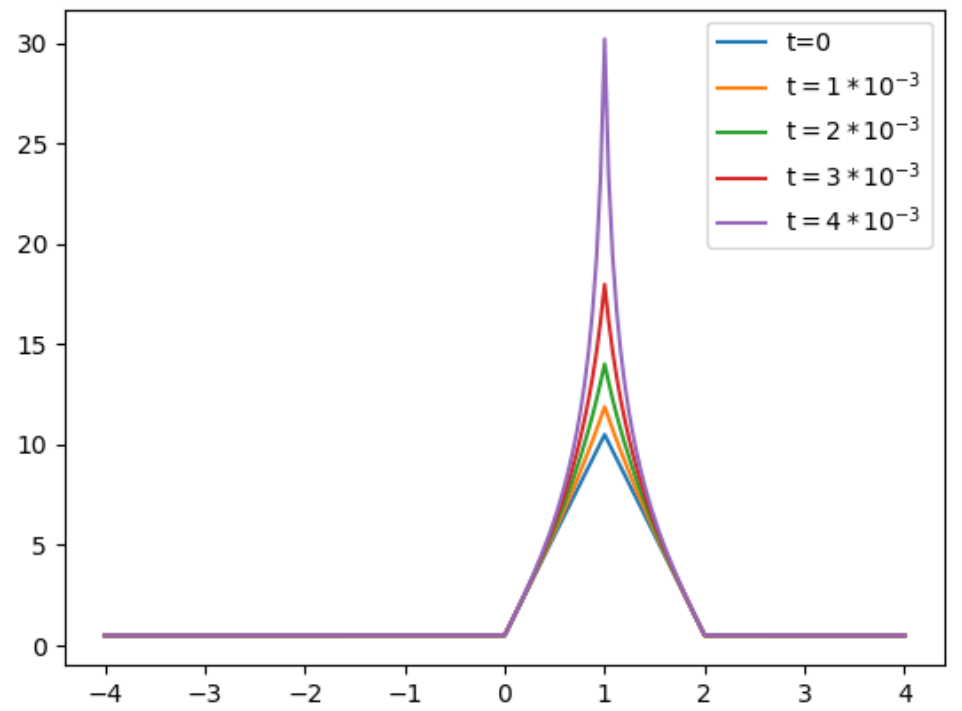

Figura 4.11: Gráfico da solução do problema 6 para determinados valores de $t$. 


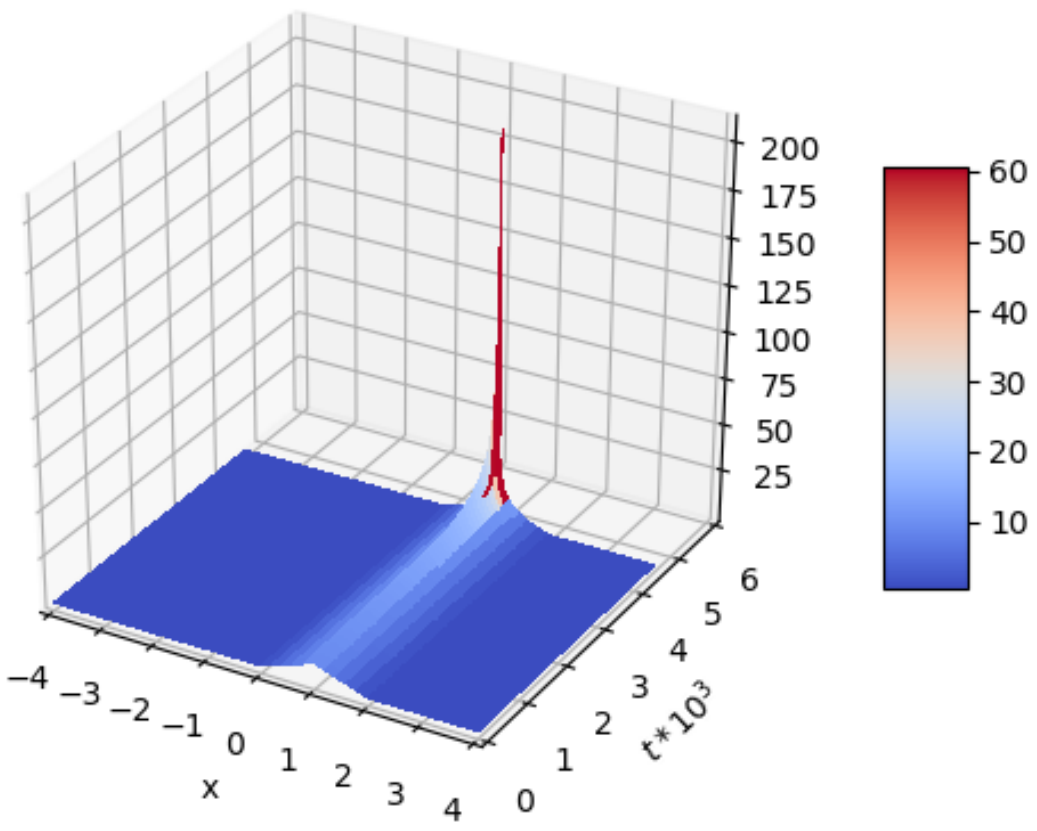

Figura 4.12: Gráfico da solução do problema 6 .

Nesta simulação, vemos um resultado que se relaciona com o Teorema 3.2.4. Observamos que a condição inicial possui um máximo em $x=1$ e assume valores altos na vizinhança deste ponto, enquanto se anula no restante do domínio. Assim, $u_{0}$ está dentro das condições para que a explosão ocorra em uma vizinhança de $x=1$, o que de fato ocorre, visto que a explosão é em $x=1$. Este exemplo indica que pode ser possível verificar um resultado similar ao Teorema 3.2.3 sem a necessidade de simetria.

\section{Problema 7}

Condição inicial: $u_{0}(x)=\cos (x)$

Tempo de explosão: $T \approx 5,778 \cdot 10^{-1}$

Domínio: $\left[-\frac{\pi}{2}, \frac{\pi}{2}\right]$

Valor de $p: 3$ 


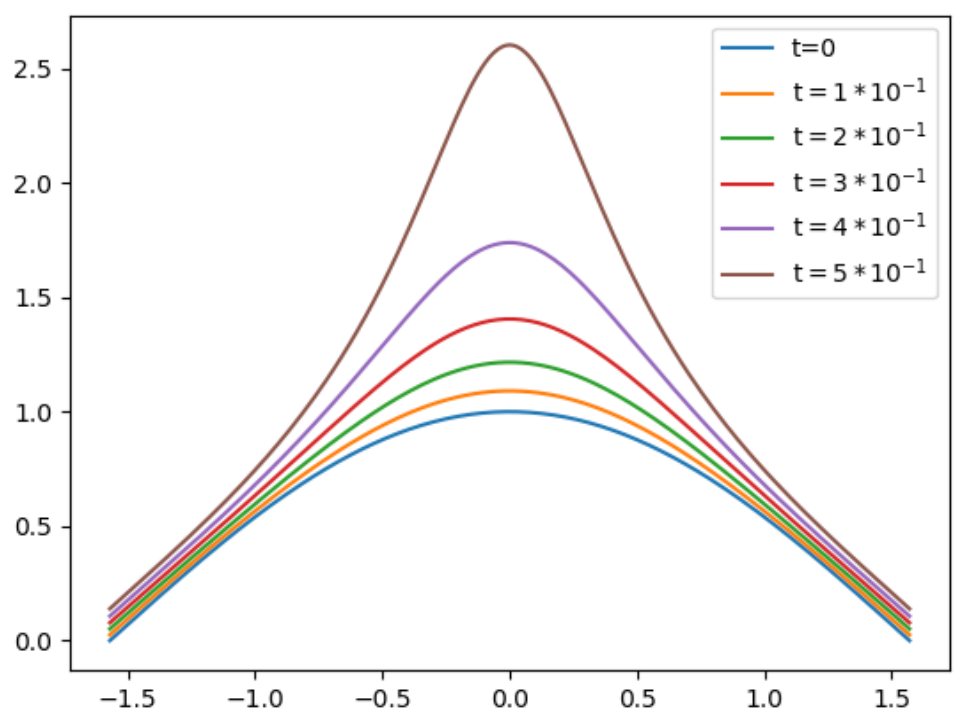

Figura 4.13: Gráfico da solução do problema 7 para determinados valores de $t$.

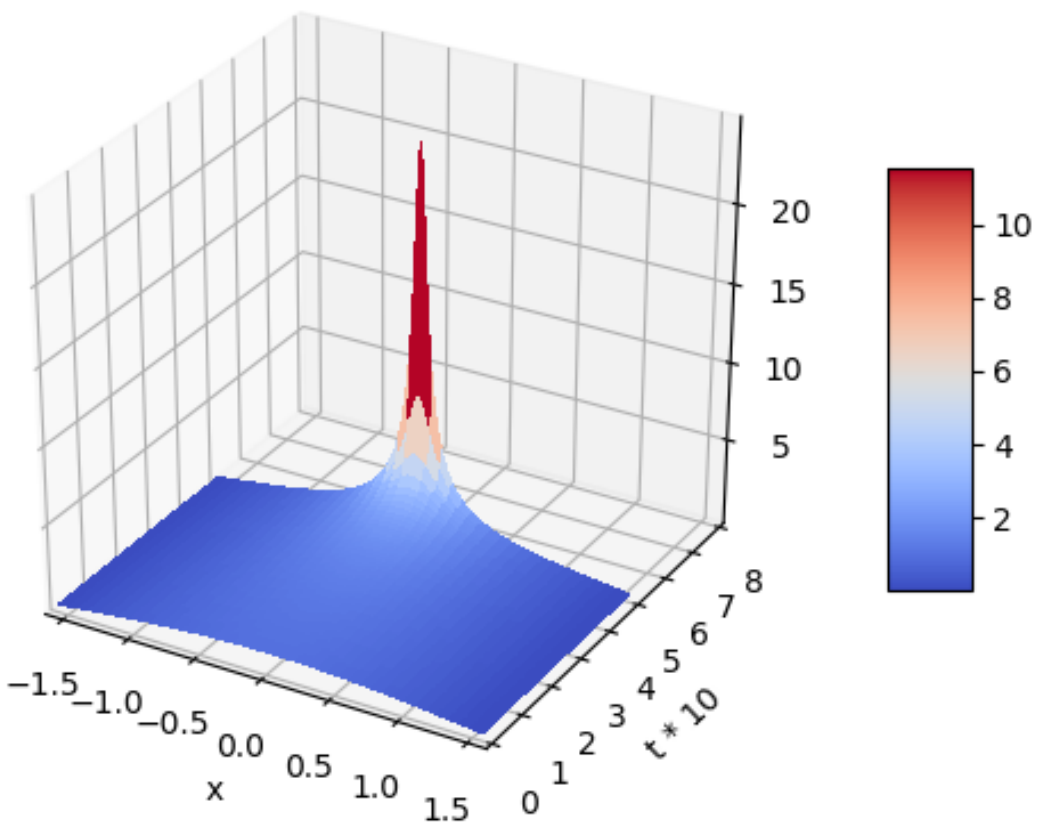

Figura 4.14: Gráfico da solução do problema $\%$.

A condição inicial deste problema é simétrica em relação a $x=0$ e apresenta comportamento semelhante ao primeiro exemplo. No entanto, a relação entre $|\Omega|$ e $\int_{\Omega} u_{0}(x) d x$ não é a mesma, pois neste caso, $\frac{|\Omega|}{\int_{\Omega} u_{0}(x) d x} \geq 1$. Isto indica que, ao aumentar o valor de $p$, estamos aumentando o limite superior de $T$, como ilustraremos no problema seguinte. 


\section{Problema 8}

Condição inicial: $u_{0}(x)=\cos (x)$

Tempo de explosão: $T \approx 3,346 \cdot 10^{-1}$

Domínio: $\left[-\frac{\pi}{2}, \frac{\pi}{2}\right]$

Valor de $p: 4$

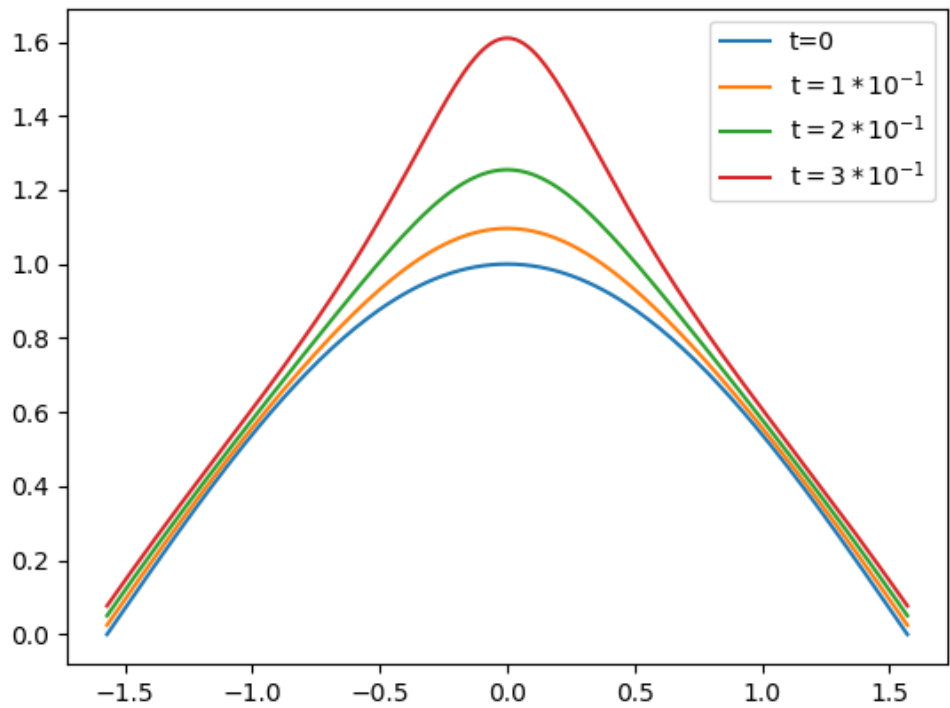

Figura 4.15: Gráfico da solução do problema 8 para determinados valores de $t$. 


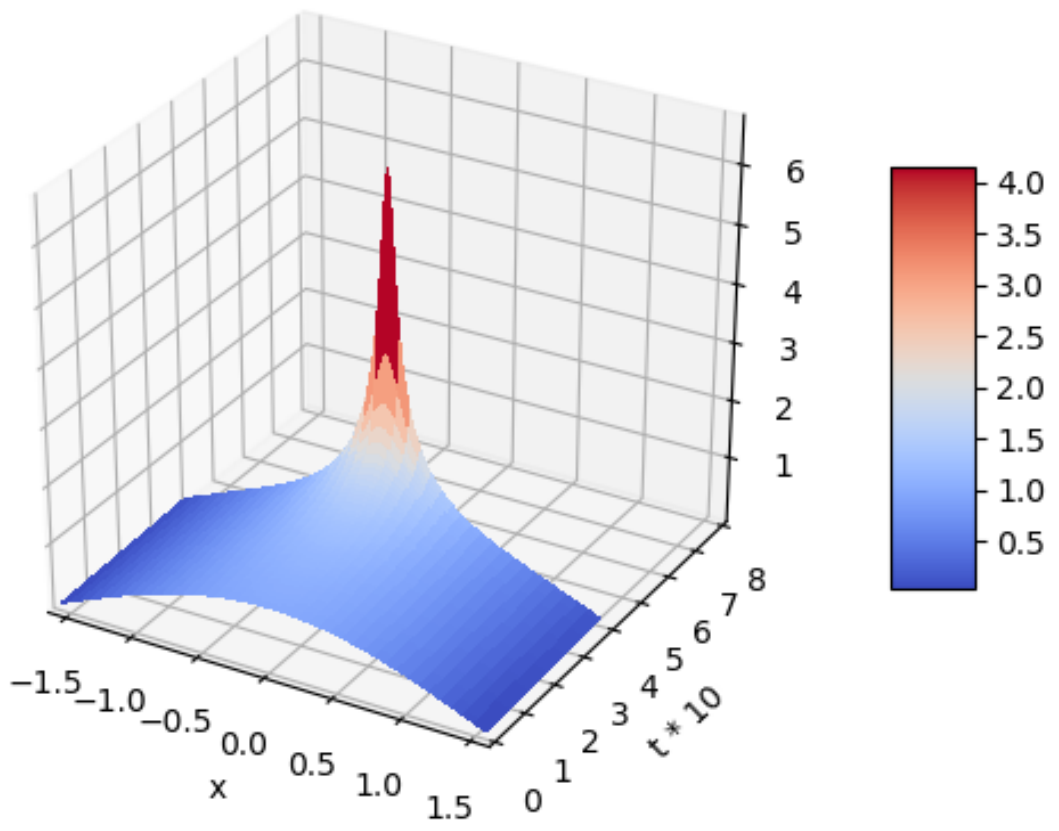

Figura 4.16: Gráfico da solução do problema 8.

Como vemos, o tempo de explosão do problema 8 é menor do que o encontrado para o problema 7, em decorrência da taxa de explosão estabelecida no Teorema 3.2.2. Porém, para o problema 7, a estimativa do tempo de explosão é $T \leq 1,2337$, enquanto para o problema 8 obtemos $T \leq 1,2919$. A estimativa superior cresce conforme aumentamos o valor de $p$, mas o tempo de explosão decresce. Assim vemos que mesmo se o limitante superior do tempo de explosão de um problema for mais alto do que de outro, isto não indica necessariamente que o tempo de explosão também será.

Por outro lado, a estimativa superior facilita a implementação do algoritmo de resolução do problema discretizado, reduzindo a quantidade de cálculos e tornando possível uma previsão da complexidade do algoritmo, além de indicar para quais valores de $p$ a solução explode.

\section{Problema 9}

Condição inicial:

$$
u_{0}(x)=\left\{\begin{array}{l}
\cos (x+0,5) \text { se } x<0 \\
\cos (x-0,5) \text { se } x \geq 0
\end{array}\right.
$$

Tempo de explosão: $T \approx 5,016 \cdot 10^{-1}$

Domínio: $\Omega=\left[-\frac{\pi}{2}, \frac{\pi}{2}\right]$

Valor de $p: 3$ 


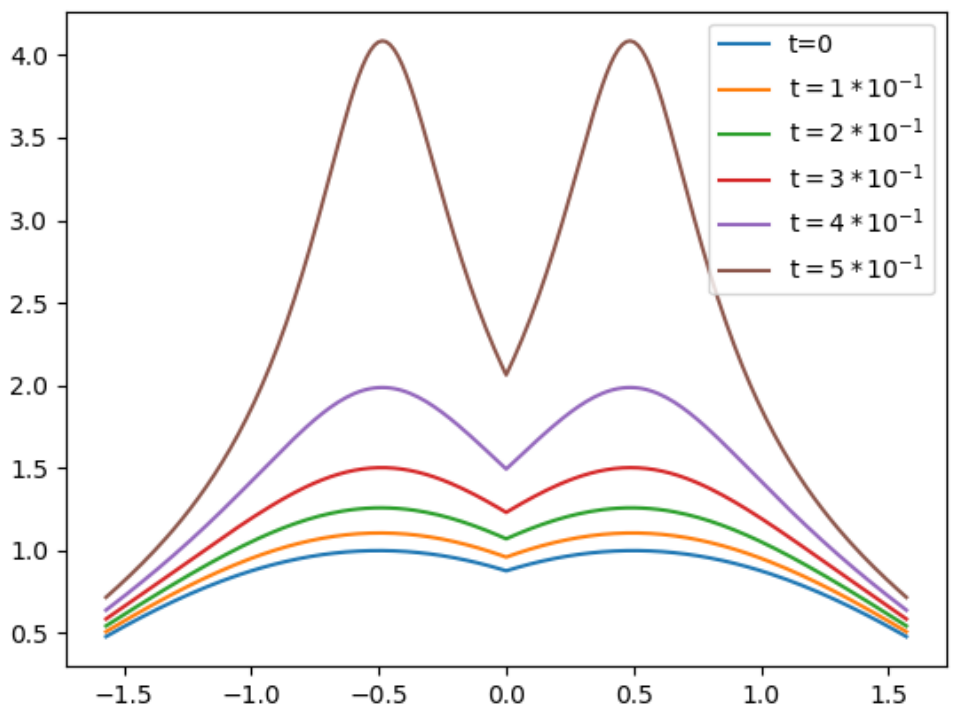

Figura 4.17: Gráfico da solução do problema 9 para determinados valores de $t$.

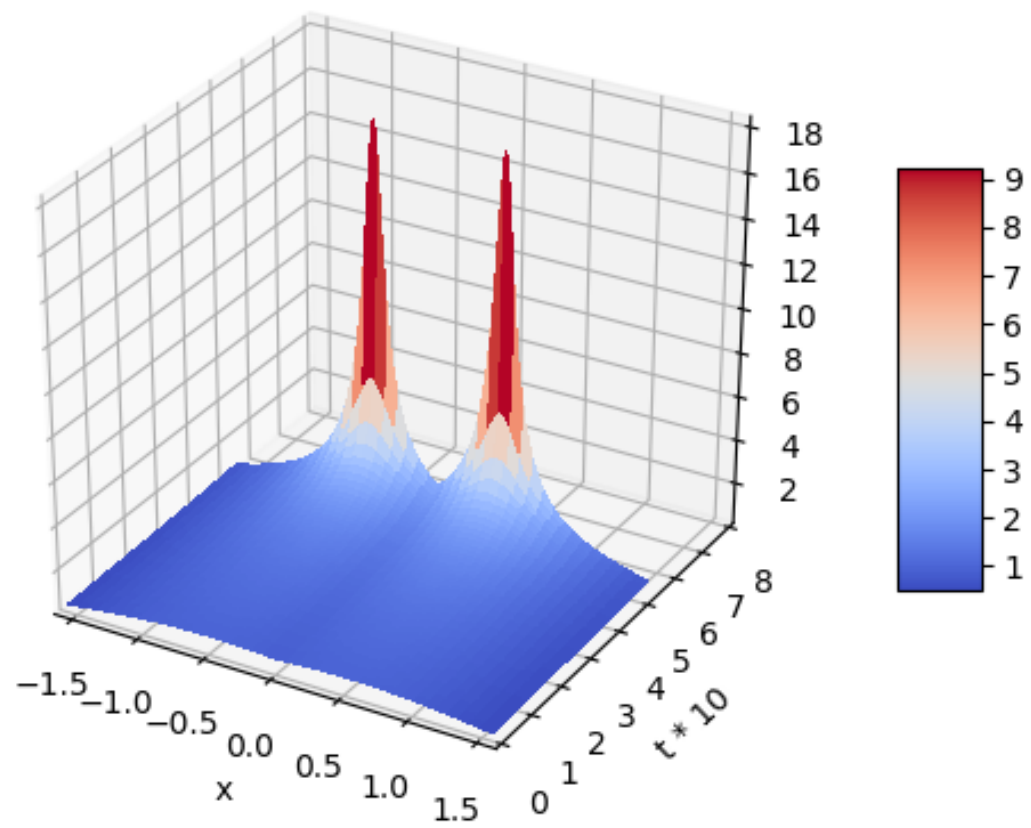

Figura 4.18: Gráfico da solução do problema 9.

Neste problema, utilizamos uma condição inicial simétrica com relação a $x=0$ e com dois máximos com distância de $\frac{1}{10}$. Verificamos que a solução apresenta dois pontos de explosão. Isto indica que pode ser possível demonstrar resultados semelhantes aos apresentados no Capítulo 3 para problemas mais gerais. No próximo problema, iremos diminuir a distância entre os pontos de máximo para verificar se este aspecto interfere comportamento dos pontos de explosão. 


\section{Problema 10}

Condição inicial:

$$
u_{0}(x)=\left\{\begin{array}{l}
\cos (x+0,01) \text { se } x<0 \\
\cos (x-0,01) \text { se } x \geq 0 .
\end{array}\right.
$$

Tempo de explosão: $T \approx 5,759 \cdot 10^{-1}$

Domínio: $\Omega=\left[-\frac{\pi}{2}, \frac{\pi}{2}\right]$

Valor de $p: 3$

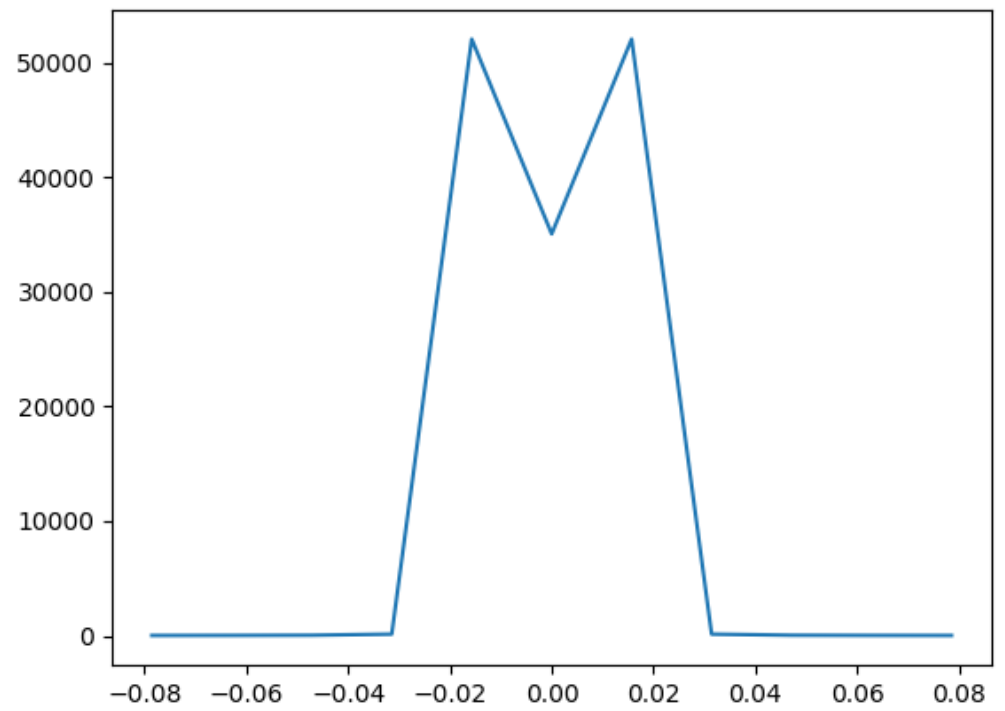

Figura 4.19: Gráfico da solução do problema 10 para $t=5,754 \cdot 10^{-1}$ no intervalo $[-0,08,0,08]$. 


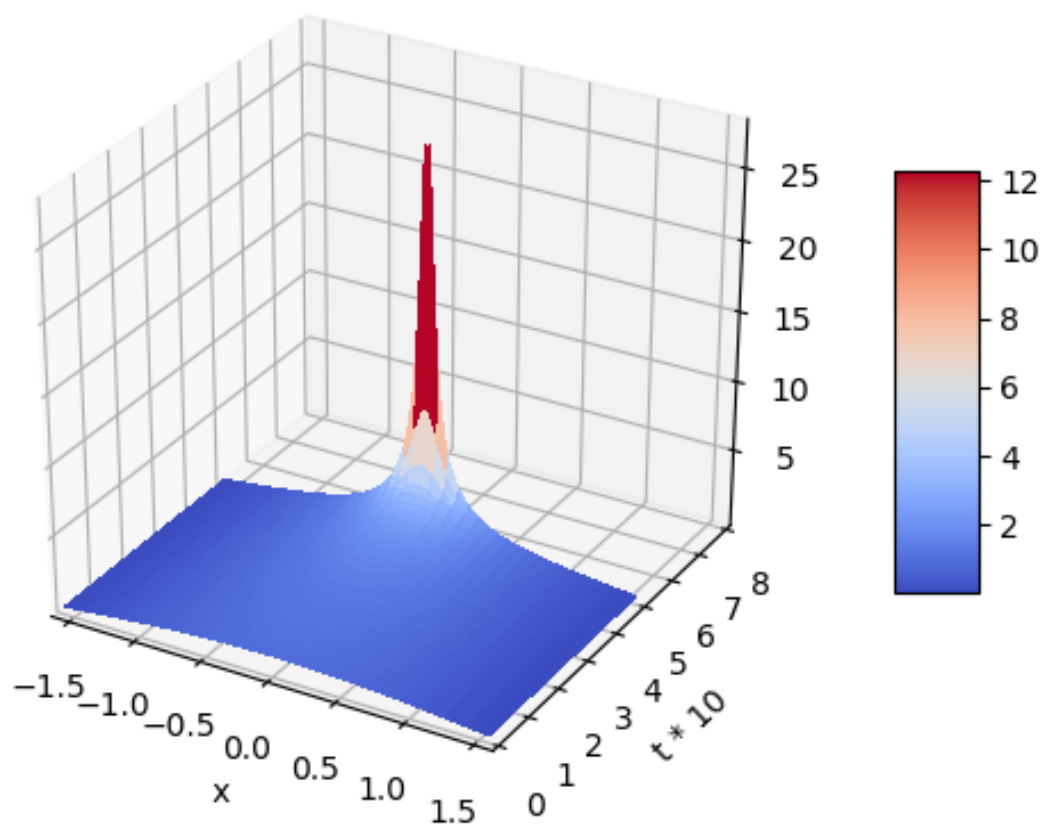

Figura 4.20: Gráfico da solução do problema 10.

Neste problema, aproximamos os pontos de máximo e verificamos que, apesar do crescimento da função em $x=0$ acompanhar o crescimento nos pontos onde a condição inicial atinge seu máximo, ainda obtivemos dois pontos de explosão simultâneos. Este resultado pode indicar que, mesmo nos casos em que a condição inicial possua mais de um ponto onde a função atinge seu máximo, o conjunto de pontos de explosão depende apenas destes pontos.

\section{Problema 11}

Condição inicial: $u_{0}(x)=\sin (x)+2$

Tempo de explosão: $T \approx 5,7 \cdot 10^{-2}$

Domínio: $\Omega=[-\pi, \pi]$

Valor de $p: 3$ 


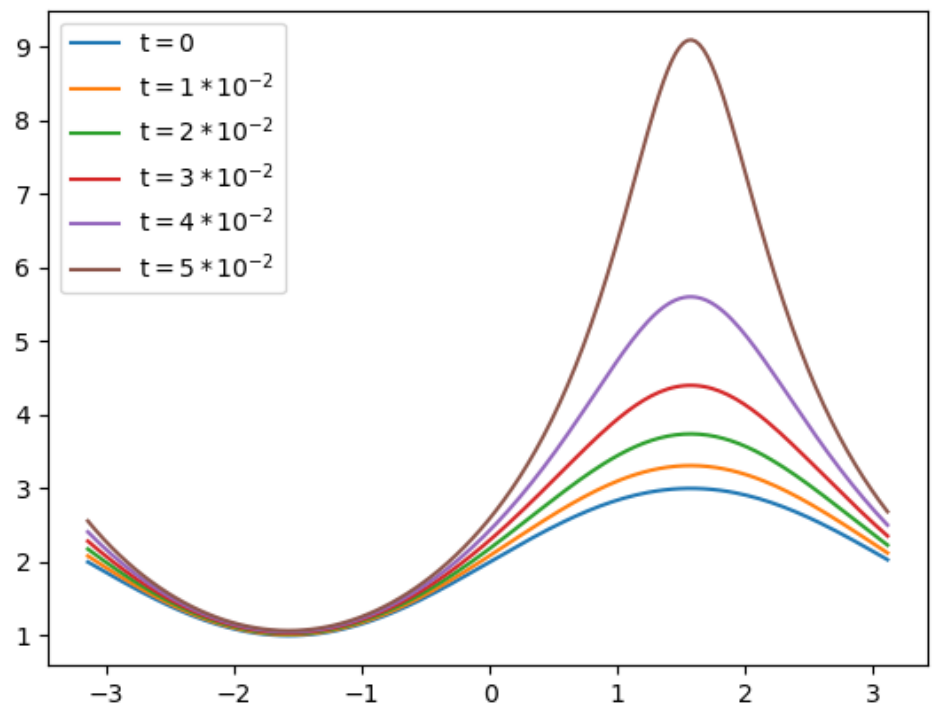

Figura 4.21: Gráfico da solução do problema 11 para determinados valores de $t$.

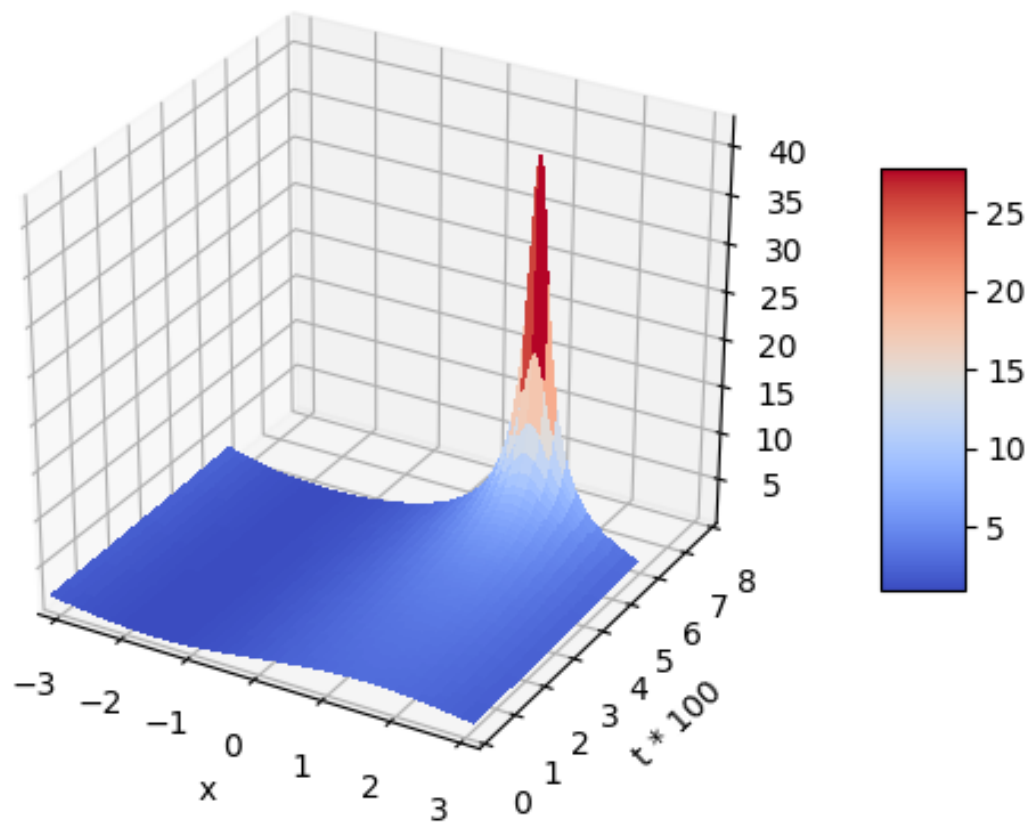

Figura 4.22: Gráfico da solução do problema 11.

Este problema o comportamento da solução com um ponto de máximo e um de mínimo. Vemos que, neste caso, a explosão da norma ocorre apenas no ponto de máximo, o que nos indica que o ponto de mínimo não interfere na posição do ponto de explosão. 


\section{Problema 12}

Condição inicial: $u_{0}(x)=\frac{1}{50}\left(-3 x^{4}+16 x^{3}-18 x^{2}+38\right)$

Tempo de explosão: $T \approx 3,168 \cdot 10^{-1}$

Domínio: $\Omega=[-1,4]$

Valor de $p: 3$

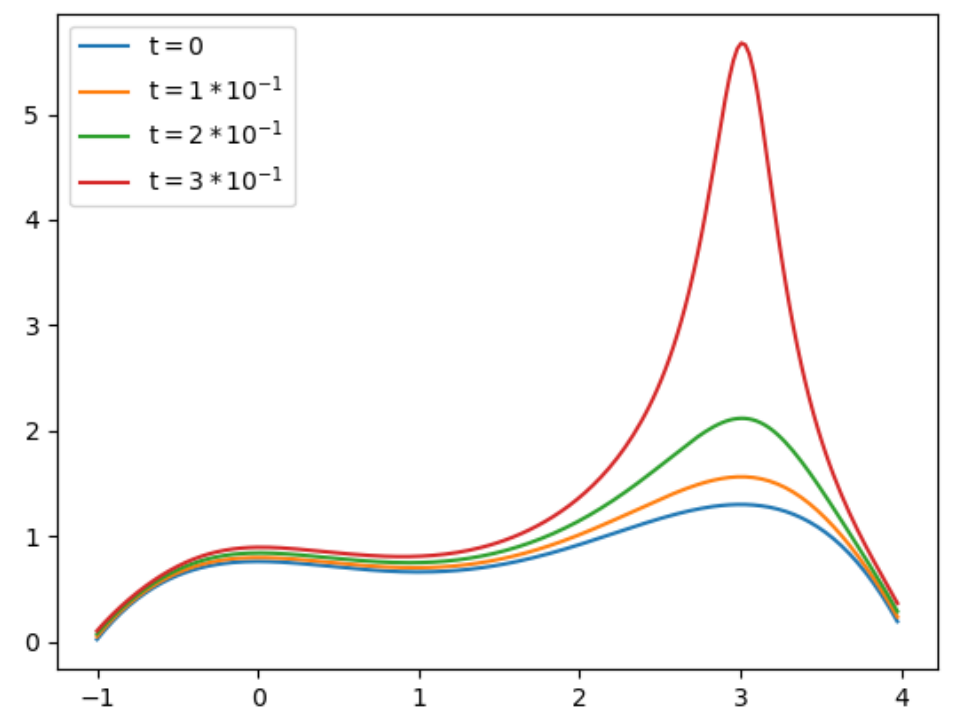

Figura 4.23: Gráfico da solução do problema 12 para determinados valores de $t$.

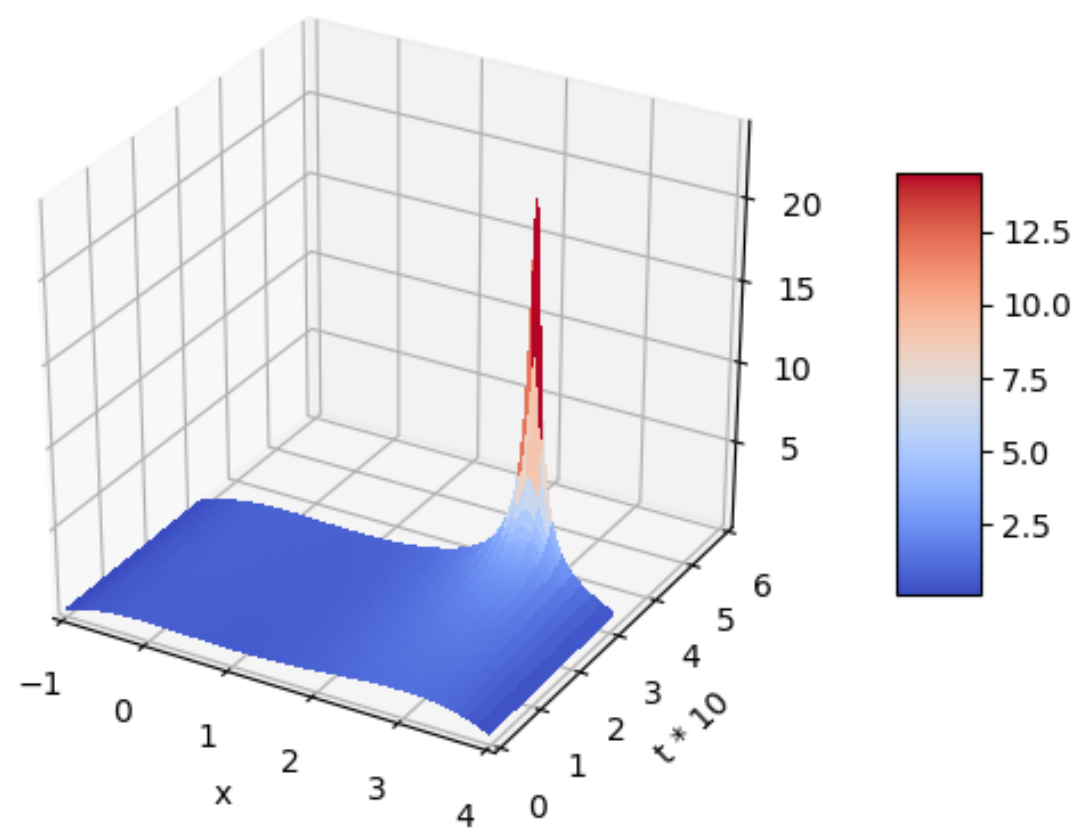

Figura 4.24: Gráfico da solução do problema 12. 
Neste problema, a condição inicial apresenta um ponto de máximo global e um local. Observamos que a explosão ocorre no ponto de máximo global, o que indica que o ponto de máximo local da condição inicial não interfere no ponto de explosão. 


\section{Conclusões}

Neste trabalho, apresentamos resultados referentes aos problemas de difusão não local mencionando algumas propriedades que são comuns ao problema local associado. Vimos que o problema do tipo Neumann não local linear pode ser entendido como a modelagem do comportamento de uma população em um habitat sem migração ou imigração. Formalmente analisamos a relação entre equações de reação-difusão regidas pelo Laplaciano apresentando uma versão não local, que aqui estudamos de maneira sistemática. Consideramos inicialmente a equação linear e posteriormente estudamos uma versão semilinear com não linearidades do tipo $u|u|^{p-1}$.

Em relação ao problema linear, observamos que podemos garantir existência e unicidade de soluções desde que tomemos uma condição inicial $u_{0} \in L^{1}(\Omega)$. Vimos também que esta solução apresenta comportamento assintótico similar ao Laplaciano com condições de contorno de Neumann homogêneas convergindo para o valor médio da condição inicial. Além disso, obedece o princípio de comparação para soluções contínuas. No caso do problema não linear com não linearidade $u|u|^{p-1}$, vimos que podemos garantir existência e unicidade de soluções para tempo finito se $p>1$ e para tempo infinito se $p \leq 1$. Além disso, podemos estabelecer estimativas superiores para o tempo de explosão e resultados referentes ao ponto de explosão.

Estudando e utilizando a discretização do problema linear, pudemos elaborar simulações referentes aos problemas estudados. Analisando os gráficos obtidos por meio da discretização dos problemas, foi possível observar os resultados apresentados e conjecturar sobre outras possibilidades de estudo rigoroso.

Como sugestões para pesquisas futuras, destacamos a possibilidade de adaptar os argumentos apresentados para cenários mais amplos. Por exemplo, demonstrar propriedades referentes aos problemas de explosão com condições iniciais não simétricas ou que atingem o valor máximo em mais de um ponto. Além disso, os resultados aqui apresentados podem ser utilizados como base para estudo de outras não linearidades, que são aplicáveis a outras áreas. 


\section{Referências Bibliográficas}

[ALMM98] Luis M Abia, JC López-Marcos e Julia Martínez. On the blow-up time convergence of semidiscretizations of reaction-diffusion equations. Applied numerical mathematics, 26(4):399-414, 1998. 53

[AVt08] Fuensanta Andreu-Vaillo et al. A nonlocal p-laplacian evolution equation with neumann boundary conditions. Journal de mathématiques pures et appliquées, 90(2):201-227, 2008. 42

[AVt10] Fuensanta Andreu-Vaillo et al. Nonlocal diffusion problems. Number 165. American Mathematical Soc., 2010. 1, 5, 9, 19

[BC02] Peter W Bates e Fengxin Chen. Spectral analysis and multidimensional stability of traveling waves for nonlocal allen-cahn equation. Journal of mathematical analysis and applications, 273(1):45-57, 2002. 3

[Bre10] Haim Brezis. Functional analysis, Sobolev spaces and partial differential equations. Springer Science \& Business Media, 2010. 14

[CF05] Claudia Carrillo e Paul Fife. Spatial effects in discrete generation population models. Journal of Mathematical Biology, 50(2):161-188, 2005. 3

[Fif03] Paul Fife. Some nonclassical trends in parabolic and parabolic-like evolutions. Em Trends in nonlinear analysis, páginas 153-191. Springer, 2003. 1, 5

[FL06] Nicolas Fournier e Philippe Laurençot. Well-posedness of smoluchowski's coagulation equation for a class of homogeneous kernels. Journal of functional Analysis, 233(2):351379, 2006. 3

[FPL12] Raúl Ferreira e Mayte Pérez-Llanos. Blow-up for the non-local p-laplacian equation with a reaction term. Nonlinear Analysis: Theory, Methods \& Applications, 75(14):5499-5522, 2012. 31

[GO07] Guy Gilboa e Stanley Osher. Nonlocal linear image regularization and supervised segmentation. Multiscale Modeling ES Simulation, 6(2):595-630, 2007. 1

[GO08] Guy Gilboa e Stanley Osher. Nonlocal operators with applications to image processing. Multiscale Modeling ES Simulation, 7(3):1005-1028, 2008. 3

[Góm14] Silvia Sastre Gómez. Nonlocal diffusion problems. Tese de Doutorado, Universidad Complutense de Madrid, 2014. 1, 3, 5, 21, 25, 26

[GR01] Pablo Groisman e Julio D Rossi. Asymptotic behaviour for a numerical approximation of a parabolic problem with blowing up solutions. Journal of computational and applied mathematics, 135(1):135-155, 2001. 1, 33, 38

[HMMV03] Vivian Hutson, Salome Martinez, Konstantin Mischaikow e Glenn T Vickers. The evolution of dispersal. Journal of mathematical biology, 47(6):483-517, 2003. 3 
[Hön61] Chaim Samuel Hönig. Aplicações da topologia à análise, volume 8. Instituto de Física e Matemática, Universidade do Recife, 1961. 24, 25

[KA06] P. Knabner e L. Angerman. Numerical Methods for Elliptic and Parabolic Partial Differential Equations. Texts in Applied Mathematics. Springer New York, 2006. 42

[OS17] Christian Olivera e Marielle Simon. Non-local conservation law from stochastic particle systems. arXiv preprint arXiv:1701.0467r, 2017. 1

[PLR09] Mayte Pérez-Llanos e Julio D Rossi. Blow-up for a non-local diffusion problem with neumann boundary conditions and a reaction term. Nonlinear Analysis: Theory, Methods E Applications, 70(4):1629-1640, 2009. 1, 10, 21, 27, 29, 31, 33, 38, 41, 52, 53

[PLR11] Mayte Pérez-Llanos e Julio D Rossi. Numerical approximations for a nonlocal evolution equation. SIAM Journal on Numerical Analysis, 49(5):2103-2123, 2011. 1, 41, 47, 48, 52

[SMD18] Richard Mikaël Slevinsky, Hadrien Montanelli e Qiang Du. A spectral method for nonlocal diffusion operators on the sphere. Journal of Computational Physics, 2018. 3

[TD13] Xiaochuan Tian e Qiand Du. Analysis and comparison of different approximations to nonlocal diffusion and linear peridynamic equations. SIAM Journal on Numerical Analysis, 51(6):3458-3482, 2013. 1

[Tt18] Yunzhe Tao et al. Nonlocal neural networks, nonlocal diffusion and nonlocal modeling. arXiv preprint arXiv:1806.00681, 2018. 3 